\title{
Propriétés optiques des semi-conducteurs et de leurs hétérostructures
}

\author{
R. Planel \\ Laboratoire de Microstructures et de Microélectronique (L2M), UPR-CNRS, BP. 107, \\ 92225 Bagneux cedex, France
}

\begin{abstract}
Résumé : Je pense, dans ce chapitre, présenter l'essentiel de ce qu'un opticien doit savoir pour utiliser à bon escient les « matériaux » semi-conducteurs, avant d'approfondir son propre sujet. Il est donc d'abord question des propriétés optiques linéaires, secondairement de diverses causes possibles de non-linéarités. Enfin, je n'oublie pas qu'aujourd'hui, chacun doit avoir présent à l'esprit la possibilité de réaliser, souvent à la demande, des hétérostructures de basse dimensionnalité. Les plus courantes aujourd'hui restent les structures bidimensionnelles, que je présente plus en détail. Beaucoup sont facilement réalisables ; de plus elles représentent encore l'optimum de ce qu'il est possible de faire en matière de "génie des hétérostructures ». Les structures à zéro dimension font l'objet, sur un exemple particulier, d'un chapitre différent dans ce livre.
\end{abstract}

\section{INTRODUCTION}

Les semi-conducteurs ne sont pas avant tout des matériaux pour l'optique. On les définit d'abord, dans le vaste domaine de la physique des solides, par leur conductivité, intermédiaire entre celle des métaux et des isolants; et surtout par sa dépendance en température, la conductivité étant thermiquement activée. Au plan des applications, c'est d'abord d'électronique qu'il s'agit. On pourra, pour s'en convaincre, compter le nombre de pages consacrées aux propriétés optiques des semi-conducteurs dans des livres de physique des solides classiques de niveau $3^{\text {ieme }}$ cycle (Kittel [1], Ashcroft-Mermin [2]), ou même dans un livre plus centré sur la physique des semi-conducteurs et des composants (Mathieu [3]).

Cependant, ces livres sont très recommandables à ceux qui souhaiteront approfondir ce chapitre introductif dans les domaines de la théorie des bandes et des propriétés électriques. Celui d'Henry Mathieu va beaucoup plus loin que les deux autres dans le domaine des composants (il est, de plus, écrit en français). Ces dernières années ont cependant vu apparaître plusieurs cours où les propriétés optiques des semi-conducteurs sont beaucoup mieux traités (Haug et Koch [4], Klingshirn [5], Yu et Cardona [6]). Je recommande en particulier ce dernier. On pourra d'abord y combler des lacunes de ce cours-ci, par exemple sur les phonons. De plus afin de pouvoir ici aller à l'essentiel, j'ai choisi d'y renvoyer le lecteur plus curieux, pour des calculs plus rigoureux ou des situations plus complexes, sous la forme suivante : [YC p. ...] ; dans la mesure du possible, j'ai essayé d'utiliser les mêmes notations. Le livre de Klingshirn est plus expérimental, plus centré sur l'optique, mais plus qualitatif. Celui 
de Haug et Koch est beaucoup moins complet, mais il sera très utile à qui veut établir les liens théoriques avec l'optique non linéaire classique.

Dans ce cours en français, je n'ai pas cherché à citer systématiquement les premiers auteurs de travaux classiques comme il aurait convenu dans un article de revue. De plus, il faut être conscient de la concurrence internationale aiguë qui marque ces sujets, avec de très nombreux doublons et une surpublication dramatique. Une véritable revue raisonnée, citant tout ce qui est digne d'intérêt sur ce que j'aborde dans ce cours compterait probablement plusieurs centaines de publications, avec beaucoup de travaux américains, japonais et, en Europe, allemands. Face à ce problème, j'ai choisi de citer en priorité les travaux français (et proches) lorsqu'ils étaient au niveau de qualité international. De plus je leur ai volontiers emprunté des figures et des résultats. C'était plus commode pour moi, mais cela présente aussi l'intérêt de favoriser les prises de contact, voire les collaborations, pour les lecteurs qui voudraient aller plus loin. Dans cet esprit, je dois faire un peu de publicité pour les actes du Symposium Claude Benoit à la Guillaume [7], où une bonne part de la communauté française des opticiens des semi-conducteurs et quelques collègues étrangers proches se sont réunis en hommage à ce pionnier. À cette occasion, de nombreux articles de synthèse ont été écrits avec plus de recul que les publications primaires.

Venons-en maintenant au sujet. Dans l'enseignement académique, on se place assez vite dans le cadre de la théorie des bandes, associée à l'état cristallin, pour dire qu'un semiconducteur est un isolant à faible bande interdite (gap); avec "faible ", on précise généralement quelques kT (énergie thermique à la température ambiante). Mais en réalité, les matériaux étant rarement très purs, c'est surtout à l'énergie de liaison des porteurs de charge sur les impuretés, donneurs ou accepteurs, qu'il faut s'intéresser. Et le critère d'un " bon " semi-conducteur serait plutôt que les états électroniques des bandes sont " bien " délocalisés sur de très nombreux sites, avec une largeur de bande importante et donc une masse effective faible, de l'ordre de ou inférieure à celle de l'électron libre. Dans la suite, nous pourrons donner un contenu plus objectif à ces notions. Mais il en résulte que les états électroniques conduiraient bien s'ils étaient effectivement peuplés d'électrons (dans la bande de conduction) ou de trous (dans la bande de valence). Tel est, je pense, le critère essentiel pour un semiconducteur. Et alors, le diamant avec son gap supérieur à $5 \mathrm{eV}$, apparaît un bon semiconducteur, comme, naturellement InSb (gap $0.17 \mathrm{eV}$ ) ou GaAs (gap $1.4 \mathrm{eV}$ ). Dans ces conditions, l'intérêt premier des semi-conducteurs est dans la possibilité de moduler grandement la densité de ces porteurs (de charge), en jouant sur la température, mais surtout sur le dopage (concentration d'impuretés électriquement actives), ou encore, dans un dispositif, par un champ électrique appliqué ou une illumination. De cette faculté découle l'importance de l'industrie de la microélectronique, avec ses caractéristiques qui donnent le tournis : un marché de centaines de milliards de dollars, la miniaturisation des circuits par l'intégration de millions de transistors par $\mathrm{cm}^{2}$, le pas élémentaire du dessin des circuits qui a franchi, il y a déjà plusieurs années, la barre symbolique du micron. De plus toutes ces évolutions se font au rythme d'une loi exponentielle empirique, énoncée par Moore il y a trente ans, et qui depuis se vérifie sous les yeux ébahis de l'ensemble des spectateurs, et des acteurs eux-mêmes (Cf. fig.1.1). Et cette industrie aux retombées sans équivalent dans l'histoire a aussi ses faiblesses : le gigantisme des investissements et l'inertie de la fabrication, qui rendent difficile d'y introduire des innovations ne s'inscrivant pas dans les voies prévues.

Cependant, les progrès de la technologie, stimulés par cette course à l'intégration et à la miniaturisation, ont eu aussi des retombées dans des domaines plus éloignés des applications, voire fondamentaux. Ce furent les premières réalisations d'hétérostructures dans les années 1970, et, aujourd'hui, la maturité impressionnante de certaines techniques (l'épitaxie par 
exemple), l'audace de certaines autres (les techniques de champ proche). Le nombre de communications consacrées aux hétérostructures à la Conférence Internationale sur la Physique des Semi-Conducteurs a cru exponentiellement de moins de 5 jusqu'en 1978, à 200 vers 1988 , représentant environ $50 \%$ des contributions. Depuis, ce pourcentage a tendance à saturer. À celle de Montpellier, en 1982, Pierre Aigrain a parlé d'une nouvelle jeunesse pour

a)

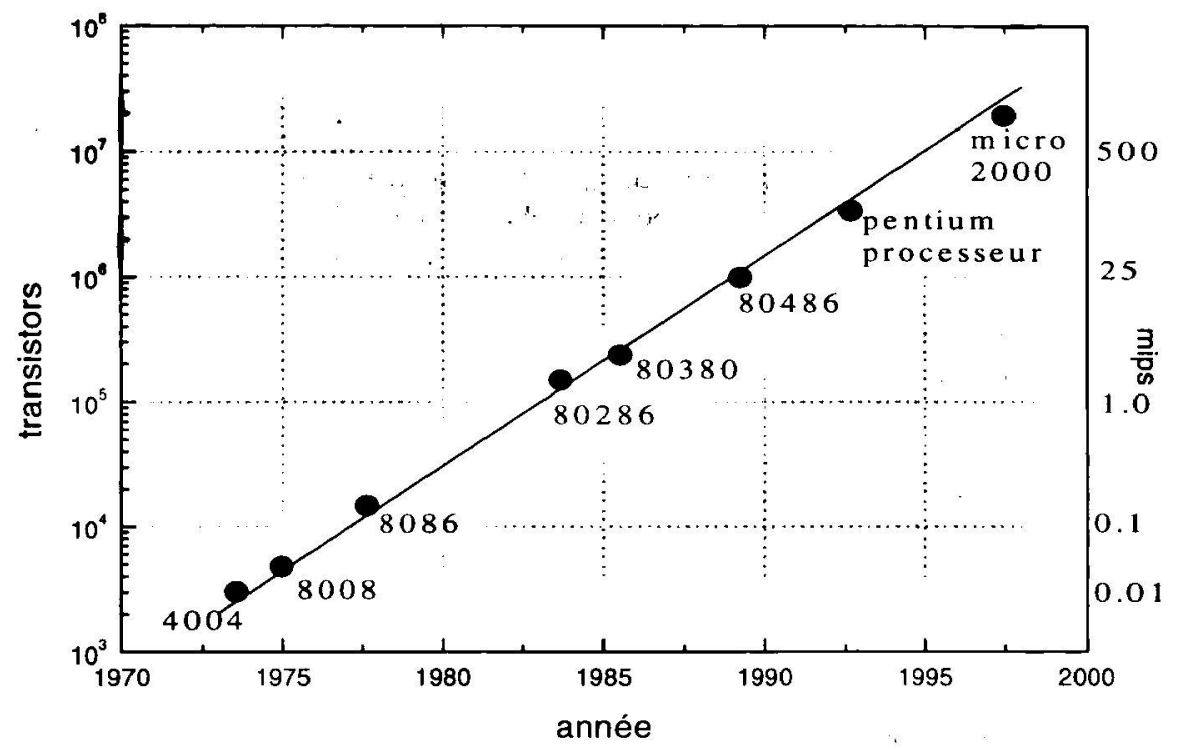

b)
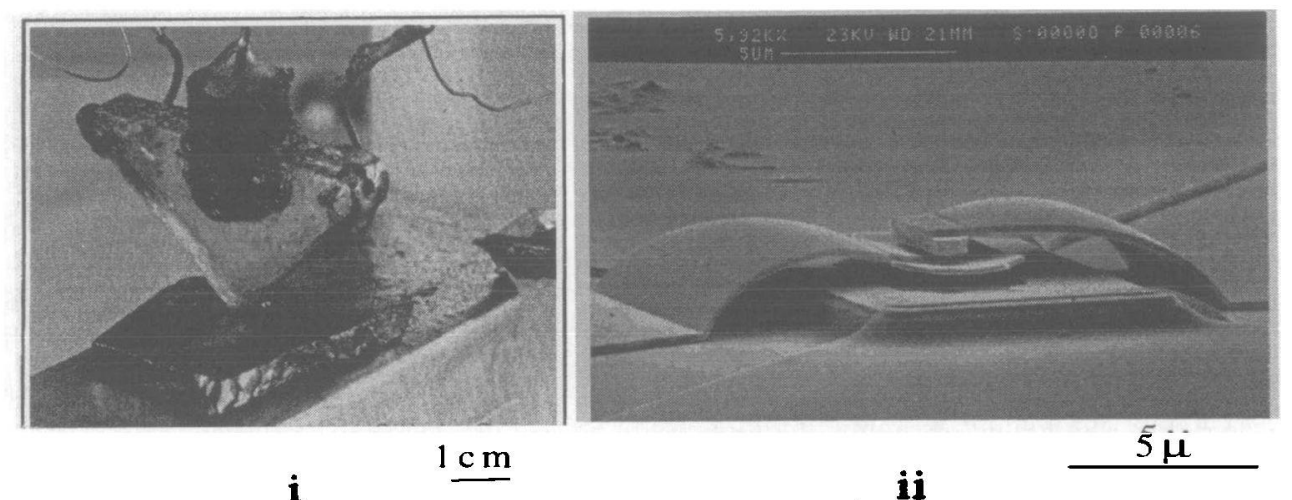

Figure 1.1 : a) Loi de Moore, donnant le nombre typique de transistors par puce au cours des années. b) Transistors : i) le premier transistor, de taille centimétrique, 1947, Bell Laboratories ii) un transistor expérimental d'aujourd'hui, le MHBT (Metal Heterojunction Bipolar Transistor), 1993, L2M-CNRS.

les semi-conducteurs. Aujourd'hui, on ne peut parler des matériaux semi-conducteurs sans y associer les microstructures qu'il est possible de réaliser avec ; ce qui est vrai, aussi, dans le domaine de l'optique. D'autre part, les applications envisagées pour les propriétés optiques des semi-conducteurs sont de plus en plus nombreuses. Enfin, la démarche du physicien s'en 
est trouvée renouvelée et les semi-conducteurs ont ouvert la voie à une généralisation de cette démarche dans les autres familles de matériaux, en particulier les métaux magnétiques.

\subsection{Propriétés électriques}

La théorie quantique des solides, qui dans un premier temps se place dans le cadre d'une approximation à un électron, établit donc que les états électroniques y sont groupés en bandes, ce qui les oppose aux atomes. Dans ces bandes, les divers états se distinguent par un vecteur d'onde $\mathbf{k}$, assimilable au moment cinétique. Dans un semi-conducteur, ou un isolant, la dernière bande peuplée (bande de valence, BV) l'est complètement, ce qui entraîne, par le principe de Pauli, qu'il n'y a pas place pour un changement d'état, donc un changement de moment tel qu'une accélération provoquée par un champ électrique. Quant à la première bande vide (bande de conduction, BC), elle l'est aussi complètement, et donc, il n'y a rien à accélérer. La température, ou l'excitation optique par des photons d'énergie supérieure au gap, activant la promotion d'électrons de la $\mathrm{BV}$ à la $\mathrm{BC}$, changent en principe cette situation de blocage électrique. Mais c'est surtout le dopage avec des impuretés électriquement actives qui est d'une immense importance pratique.

Le paradigme du donneur est un atome d'arsenic noyé dans le cristal de silicium, et occupant la place d'un atome du matériau hôte, tétravalent. Étant pentavalent, une de ses liaisons ne sera pas satisfaite. Il dispose donc d'un électron supplémentaire, qui se trouve être faiblement lié. À température finie, cet électron sera donc disponible pour peupler des états de conduction. Pour décrire la situation (Cf. fig.1.2), on représente les états électroniques supplémentaires par un niveau "donneur", situé un peu en dessous de la bande de conduction, et donc susceptible d'être vide à température ambiante. De façon symétrique, un accepteur, par exemple du bore dans $\mathrm{Si}$, liera un trou à l'état neutre, mais sera susceptible d'être ionisé à température ambiante, autorisant un courant électrique de trous. Ces états localisés ne sont évidemment pas caractérisés par un $\mathbf{k}$ unique comme les

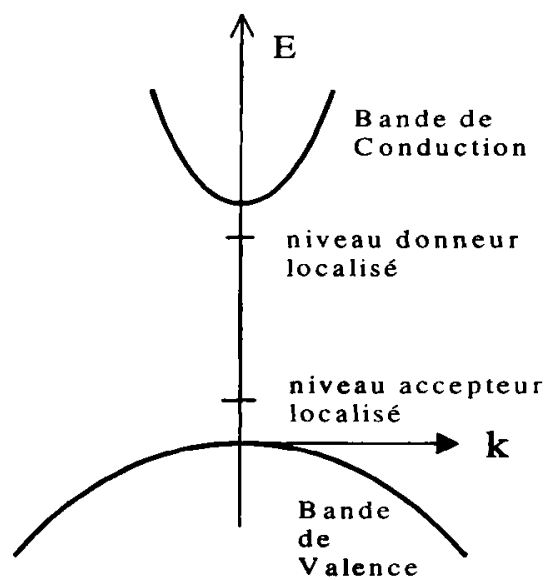

Figure 1.2 : Représentation des états électroniques du semi-conducteur dans un schéma à un électron, sur des "courbes de dispersion": énergie E en fonction du vecteur d'onde $k$. Les niveaux d'impureté localisés n'ont pas de $\mathbf{k}$ bien défini. états des bandes. Cependant, ils sont souvent d'énergie proche d'un extremum de bande, et sont donc construits par combinaison linéaire des états de bande avec une forte prédominance des états proche de l'extremum. Ils peuvent alors être décrits dans le cadre de l'Approximation de la Fonction Enveloppe (EFA) que nous utiliserons sur l'exemple des hétérostructures bidimensionnelles (2D).

Une fois connues les densités d'états de valence et de conduction d'une part, de donneurs et d'accepteurs d'autre part, la répartition des charges à l'équilibre se décrit donc à toute température par une fonction d'occupation de Fermi-Dirac. Elle se caractérise très synthétiquement par une température, bien entendu, et par un potentiel chimique, couramment 
appelé niveau de Fermi, $\mathrm{E}_{\mathrm{F}}$. Pour l'essentiel, on peut retenir que $\mathrm{E}_{\mathrm{F}}$ se situera aux environs de la BC dans un matériau dopé par des donneurs (de type $n$ ), et de la bande de valence s'il est dopé par des accepteurs (de type p). II n'est jamais inutile, même pour un opticien, de comparer le dopage de son matériau, ou ce qu'il peut en connaître, aux densités de photoporteurs créés par l'illumination.

Dans un matériau pur, intrinsèque, $\mathrm{E}_{\mathrm{F}}$ sera situé dans le gap, équilibrant les densités d'électrons et de trous, en tenant compte des densités d'états différentes. Mais cette dernière situation est moins courante que celle des matériaux "semi-isolants", où ont été introduits plus ou moins volontairement des centres profonds, qui fournissent une densité suffisante de niveaux isolés dans le gap pour interdire la conduction. En effet, mis à part le silicium, dont la technologie est très bien contrôlée, le dopage des semi-conducteurs courants comme GaAs ne se contrôle bien que dans les gammes $10^{15} \mathrm{~cm}^{-3} \mathrm{a} 10^{19} \mathrm{~cm}^{-3}$. Quant aux plus exotiques, il faut être prudent et modeste. Dans le cas de couches minces $(\sim 1 \mu \mathrm{m})$ épitaxiées sur un substrat nécessairement beaucoup plus épais $(500 \mu \mathrm{m})$, c'est dans la couche mince qu'a lieu l'effet physique intéressant pour la recherche fondamentale (effet Hall quantique) ou pour les applications (transistor). Il est donc impératif d'assurer que le courant ne passe pas dans le substrat. C'est une des servitudes de la technologie.

Une autre de ces servitudes, que les opticiens ont parfois tendance à oublier, est l'existence d'une surface assez mal contrôlée elle aussi. À la surface d'un semi-conducteur, les états électroniques sont modifiés profondément: liaisons pendantes, éventuelles reconstructions (ces liaisons pendantes se réarrangent), adsorption d'éléments étrangers (l'oxygène est un bon candidat), tout cela nous permet tout juste de soupçonner l'immense champ d'intérêt de la physique des surfaces [8]. Mais tout cela peut dépendre des conditions de préparation, de conservation de l'échantillon... en bref, c'est assez incontrôlable, et la passivation des surfaces de semi-conducteurs est un sujet de recherche en soi.

\subsection{Un exemple de dispositif électronique : le MES-FET GaAs}

Plutôt que d'explorer toutes les configurations possibles, ce qui irait bien au-delà des ambitions de cette présentation, nous pouvons nous intéresser au fonctionnement d'un dispositif électrique parmi d'autres, qui nous donnera un aperçu des possibilités offertes par la microélectronique, nous permettra de voir concrètement comment utiliser la notion de niveau de Fermi et introduira une mise en garde aux opticiens.

Il s'agit d'un transistor à effet de champ de type "électrode métallique/semiconducteur”, plus connu sous son acronyme anglais MES-FET (Cf. fig. 1.3 a). Il s'agira de GaAs, un matériau effectivement utilisé en microélectronique (beaucoup moins que le silicium), mais que nous rencontrerons souvent dans ce cours d'optique. La couche active est épaisse, par exemple de $1 \mu \mathrm{m}$, dopée $\mathrm{n}$, typiquement à $10^{17} \mathrm{~cm}^{-3}$. Pour qu'elle soit effectivement la seule couche active électriquement, nous avons déjà vu qu'il faut qu'elle soit déposée sur un substrat semi-isolant ou, dans le cas présent, de type p. Le niveau de Fermi s'égalisant entre couche active et substrat, la bande de conduction remontera vers l'intérieur de la structure, formant barrière au passage des électrons (Cf. fig. $1.3 \mathrm{~b}$ ).

Que se passe t-il maintenant à la surface? Dans GaAs, il se trouve le plus souvent que la surface comprend de très nombreux états situés à peu près au milieu du gap, disons $V_{S}$ en dessous de la bande de conduction ; par très nombreux, il faut entendre une densité surfacique suffisante pour fixer le niveau de Fermi à cette énergie, quel que soit l'apport (réaliste) d'électrons dû au dopage. Il en résulte que la couche active doit fournir des électrons pour 
maintenir l'équilibre, sur une profondeur dépendant du dopage. C'est la couche désertée, ou zone de charge d'espace.

Pour estimer sa profondeur, et le champ électrique qui y règne, plaçons nous dans l'approximation la plus simple : la densité de charge est pour l'essentiel celle des donneurs
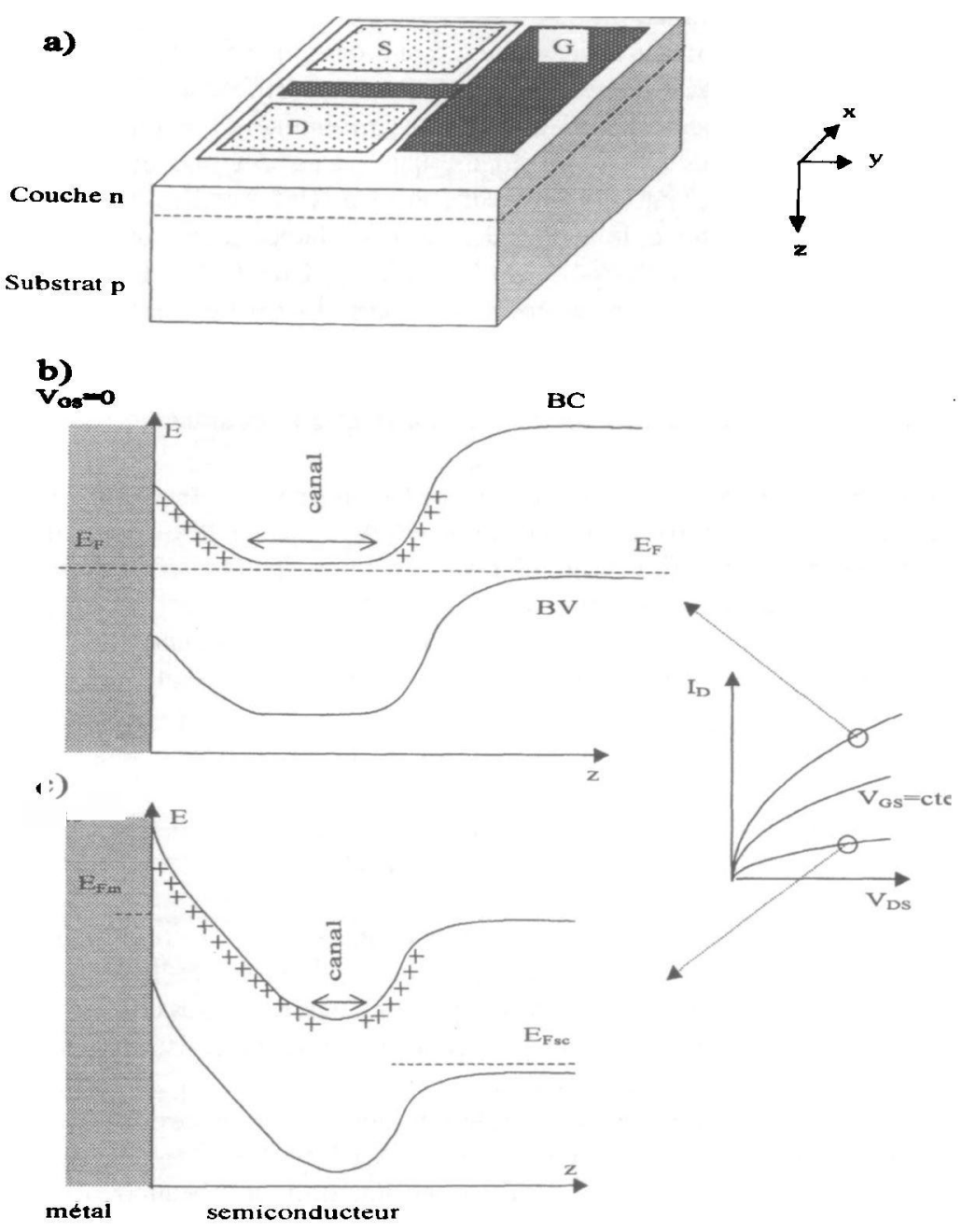

Figure 1.3 : a) Schéma d'un MES FET. On distingue la source $S$ et le drain $D$ reliés par le canal C, qui passe sous la grille $G$. b) Profil de bande en fonction de la profondeur $z$ à $V_{G}=0$ : la région $n$ conduit. c) Profil de bande a $\mathrm{V}_{\mathrm{G}}<0:$ la région $\mathrm{n}$ est désertée.

ionisés : $\rho=+\mathrm{eN}_{\mathrm{d}}^{+}$. En intégrant deux fois l'équation de Poisson, la chute de potentiel dans la zone désertée est donc une fonction quadratique de la profondeur $\mathrm{z}$ :

$\mathrm{V}=\left(+\mathrm{eN}+/ 2 \varepsilon \varepsilon_{0}\right)\left(\mathrm{z}-\mathrm{z}_{0}\right)^{2}$ où la profondeur de la couche désertée est $\mathrm{z}_{0}$. On en déduit :

$$
\mathrm{z}_{0}=\left(2 \mathrm{~V}_{\mathrm{S}} \varepsilon \varepsilon_{0} / \mathrm{eN}+{ }_{\mathrm{d}}^{+}\right)^{1 / 2}
$$


Pour fixer les idées, on peut noter que, dans $\mathrm{GaAs}, \mathrm{V}_{\mathrm{s}}$ est de l'ordre de $0.7 \mathrm{eV}$, et $\mathrm{z}_{0}$ vaut environ $0.1 \mu \mathrm{m}$ pour un dopage $\mathrm{N}_{\mathrm{d}} \sim 10^{17} \mathrm{~cm}^{-3}$.

Le transistor est fabriqué à partir d'une telle couche (Cf. fig. $1.3 \mathrm{a}$ ). On la contacte (source et drain) avec des électrodes métalliques déposées sur des parties surdopées localement à quelques $10^{18} \mathrm{~cm}^{-3}$, et généralement recuites pour fournir un contact ohmique. La physique des contacts est complexe, mais on peut se contenter de vérifier que le courant passe... Par contre, l'électrode de commande, la grille, consiste en un dépôt métallique plus délicat, qui va respecter l'intégrité du semi-conducteur. À l'équilibre, c'est-à-dire sans tension de grille appliquée, les deux niveaux de Fermi du métal et du semi-conducteur vont s'aligner. En l'occurrence, sur GaAs, il reste fixé sur les états de surface du milieu du gap. Chaque matériau fournira donc les charges nécessaires à cet équilibre. Du côté semi-conducteur, cela amène la création de la zone de charge d'espace, comme on vient de le voir. Du côté du métal, la densité électronique est de l'ordre de $10^{22} \mathrm{~cm}^{-3}$; donc les perturbations de la structure de bande y sont imperceptibles. Il résulte de cet équilibre la formation d'un profil de potentiel pour les électrons très asymétrique : il s'agit d'une diode Schottky, redresseuse car le courant reste faible sous tension de grille négative, et croît rapidement sous tension positive. Le fonctionnement du dispositif est simple à comprendre dans son principe. Une tension négative appliquée sur la grille n'injectera que peu de porteurs, mais déséquilibrera les deux niveaux de Fermi, qui restent définis dans le métal d'une part, et dans la profondeur du semi-conducteur d'autre part, car tant qu'il reste des charges mobiles, le champ est négligeable. Ce n'est pas le cas dans la zone désertée, hors d'équilibre. Celle-ci s'élargira, diminuant la conductivité (Cf. fig. $1.3 \mathrm{c}$ ). On peut donc contrôler, pour l'essentiel, un courant avec une tension sous haute impédance.

Cette discussion nous permet d'introduire une mise en garde. En se référant à l'expression (1.1), on voit que la zone de charge d'espace d'une structure GaAs varie de, typiquement, $1 \mu \mathrm{m}$ pour un dopage de $10^{15} \mathrm{~cm}^{-3}$, à $30 \mathrm{~nm}$ pour $10^{18} \mathrm{~cm}^{-3}$. Et le champ électrique au milieu de cette zone, variera corrélativement de $10 \mathrm{kV} / \mathrm{cm}$ à $300 \mathrm{kV} / \mathrm{cm}$. Ce champ peut être non négligeable dans la discussion de nombreux effets électro-optiques et non linéaires. Surtout, il est susceptible d'être écranté par la photo-injection de porteurs ; il suffit pour cela que la densité injectée soit comparable à la densité d'impuretés. Ce sera encore une source de non-linéarité ; encore faut-il en être conscient. L'exemple que nous venons de donner concerne un matériau bien contrôlé, bien connu, probablement le meilleur de ce point de vue hormis le silicium. Dans la plupart des autres cas, on ne pourra pas en savoir tant. Mais un problème mal contrôlé ne peut pour autant être ignoré.

\subsection{Propriétés optiques des semi-conducteurs}

Chacun sait que les semi-conducteurs sont transparents pour les photons d'énergie inférieure au gap. Les semi-conducteurs sont donc d'abord des isolants, avec un gap entre les derniers états peuplés et les premiers états vides (si l'on néglige les états d'impuretés). Mais, de même que pour leurs propriétés électriques, les propriétés optiques des semi-conducteurs usuels, reposent sur l'existence d'états électroniques bien délocalisés. C'est ce qui fait cet ensemble de matériaux si riche et qui a donné lieu à tant d'études originales. Ainsi, les excitations élémentaires du cristal seront au premier ordre, des paires électron-trou. De même, un état lié de donneur ou d'accepteur, relativement peu profond, sera assez semblable aux états 
délocalisés (au sens où l'EFA y est appliquable), et relèvera de l'approche de type "semiconducteurs", à laquelle est consacré ce cours.

Par contre, les états d'impuretés "profondes" dans les solides, ce qui veut dire d'énergie très différentes des bandes, ne gardent plus guère de souvenir des états délocalisés des bandes. II faut, pour rester pertinent, les considérer plutôt comme des atomes, isolés, mais placés dans un environnement de symétrie réduite. Dans la tradition académique, ils relèvent plutôt de "l'optique des solides".

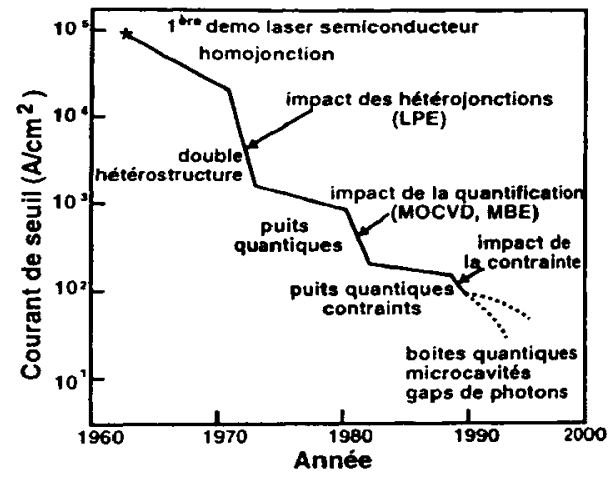

Figure 1.4 : Évolution de la densité du courant de seuil des lasers à semi-conducteurs au cours des années. (D'après C.Weisbuch, [10])

Au plan des applications, il faut d'abord citer le laser à semi-conducteurs, lui aussi très différent du laser solide de type YAG, par, justement, l'organisation des états en bandes qui permet le pompage électrique direct. Les mérites du laser à semi-conducteurs sont la miniaturisation et la rapidité de modulation, qui ouvrent à l'optique tout un champ d'applications pour la transmission de l'information. Ces qualités essentielles sont liées à la densité de courant au seuil, dont on peut mesurer les progrès sur la figure 1.4, progrès essentiellement dus à l'utilisation d'hétérostructures. Il a été décidé, dans cette École d'Été de ne pas aborder le sujet des lasers à semi-conducteurs, déjà bien traité dans le même cadre [9]. Mais du traitement optique du signal, il sera par contre beaucoup question dans cette École, puisque c'est un domaine d'applications important pour l'optique non linéaire, avec ou sans fibres optiques. Enfin, il ne faut pas négliger les applications des semi-conducteurs dans le domaine de l'affichage, qui pourrait représenter des marchés considérables et pourtant mettre en jeu les derniers développements de la physique et de la technologie.

Il est courant, en matière d'optique, de comparer les semi-conducteurs aux atomes. Évidemment, on gagne en compacité pour l'interaction entre matière et rayonnement. De plus, on le sait, la propagation du champ dans la zone de transparence est aussi considérablement affectée par la densité de matière, et on en rend compte par un indice de réfraction. Nous verrons aussi qu'on trouve, dans les semi-conducteurs, une situation de couplage fort avec la lumière sans équivalent dans les milieux plus dilués ( $\mathrm{Cf}$. la soussection 4.7 sur les polaritons excitoniques). Dans la zone de transparence, lorsqu'on reste suffisamment éloigné de la résonance avec le gap, beaucoup de propriétés optiques sont assez bien décrites par un modèle d'oscillateur de Lorentz, auquel sont éventuellement associées des non-linéarités. On peut à ce propos se demander ce que devient la notion de force d'oscillateur dans un solide.

Dans un atome, la force d'oscillateur $f_{i f}$ associée à une transition d'énergie $E_{i f}$, notion éminemment quantique, représente, en quelque sorte, la "fraction d'électron" de l'oscillateur de Lorentz classique affectée à cette transition particulière. La susceptibilité optique est

$$
\chi(\omega)=-\frac{n e^{2}}{m \varepsilon_{0}} \sum_{i, f} \frac{f_{i f}}{\omega^{2}-\omega_{i f}^{2}+2 i \gamma_{i f} \omega_{i f}} \text { avec la règle de somme }: \sum_{f} f_{i f}=1 \text {, dont la }
$$

signification est claire : la force d'oscillateur totale, associée à l'ensemble des transitions du 
système quantique, rejoint celle de l'oscillateur classique. Dans un atome, une transition particulière possède donc une force d'oscillateur (quantité sans dimension) $f \sim 0,1$.

Un semi-conducteur est constitué d'environ $\mathrm{N}_{\text {at }}=10^{23}$ atomes $/ \mathrm{cm}^{3}$. Les transitions optiques entre bande de valence et bande de conduction peuvent, dans une certaine mesure, être vues comme dérivées des transitions atomiques. Donc, elles sont affectées d'une force d'oscillateur globale, par unité de volume, de l'ordre de $\mathrm{f} . \mathrm{N}_{\mathrm{at}} \sim 10^{22} \mathrm{~cm}^{-3}$. Mais ces transitions, parce que nous sommes dans un semi-conducteur, sont élargies comme les bandes, de l'ordre de $\Delta B \approx 10 \mathrm{eV}$. En d'autres termes, la force d'oscillateur $f$ "par atome" se trouve diluée sur chaque site en une force d'oscillateur par paire électron-trou (accessible optiquement). In est donc souvent plus utile d'introduire la notion de force d'oscillateur par unité de volume et d'énergie. En moyenne, elle est de l'ordre de $10^{21} \mathrm{~cm}^{-3} \mathrm{eV}^{-1}$. Mais, la densité d'état de paires électron-trou n'est pas constante. On le verra, elle varie en bas de bande avec l'énergie comme $E^{1 / 2}$ dans les matériaux massifs (3D). En supposant cette variation sur toute la largeur de bande (ce qui est osé), on montre facilement que, dans un intervalle d'énergie $\Delta \mathrm{E}$ au dessus du gap, se trouve répartie une force d'oscillateur $F_{\Delta E}=f . N_{a t}(\Delta E / \Delta B) . F$ sera donc $3.10^{18} \mathrm{~cm}^{-3}$ pour les premiers $10 \mathrm{meV}, 10^{19} \mathrm{~cm}^{-3}$ pour les premiers $100 \mathrm{meV}$.

Nous le verrons, cette force d'oscillateur de bas de bande est en fait regroupée sur une transition excitonique discrète, située à la distance $\mathrm{R}$ ( le Rydberg ) au dessous du gap. Lui est associée une force d'oscillateur suivant la loi ci dessus, soit : $F_{\text {exciton }}=f \cdot N_{a t}(R / \Delta B)^{3 / 2}$. Au passage de cette transition, on trouve donc un pic d'absorption et l'effet sur l'indice de réfraction est notable. Il s'agit là d'un effet fondamental pour les propriétés optiques des semiconducteurs du moins à basse température. Ces dernières années, la réalisation d'hétérostructures a rendu possible l'observation de cet effet à température ambiante dans beaucoup de matériaux où ce n'était pas le cas, en particulier les semi-conducteurs III-V. L'effet a donc été a popularisé bien au-delà du cercle étroit des spécialistes fondamentalistes, jusque dans le domaine de la physique du dispositif.

Quel est le domaine de validité du modèle d'oscillateur de Lorentz pour un semiconducteur? Autrement posée, la question devient : quand peut-on moyenner les subtilités de la structure de bande? Ce sera le cas loin de la résonance avec le gap, ou bien aux échelles de temps très courtes, lorsque l'incertitude en énergie rend floues les dites subtilités. Le même type de raisonnement pourra s'appliquer pour savoir jusqu'à quel point il convient de connaître le détail des niveaux des hétérostructures. On en déduit a contrario que toute la richesse des matériaux semi-conducteurs et de leurs hétérostructures s'exprimera en optique non linéaire au voisinage des résonances et aux échelles de temps relativement longues.

\section{STRUCTURE DE BANDE ET PROPRIÉTÉS GÉNÉRALES DES SEMI- CONDUCTEURS MASSIFS}

Après les considérations de symétrie qui vont suivre, nous pourrions dans le cadre restreint de ce cours considérer la structure de bande des semi-conducteurs comme donnée par des calculs et des expériences qui ne nous intéressent que secondairement. Il est cependant utile pour l'optique d'avoir une vision qualitative de l'origine des bandes. C'est pourquoi je présenterai d'abord la méthode des "liaisons fortes", avant de donner une classification grossière des divers matériaux semi-conducteurs. 
Plus tard, nous verrons que la démarche de "lingénieur en structure de bande" est au coeur de la physique des hétérostructures. Pour cet ingénieur, il faut des outils quantitatifs, même s'ils sont de validité plus limitée. Ce sont la méthode de perturbation k.p de Kane et le hamiltonien de Luttinger, qui seront présentés au paragraphe 3.3.

a)

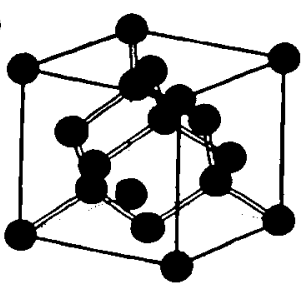

- C

b)

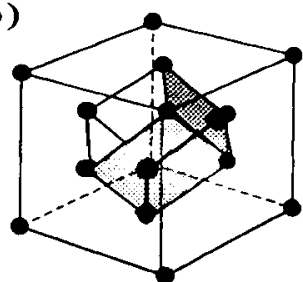

c)

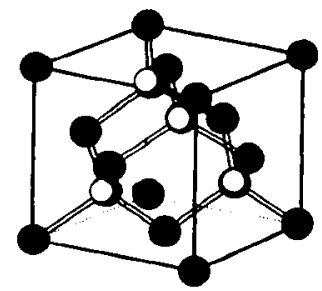

- Zn

O S

d)

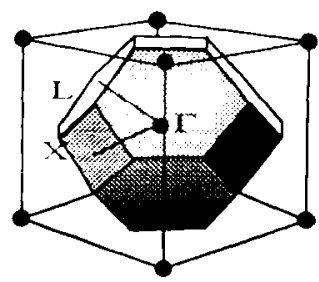

Figure 2.1 : a) La structure cristalline du diamant (mais aussi de Si et Ge) est formée de deux réseaux cubiques à faces centrées (cfc) décalés. b) Un réseau cfe avec sa cellule unité, contient donc deux atomes dans le diamant.

c) Ces deux sous-réseaux portent des atomes différents dans le cas de la blende de zinc (mais aussi de GaAs, $\mathrm{CdTe}, \ldots$ ). d) Le réseau réciproque est cubique centré. Notez les points de haute symétrie $\Gamma, X, L$, au centre ou au bord de la $1^{\text {ere }}$ zone de Brillouin (Emprunté à Yu et Cardona[6]).

\subsection{Considérations de symétrie}

Pour aborder le problème de la structure électronique des semi-conducteurs, il est d'usage, comme en physique atomique, de se placer d'abord dans le cadre de l'approximation à un électron. Cette approximation, où l'action des noyaux et des autres électrons est représentée par un champ moyen est étonnamment efficace. On met ensuite en avant la périodicité du réseau pour expliquer le regroupement des états électroniques en bandes. Ce n'est peut-être pas vraiment indispensable, car les alliages désordonnés et même les matériaux amorphes ont des structures de bandes finalement assez proches des solides parfaits; mais c'est bien commode pour préciser la forme générale des fonctions d'onde, grâce au théorème de Bloch.

Dans un cristal, par définition, le potentiel effectif $V$ auquel est soumis l'électron est périodique, ce qui s'exprime par $\mathrm{V}\left(\mathbf{r}+\mathbf{R}_{\mathbf{n}}\right)=\mathrm{V}(\mathbf{r})$ où $\mathbf{r}$ est le vecteur position de l'électron, et $\mathbf{R}_{\mathbf{n}}$ un vecteur quelconque de translation qui laisse le réseau invariant. On sait que cette 
proposition est équivalente à $\mathbf{R}_{\mathbf{n}}=\sum_{\mathbf{i}} \mathrm{n}_{\mathbf{i}} \mathbf{a}_{\mathbf{i}}$ où les $\mathbf{a}_{\mathbf{i}}$ sont les vecteurs de base d'une cellule unité (ou "maille") et les $n_{\mathbf{i}}$ sont des entiers (Cf. fig. 2.1 a et b. Notez que la maille n'est pas le cube).

Le hamiltonien total, $\mathbf{H}=\mathbf{p}^{2} / 2 \mathrm{~m}_{0}+\mathrm{V}(\mathbf{r})$ ayant la même propriété d'invariance par translation que $\mathrm{V}(\mathbf{r})$, le théorème de Bloch n'exprime rien d'autre que la proposition suivante : il existe un ensemble de fonctions propres de l'énergie et des translations du réseau. Des propriétés du groupe des translations du réseau, on déduit aisément [YC p.16] que les valeurs propres des translations $T\left(\mathbf{R}_{\mathbf{n}}\right)$ affectent la forme $\exp \left(\mathbf{i k .} \mathbf{R}_{\mathbf{n}}\right.$ ), où $\mathbf{k}$, vecteur d'onde, est défini à un vecteur du réseau réciproque près. La notion de réseau réciproque s'impose facilement dans le problème. C'est le réseau des vecteurs d'onde $\mathbf{g}$ tels que $\mathbf{g} \cdot \mathbf{R}_{\mathbf{n}}=2 \pi \mathrm{N}$ ( $\mathrm{N}$ entier). L'espace des $k$ est donc lui aussi périodique, et l'on connaitra toute la physique en n'explorant qu'une seule cellule unité du réseau réciproque, la zone de Brillouin (ZB). On trouvera sur la figure $2.1 \mathrm{~d}$ une esquisse de la $1^{\text {ere }}$ zone de Brillouin d'une structure blende de zinc faisant apparaître quelques points remarquables qu'il est utile de savoir repérer.

Si l'on choisit donc comme base l'ensemble commode formé par les fonctions propres des translations, chaque élément $\psi(r)$ en sera caractérisé :

- par les valeurs propres des opérateurs translations $\exp \left(\mathbf{i k .} \mathbf{R}_{\mathbf{n}}\right)$.

- par d'autres nombres quantiques, symbolisés par l'indice $\lambda$.

Alors, $\psi_{\lambda, \mathbf{k}}\left(\mathbf{r}+\mathbf{R}_{\mathbf{n}}\right)=$ eik.r $^{i k, \mathbf{k}}(\mathbf{r})$, qui ne diffère de $\psi_{\lambda, \mathbf{k}}(\mathbf{r})$ que par un facteur de phase, et qui est aussi fonction propre du hamiltonien avec la même valeur propre.

Satisfont à cette relation plusieurs formes de fonctions d'onde commodes, et d'abord la fonction "de Bloch" :

$$
\psi_{\lambda, \mathbf{k}}(\mathbf{r})=V^{-1 / 2} \mathrm{e}^{\mathrm{ik} \cdot \mathbf{r}} \mathrm{u}_{\lambda, \mathbf{k}}(\mathbf{r})
$$

où $u_{\lambda, \mathbf{k}}(\mathbf{r})$ est invariante par les translations $T\left(\mathbf{R}_{\mathbf{n}}\right)$, donc a la même périodicité que le cristal. De plus, $\mathrm{V}$ est le volume du cristal, et la fonction d'onde est normalisée à 1 sur ce volume, ce qui impose la normalisation des $\mathbf{u}_{\lambda, \mathbf{k}}(\mathbf{r})$ à 1 sur la maille élémentaire.

Pour prendre en compte le caractère fini du cristal, il est traditionnel d'imposer des conditions aux limites périodiques, du type $\psi \lambda, \mathbf{k}\left(\mathbf{r}+\mathbf{L}_{\mathbf{i}}\right)=\psi \lambda, \mathbf{k}(\mathbf{r})$, où $\mathbf{L}_{\mathbf{i}}=\mathrm{N}_{\mathrm{i}} \mathbf{a}_{\mathbf{i}}$ est la longueur totale du cristal dans la direction i. Cette démarche, dite de Born-von Karman (BVK), est la plus élégante au plan mathématique pour rendre compte des situations physiques où la taille (macroscopique) du cristal ne peut influer sur les propriétés microscopiques. Comme souvent, il faudra faire preuve d'un peu de sens physique pour trouver les critères du «macroscopique ». Ce sera le cas lorsque la cohérence quantique des fonctions d'onde ne peut être maintenue en pratique sur aucune des dimensions du cristal. Dans le cas contraire, où la taille du cristal est plus petite, au moins dans une direction, que cette longueur de cohérence, tout en restant assez grande par rapport à la maille élémentaire pour justifier l'approche de Bloch, on parle de méso-, micro- ou nano-structures. Alors, il faut bien sûr imposer aux fonctions d'onde des conditions aux limites adéquates : pour un nanocristal isolé, ce sera l'annulation aux bords ; dans les hétérostructures, comme nous le verrons, ce seront d'autres plus subtiles mais qualitativement équivalentes.

En tout cas, l'effet de ces conditions aux limites est de limiter la densité des k possibles et donc, à l'intérieur d'une zone de Brillouin, de limiter leur nombre. On montre aisément que le nombre total de $\mathrm{k}$ possibles et physiquement différents est égal au nombre de cellules unités. 
La première $\mathrm{ZB}$ est de dimension typique $2 \pi / \mathrm{a}$, où a est la dimension de la maille élémentaire. Ces remarques permettent de connaître la densité d'états $\rho$ par unité de volume de l'espace réciproque. On se restreindra pour l'instant à un matériau massif, $3 D$. Alors : $\rho(k)=(L / 2 \pi)^{3}$, de sorte qu'on a bien : $\int_{1 \text { ère } Z B} \rho(k) d^{3} \mathbf{k}=(L / a)^{3}$.

La densité d'états par unité d'énergie est une autre quantité importante. En supposant par anticipation une loi de dispersion parabolique $E=\hbar^{2} \mathbf{k}^{2} / 2 \mathrm{~m}$, il vient :

$$
\rho(E) d E=(L / 2 \pi)^{3} 2 \pi\left(2 \mathrm{~m} / \hbar^{2}\right)^{3 / 2} \mathrm{E}^{1 / 2} \mathrm{dE}
$$

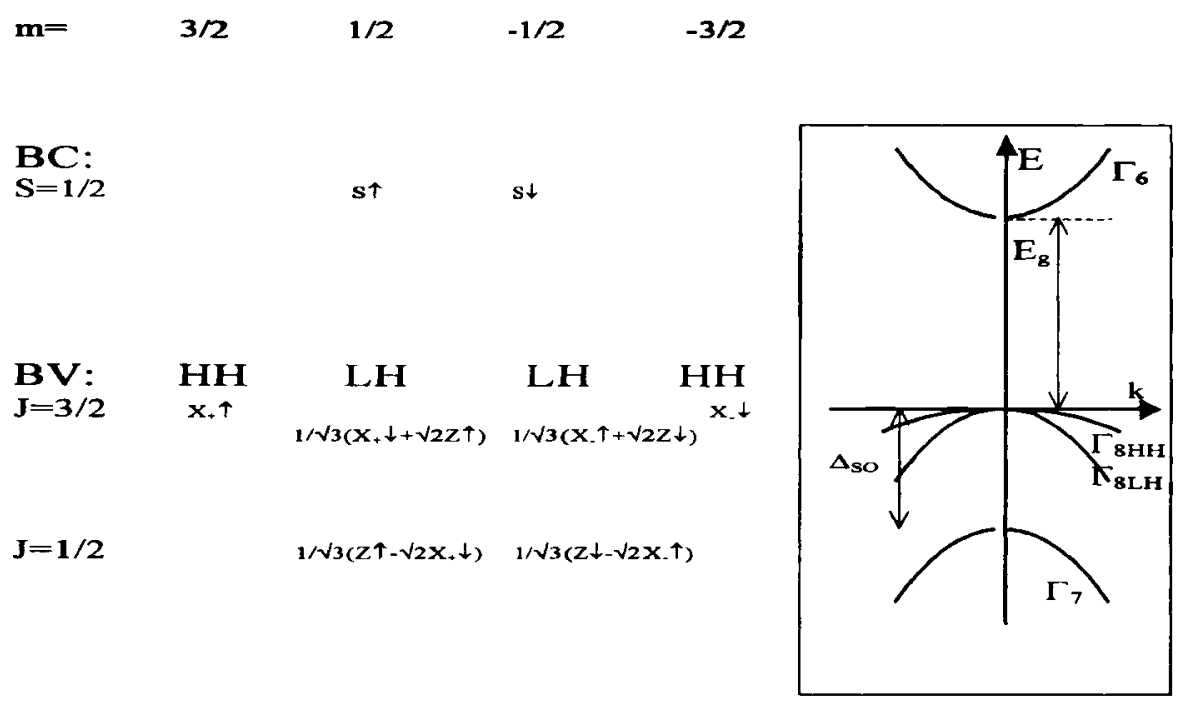

Figure 2.2 : Fonctions propres du moment angulaire total, formant la base des états électroniques de centre de zone dans les semi-conducteurs cubiques usuels de type $\mathrm{ZnS}, \mathrm{GaAs}, \ldots$ On a posé $\mathrm{X} \pm=(\mathrm{X} \pm \mathrm{iY}) / \mp \sqrt{2}$. La $\mathrm{l}^{\text {tro }}$ bande de valence $(\mathrm{J}=3 / 2)$ est dégénérée en $k=0$, mais se sépare en trous lourds $(\mathrm{HH})$ et légers $(\mathrm{LH})$ en dehors.

\subsection{Aperçu sur la méthode des liaisons fortes}

Les calculs de bandes par la méthode des liaisons fortes sont fondés sur les mêmes idées que la méthode des combinaisons linéaires d'orbitales atomiques en chimie. Nous allons l'appliquer de façon irréaliste (Cf. la mise en garde 2.2.4.1), mais pédagogique, au cas d'un matériau extrêmement ionique, et avec toutes les approximations confortables, pour comprendre qualitativement la construction de la structure de bande avec le minimum d'effort.

\subsubsection{Orbitales atomiques}

Considérons donc un matériau très ionique, du type de la blende de zinc ZnS, dont la structure cristalline sert de paradigme à celle de la plupart des semi-conducteurs composés usuels. La liaison chimique peut se comprendre au premier abord en utilisant la vieille "règle de l'octet" : les deux électrons $4 s^{2}$ du zinc viennent compléter la couche $3 s^{2}, 3 p^{4}$ du soufre. On a donc une 
dernière couche pleine $\mathrm{p}^{6}$ qui va donner naissance aux bandes de valence, puis, à plus haute énergie, une première couche vide $s^{0}$ qui donnera la bande de conduction. En ce qui concerne cette dernière, les deux états par cellule unité correspondent évidemment à la dégénérescence de spin. Pour les premières, trois orbitales $p$ pointent selon les trois directions $x, y, z$, non arbitraires dans un cristal cubique. Elles sont douées d'un moment orbital et, tenant compte du spin, nous savons que les bons états sont ceux du moment angulaire total, $J=L+S$.

Les états "atomiques" de départ seront donc convenablement caractérisés par leurs deux nombres quantiques du moment angulaire total, quantifié le long de la direction $\mathrm{z}$. On les écrit comme sur la figure 2.2. Les notations qui y sont employées sont particulièrement adaptées à l'étude des transitions optiques entre bandes de valence et de conduction, avec leurs règles de sélection de polarisation. Selon les auteurs, on trouvera $s, p_{x}, p_{y}, p_{z}, p_{+}, p_{-}$, ou bien, respectivement, $\mathrm{S}, \mathrm{X}, \mathrm{Y}, \mathrm{Z}, \mathrm{X}^{+}, \mathrm{X}^{-}$, ces notations de la partie orbitale de la fonction d'onde étant éventuellement complétées par l'état de spin $\uparrow, \downarrow,(\uparrow+\downarrow) / \sqrt{2}$, etc...

Dans les atomes, déjà, la dégénérescence est levée par l'interaction spin-orbite entre les quatre états $J=3 / 2$ et les deux états $J=1 / 2$. Les premiers sont repoussés à haute énergie, et les seconds à basse énergie (on parle parfois de bande split-off). La force de l'interaction spinorbite est liée à la charge du noyau. Elle varie donc beaucoup avec le numéro atomique de l'anion et, de fait, explique en grande partie les variations de gap entre semi-conducteurs d'une même famille à cation commun.

\subsubsection{Formation des bandes}

Revenons maintenant à la formation des bandes, dans une optique de liaison forte. Toute la physique est dans le recouvrement des orbitales atomiques $\varphi_{\lambda}(\mathbf{r})$ de sites voisins, qui donne une pertinence à des combinaisons linéaires délocalisées, ayant les propriétés de symétrie appropriées à des fonctions d'onde du cristal. Compte tenu donc du théorème de Bloch, la proposition la plus simple est :

$$
\Psi_{\lambda, \mathbf{k}}(\mathbf{r})=\mathrm{L}^{-3 / 2} \sum_{\mathbf{n}} \mathrm{e}^{\mathrm{ik} \cdot \mathbf{R}_{\mathbf{n}}} \varphi_{\lambda}\left(\mathbf{r}-\mathbf{R}_{\mathbf{n}}\right)
$$

qui devrait être fonction propre du hamiltonien du cristal :

$$
\mathbf{H}(\mathbf{r})=-\hbar^{2} \nabla^{2} / 2 \mathrm{~m}_{0}+\sum_{\mathrm{i}} \mathrm{V}_{0}\left(\mathbf{r}-\mathbf{R}_{\mathbf{i}}\right)
$$

où $\mathrm{m}_{0}$ est bien la masse de l'électron libre, $\mathrm{V}_{0}$ est le potentiel "atomique", localisé, et où la somme court sur toutes les cellules du cristal. Pour simplifier à l'extrême, nous négligeons tout couplage entre différents niveaux "atomiques". C'est la raison pour laquelle nous n'avons pas sommé sur les indices de niveau $\lambda$. Nous négligerons aussi tous les termes de couplage autres qu'entre premiers voisins, et nous supposerons les fonctions atomiques orthogonales entre elles, ce qui assure que les fonctions (2.3) sont déjà correctement normées. Alors, l'énergie associée à chaque fonction propre s'écrit :

$$
E_{\lambda, k}=V^{-1} \sum_{n, m} e^{i k .}\left(R_{n}-R_{m}\right) \int \varphi_{\lambda}\left(\mathbf{r}-\mathbf{R}_{m}\right) H(r) \varphi_{\lambda}\left(\mathbf{r}-\mathbf{R}_{\mathbf{n}}\right) \mathrm{d}^{3} \mathbf{r}
$$

qui se décompose en : 


$$
\begin{aligned}
& E_{\lambda, k}=V-1 \sum_{n, m} \text { eik. }\left(R_{n}-R_{m}\right)\left[\delta _ { n , m } \left(E_{\lambda}+\right.\right. \\
& \left.\sum_{ \pm 1} \int \varphi_{\lambda}\left(r-R_{n \pm 1}\right) V_{0}\left(r-R_{n}\right) \varphi_{\lambda}\left(r-R_{n \pm 1}\right) d^{3} r\right) \\
& \left.\delta_{n \pm 1, m} \int \varphi_{\lambda}\left(\mathbf{r}-\mathbf{R}_{m}\right) V_{0}\left(r-R_{n}\right) \varphi_{\lambda}\left(\mathbf{r}-\mathbf{R}_{\mathbf{n}}\right) d^{3} \mathbf{r}\right] \\
& + \text { termes d'ordre supérieurs... }
\end{aligned}
$$

\section{Fonctions S $\mathbf{k} / / \mathbf{z}$}
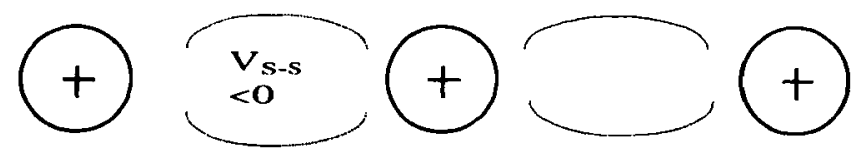

Fonctions $p_{z}$
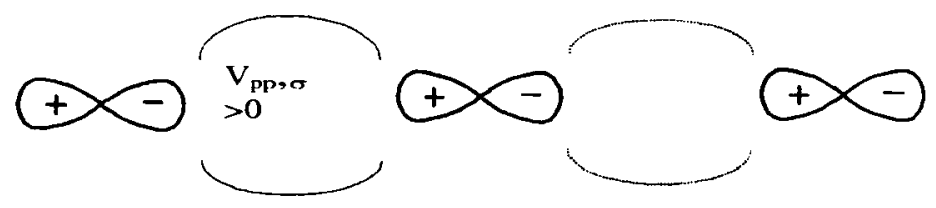

Fonctions $p_{x, y}$
$k / / z$
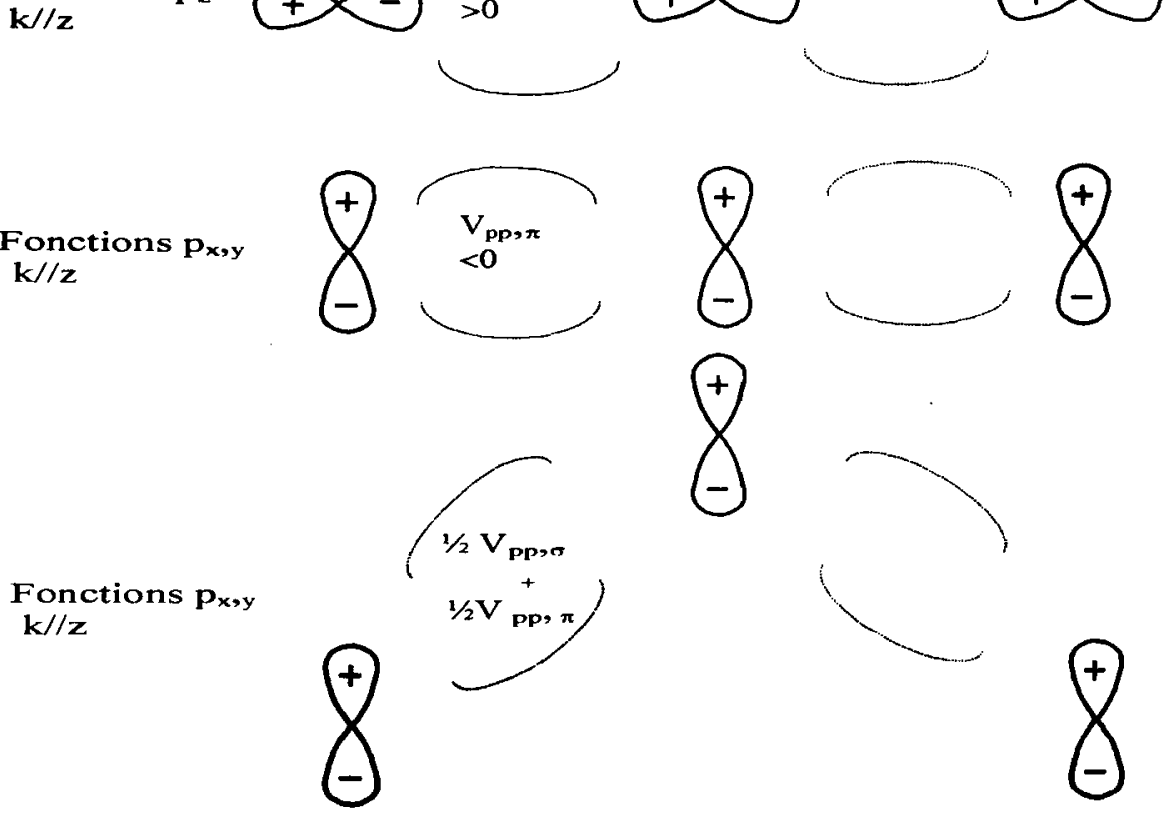

$\underset{k / / z}{\text { Fonctions }} p_{x, y}$
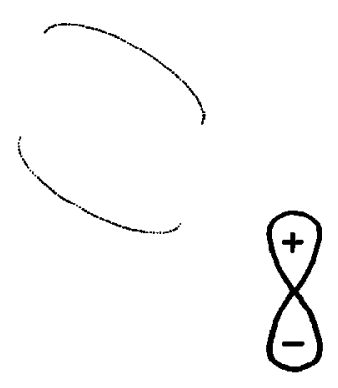

Figure 2.3 : Schéma de quelques configurations de recouvrement entre orbitales $S$ et $P$, et conséquences qualitatives sur le terme de couplage $V$.

Les deux premiers termes, diagonaux, représentent l'énergie $E_{\lambda}^{\prime}$ des états "atomiques" abaissée par le couplage. Ils sont indépendants de $\mathbf{k}$ de par la condition $\delta_{n, m}=0$ sauf $\sin =n$.

Le troisième (et on s'en tiendra là en négligeant les suivants) est non diagonal et représente le couplage $V_{\perp}$ entre premiers voisins (intégrale de recouvrement). Il dépend bien sûr du détail du potentiel, des fonctions "atomiques" de base, de leur direction (je pense aux fonctions p), et de la symétrie du réseau cristallin. Par exemple, dans le cas d'un réseau 
cubique simple et pour une direction de $\mathbf{k}$ parallèle à un des côtés de la maille, il vient facilement :

$$
E_{\lambda, k}=E_{\lambda}^{\prime}+V_{1} \sum_{n, m} \delta_{m, n \pm 1} e^{i k .\left(R_{n}-R m\right)}=E_{\lambda}^{\prime}+V_{\lambda, \mu} \cos k a
$$

où l'indice $\mu$ sera différent pour une liaison s-s, p-p, $\sigma$ ou p-p, $\pi$ (Cf. fig. 2.3).

\subsubsection{Quelques enseignements de portée générale}

On peut maintenant voir quelles leçons tirer de cette théorie très simplifiée . Pour la discussion, il est utile de visualiser la situation, schématisée sur la figure 2.3.

2.2.3.1 Pour chaque niveau atomique, ou moléculaire, discret, les $\mathrm{N}$ états localisés dégénérés des atomes découplés se sont répartis, avec l'introduction du couplage, en $\mathbf{N}$ états délocalisés, repérés par un indice $\mathrm{k}$ qui a la signification d'un vecteur d'onde. Pour s'en convaincre, observons la fonction d'onde de type liaison forte (éq.2.3), elle-même très proche d'une forme "de Bloch" eik.r $\sum_{\mathbf{n}} \varphi_{\lambda}\left(\mathbf{r}-\mathbf{R}_{\mathbf{n}}\right)$. Seule la partie exponentielle dépend explicitement de k. Aux

faibles valeurs de $\mathrm{k}$, elle est lentement variable à l'échelle de la maille, au contraire de la partie périodique. Tout se passe donc comme si l'électron sautait d'un site à l'autre. Cependant, la relation $\mathrm{E}(\mathbf{k})$, parabolique aux petits $k$, s'exprime par une masse effective, qui n'a rien à voir avec celle de l'électron libre, mais dépend du recouvrement $\mathrm{V}$ :

$$
\mathrm{m}^{*}=\hbar^{2} / 2 \mathrm{~V}_{\lambda, \mu} \mathrm{a}^{2}
$$

On le voit, un fort couplage entre atomes adjacents induit une grande largeur de bande, une faible masse effective, et donne plus de "corps physique" à cette réalité abstraite des états délocalisés. J'y reviens, c'est sans doute là que réside le bon critère de distinction entre semiconducteur et isolant, plus que dans la largeur du gap.

2.2.3.2 De même que l'hybridation des orbitales dans une molécule diatomique donne deux états liant et antiliant d'énergies différentes, de même les $\mathrm{N}$ états de Bloch qui forment la bande passent continûment d'un caractère à l'autre quand $\mathrm{k}$ augmente. À partir du signe de l'élément de matrice de couplage, on se convainc aisément que pour les états $s$ ( de la bande de conduction), l'état de Bloch de $\mathrm{k}=0$ a l'énergie la plus basse. La situation est opposée pour les liaisons $p-p, \sigma$ de la bande de valence, dont la courbure est négative. Les mêmes résultats se retrouvent en considérant les variations spatiales de la fonction d'onde, qui doivent être minimisées pour abaisser l'énergie. (Cf. fig. 2.3)

2.2.3.3 Les divers états de valence, classés selon leur moment orbital, ont un comportement très différents. Considérons par exemple (figure 2.2) les états $|3 / 2, \pm 3 / 2\rangle$, le moment orbital étant quantifié selon la direction $\mathrm{k} / / \mathrm{z}$. Ils vont donner naissance à une bande relativement étroite (grande masse effective), car le couplage sera pour une bonne part de type $\pi$. Par contre, pour les autres états $|3 / 2, \pm 1 / 2\rangle$ et $|1 / 2, \pm 1 / 2\rangle$, les orbitales $p_{z}$ pointeront en partie les unes vers les autres, introduisant plus de caractère $\sigma$ dans la liaison et une masse effective plus légère. 
Pour une discussion quantitative de ces effets, il vaut mieux considérer la structure zincblende que cubique simple [YC p.78]. Cependant, nous voyons déjà apparaître la distinction entre une bande de trous lourds et deux bandes de trous légers (les deux premières étant dégénérées en $\mathrm{k}=0$ ). Mais nous voyons aussi combien cette classification est dépendante de la direction de $\mathbf{k}$. Une orbitale $\mathrm{X}^{+}$sera "lourde" le long de $\mathrm{k}_{\mathrm{z}}$, en partie légère dans une direction perpendiculaire. Cette remarque n'est pas sans importance dans deux cas que rencontre souvent l'opticien :

- Les cas d'excitation optique à haute énergie au dessus du gap : même dans le cas 3D, les paires électrons trous créées sont réparties dans l'espace des $\mathbf{k}$ en nappes de formes non évidentes, et qui dépendent de la polarisation (Cf. paragraphe 4.2.1).

- Et surtout les structures bidimensionnelles, qui introduisent une direction de croissance privilégiée par rapport à laquelle tout est obligé de s'organiser : le vecteur d'onde, mais aussi le moment cinétique de rotation (Cf. section 3 ).

\subsubsection{Pour aller plus loin}

La portée de la discussion précédente peut-être étendue au-delà de cette approximation "liaison forte et très ionique", dont l'intérêt est plus pédagogique que quantitatif, et ceci dans deux directions indépendantes.

2.2.4.1 Vers les matériaux covalents La structure de bande d'un semi-conducteur élémentaire comme le germanium, de structure "diamant", peut se comprendre comme suit : la maille élémentaire comprend toujours deux atomes par maille, mais identiques. La formation des bandes peut être décomposée en deux temps. D'abord une hybridation $\mathrm{sp} 3$ de type moléculaire, puis l'élargissement en bandes par couplage d'une maille à l'autre. En pratique (YC p.91), les bandes de valence sont issues des orbitales p liantes et la bande de conduction des orbitales s antiliantes, et la "règle de l'octet" prévoit un isolant. Il en est de même pour les semi-conducteurs III-V et II-VI à gap direct les plus usuels (GaAs, CdTe,...), et en pratique les plus utilisés aujourd'hui pour la fabrication d'hétérostructures. Ce sont ces exemples qu'on aura en tête dans la suite de ce cours. Par exemple, les symétries des états de bande, $s$ et $p$ discutées plus haut, avec leurs conséquences qualitatives sur les masses effectives de trou, et aussi sur les règles de sélection des transitions optiques (Cf. parag. 4.2.1), restent des informations pertinentes.

De fait, le caractère covalent de la liaison est prépondérant dans les semi-conducteurs III$\mathrm{V}$ et II-VI, même si l'ionicité prend de l'importance chez ces derniers.

2.2.4.2 Vers les fonctions de Wannier La forme "liaison forte" de la fonction d'onde, de type de l'équation 2.3), présente un intérêt plus général. Elle permet de bien visualiser les situations où la localisation des fonctions d'onde joue un rôle important, par exemple autour d'impuretés ou dans les hétérostructures. En réalité, il est toujours possible d'écrire les fonctions de Bloch sous cette forme agréable, en utilisant la base des fonctions de Wannier :

où :

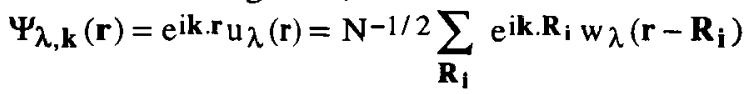




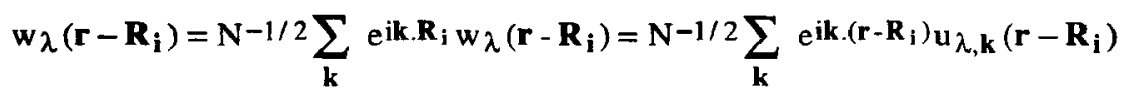

sont les fonctions de Wannier ainsi définies. Les fonctions de Wannier sont labellées par un indice de bande et par un site du réseau réel, elles sont localisées autour de ce site, elles "ressemblent" à des fonctions atomiques, mais sont orthogonales entre sites voisins. Ce sont, en quelque sorte, les pseudo-fonctions atomiques qu'il faut pour penser en liaison forte tout en restant réaliste, puisque les fonctions de Wannier peuvent être construites sur les "vraies" fonctions d'onde de Bloch.

\subsection{Des goûts et des couleurs}

Il n'est pas question ici de prétendre présenter tous les semi-conducteurs imaginables. En réalité, on s'accorde en général à considérer que relèvent de la "physique des semiconducteurs" des familles de composés très étudiées, certains disent trop. C'est un fait qu'avec les succès de ces travaux, sans aucun doute stimulés par les applications, on parvient à une précision de la description peu concevable dans d'autres domaines de la "physique de la matière condensée". Toutes proportions gardées, l'académisme de certains problèmes évoque ce qui a pu se faire en physique atomique. Quelqu'intérêt qu'on y trouve, encore faut-il reconnaître l'accomplissement que cela représente. Mais, rançon du succès, il faut reconnaître que pratiquer cette "physique des semi-conducteurs" nécessite un fond de connaissances préalables assez important pour décourager la curiosité à l'extérieur du domaine. C'est une remarque qui a été faite il y a déjà longtemps par J.J. Hopfield, lors de la Conférence Internationale sur la Physique de Semi-Conducteurs en 1972. Cependant, depuis, les progrès de l'expérimentation et de la technologie ont plusieurs fois fourni des occasions de renouvellement profond, permettant de rétablir le lien avec d'autres domaines de la physique.

Après ces explications, je me sens autorisé à réduire ma présentation aux matériaux semiconducteurs dont, en pratique, il pourra être question dans cette École.

2.3.1 Le tout premier critère de classification est évidemment la nature de la bande interdite ("gap"), direct ou indirect. Dans le premier cas, les états de base de conduction et de valence sont situés "en face" dans l'espace des $k$, et, le plus souvent, en $k=0$. L'absorption optique démarrera à cette énergie franchement, sans besoin de phonon intermédiaire pour satisfaire à la conservation du moment total. C'est donc, réciproquement, la configuration privilégiée des matériaux pour dispositifs émetteurs de lumière, a fortiori pour lasers. Mais les propriétés optiques des matériaux indirects ne sont pas pour autant à négliger. En effet, d'une part il n'y a pas que des dispositifs émetteurs. Par exemple les cellules solaires, les détecteurs. Ensuite, des impuretés profondes ou des microstructures peuvent modifier significativement les propriétés émettrices d'un matériau indirect ( $\mathrm{GaP}$ dopé $\mathrm{N}$, Si poreux). En ce qui concerne le silicium, on comprend que les enjeux industriels induisent une pression formidable pour trouver des dispositifs optiques de fabrication compatible avec sa technologie.

In semble impossible dexpliquer "avec les mains" pourquoi un matériau est direct ou indirect. Il y a l'exemple de la série $\mathrm{GaAs}$ (direct)/ GaP (indirect)/ GaN cubique (direct), bien difficile à comprendre simplement. Autre exemple, le silicium présente son minimum de $\mathrm{BC}$ à $85 \%$ de la ZB dans la direction [001] de l'espace réciproque ; mais dans le germanium, de la même colonne du tableau périodique et qui cristallise de la même façon, ce minimum est situé 
au bord de la ZB dans la direction [111]. La raison en est sans doute que les états de grands $\mathrm{k}$ font intervenir des interférences subtiles entre les influences des seconds voisins. Par contre, on comprendra aisément qu'un extremum de bande se situe volontiers en un point de haute symétrie de la zone de Brillouin, en $\Gamma$ ou, à défaut, en $X$ ou $\mathrm{L}$, avec donc plusieurs vallées dégénérées. Dans ces demiers cas, chacune de ces vallées sera caractérisée par une masse effective très anisotrope.

2.3.2 Le second critère de classification important du point de vue de l'optique est bien entendu la valeur du gap, qui fixe le domaine de longueur d'onde d'intérêt principal du matériau. De plus (Cf. parag. 3.3.2), la valeur du gap direct n'est pas sans relation avec la masse effective de conduction en cet endroit, et donc avec l'énergie de liaison excitonique, une quantité importante pour beaucoup de phénomènes optiques. En pratique, on se préoccupera peu des phénomènes excitoniques dans les semi-conducteurs à petit gap, typiquement inférieur à $0,5 \mathrm{eV}$.

2.3.3 Un troisième critère est l'ionicité. De façon générale, elle accrô̂t la valeur du gap par rapport à la liaison covalente. Elle accroît aussi l'interaction "de Fröhlich" entre électron et phonon optique longitudinal (LO), qui est médiatisé par le champ électrique. Ce n'est pas sans importance pour la relaxation des porteurs [YC p.125].

Ce critère d'ionicité est en partie relié à la "famille" à laquelle se rattache le semiconducteur au regard des colonnes du tableau de Mendeleiev (III-V, II-VI...) Mais l'intérêt de cette classification, la plus utilisée, repose surtout sur les contraintes de la technologie et de la fabrication. D'une part, les problèmes métallurgiques sont voisins au sein d'une même famille ; et aussi, ne l'oublions pas, les composants d'une famille sont en général des dopants pour l'autre, donc actifs à l'état de trace. D'autre part, les possibilités d'ingénierie offertes par les alliages et les hétérostructures s'expriment pour l'essentiel au sein d'une même famille, si l'on veut rester dans le cadre confortable de la "physique des semi-conducteurs", où beaucoup de notions communes restent pertinentes. Cet aspect des choses a pris récemment tant d'importance qu'on parle aujourd'hui de la famille des semi-conducteurs IV-IV, pour évoquer la filière $\mathrm{Si} / \mathrm{Ge} / \mathrm{C}$.

La famille des semi-conducteurs élémentaires comprend certes phosphore, soufre, sélénium et tellure, mais elle est dominée par les éléments $\mathrm{IV}$, le silicium s'étant finalement affirmé hégémonique avec le développement de la microélectronique, probablement pour des raisons technologiques (l'oxyde $\mathrm{SiO}_{2}$ étant un isolant et un passivant extraordinaire) et aussi économiques. Le germanium a été également beaucoup étudié. Tous deux sont restés pendant des décennies des paradigmes de la physique des semi-conducteurs, bien qu'ils soient des matériaux à gap indirect, à une époque où les propriétés optiques étaient plutôt négligées. Ils cristallisent dans la structure "diamant", formée de deux sous-réseaux cubiques à faces centrées (Cf. fig. 2.1). Paradoxalement, dans le domaine des alliages et de l'hétéroépitaxie, la Nature n'a pas été aussi bienveillante pour la filière IV-IV /Si que pour les III-V : les idées furent nombreuses, mais les succès plus rares et, aujourd'hui encore, ces études restent, relativement, peu développées.

Les semi-conducteurs composés III-V ont souvent un gap direct, mais pas toujours, en particulier du côté des grands gaps (AlAs, GaP). Leur structure cristalline est le plus souvent cubique zinc-blende, dérivée donc de la structure "diamant". Ils ont acquis ces dernières 
années une grande importance, car se prêtent bien à l'hétéro-épitaxie et à d'autres opérations technologiques (dopage en particulier) nécessaires pour la réalisation de structures artificielles. Ce sont aujourd'hui par excellence les matériaux du "band-gap engineering", et, en particulier, de l'optoélectronique. Les familles de structures les plus étudiées sont :

- la filière élaborée sur substrat GaAs: GaAs/AlGaAs//nGaAs, intéressante dans la région de longueur d'onde $0.7-0.9 \mu \mathrm{m}$, dont la technologie est toujours la plus avancée, et qui sert de banc d'essai à la plupart des nouvelles idées.

- la filière élaborée sur InP : GaInAs/AlInAs/InP, active dans la fenêtre de longueur d'onde 1.3-1.5 $\mu \mathrm{m}$ qui intéresse les télécommunications.

- les nitrures. Plus récemment des succès spectaculaires et inattendus dans le domaine de l'émission bleue ont relancé l'intérêt pour $\mathrm{GaN}$ et sa famille. Notons que la forme cristalline usuelle de ces matériaux est hexagonale, de type Wurtzite, comme certains matériaux II-VI.

Avec les semi-conducteurs II-VI et I-VI, l'ionicité est de plus en plus marquée ce qui a de nombreuses conséquences :

- métallurgiques, avec en particulier la difficulté de doper, de prendre des contacts.

- structurales, avec beaucoup de composés à grand gap de structure Wurtzite. Elle est finalement assez semblable à la blende de zinc du point de vue des liaisons élémentaires et la physique est assez proche. Cela abaisse la symétrie, la dégénérescence trous lourds-légers en $\mathbf{k}=\mathbf{0}$ est levée, ce qui modifie les règles de sélection sans changer fondamentalement les choses. Il faut de plus noter que les phases cubiques de ces composés sont souvent accessibles aujourd'hui en épitaxie "forcée" sur un substrat cubique (croissance pseudomorphique).

- électroniques et optiques avec beaucoup de grands gaps et des effets excitoniques très marqués.

$\grave{A}$ ces deux familles, on peut adjoindre $\mathrm{CuO}$ et $\mathrm{Cu}_{2} \mathrm{O}$, semi-conducteurs à grand gap bien connus, mais peu remarqués pour leurs perspectives d'application. Notons enfin que les composés II-VI à base de Te, Se, S sont entrés eux aussi, depuis le début des années 80 , dans l'ère de l'hétéroépitaxie et des possibilités d'ingénierie qu'elle offre.

Il faut une mention spéciale pour les composés du mercure. D'une part, la famille des alliages $\mathrm{HgCdTe}$ reste remarquablement accordée en maille sur le substrat CdTe. D'autre part, à cause de la très grande masse du cation, pathologique en quelque sorte, $\mathrm{HgTe}$ est un semiconducteur à gap nul et les alliages fournissent donc une palette de gaps ajustables entre 0 et $1,6 \mathrm{eV}$, avec de grandes possibilités d'application dans le domaine de l'optique infrarouge.

Enfin, citons rapidement quelques composés moins étudiés. Les sels de plomb de type $\mathrm{PbS}$ sont aussi des semi-conducteurs à petit gap, direct, mais situé au point $\mathrm{L}$ de la zone de Brillouin. Les matériaux III-VI, de type GaSe, sont lamellaires, avec donc une grande anisotropie des propriétés électroniques et optiques.

\subsubsection{Les semi-conducteurs magnétiques}

Sans aller trop loin dans l'exotisme, on ne peut oublier de présenter les semi-conducteurs magnétiques. Les plus étudiés ont d'abord été les matériaux de type EuO, aux propriétés magnétiques intéressantes (ferromagnétisme), issues des couches $\mathrm{f}$ de l'europium. Mais une mention spéciale doit être faite dans cette École aux alliages "semimagnétiques" ou magnétiques dilués (DMS) de type CdMnTe. Leurs propriétés magnétiques semblent moins riches, mais l'introduction progressive du magnétisme permet justement de maintenir leur caractère de "bons" semi-conducteurs, propices à des études assez fines. De plus, ils s'insèrent 
bien dans la famille des semi-conducteurs II-VI susceptibles de s'épitaxier les uns sur les autres pour former des hétérostructures alliant des matériaux magnétiques et non magnétiques.
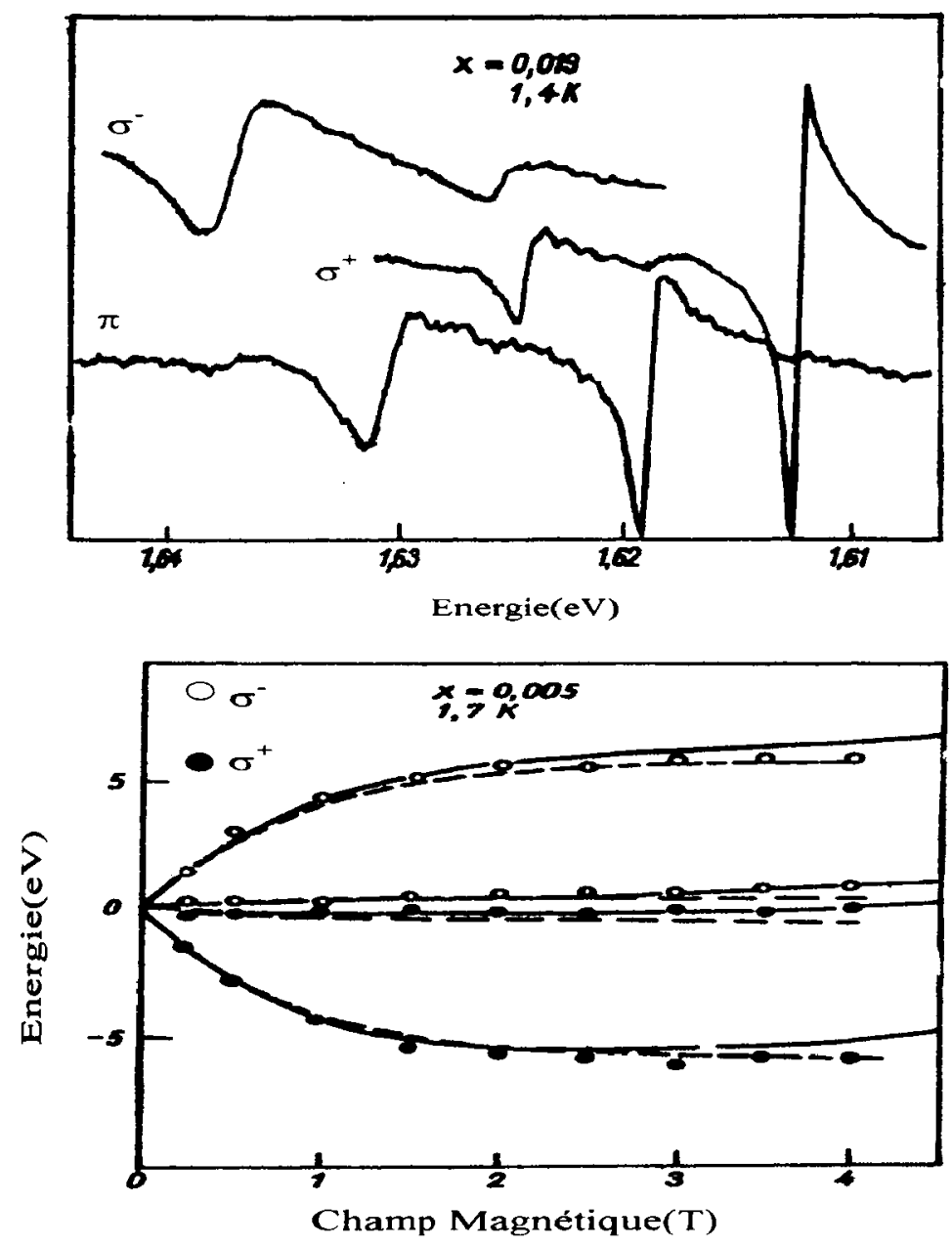

Figure 2.4 : a) Spectres de magnétoréflectivité de CdMnTe pour diverses polarisations en configurations Faraday $\left(H / k_{z}, \sigma^{+}, \sigma^{-}\right)$et Voigt $\left(H \perp k_{z}, \pi\right)$. b) Énergie des transitions en fonction du champ. On reconnaît la loi d'aimantation de Brillouin. (D'après J. Gaj.[12] )

L'essentiel de leurs propriétés physiques peut être compris à l'aide des notions présentées jusqu'ici. Pour rester concret, je m'appuierai sur l'exemple de CdMnTe, dont la réputation et l'utilisation ont débordé le cercle des spécialistes. La structure électronique de l'atome manganèse est $3 \mathrm{~d}^{5}, 4 \mathrm{~s}^{2}$. Du point de vue des couches externes qui participent à la liaison, il peut être considéré comme isoélectronique du cadmium, la couche $\mathrm{d}^{5}$ incomplète et responsable du magnétisme restant pratiquement non perturbée.

Les états délocalisés des bandes moyennent le potentiel désordonné de l'alliage assez raisonnablement pour rester délocalisés et donc satisfaire la loi de Végard (Cf. parag. 3.1.1). 
Le gap $\mathrm{E}_{\mathrm{G}} \mathrm{s}^{\prime}$ accroît, à peu près linéairement, avec la concentration de $\mathrm{Mn}$, et les propriétés du semi-conducteur s'en déduisent sans malice. Dans le cas de $\mathrm{Cd}_{1-\mathrm{x}} \mathrm{Mn}_{\mathrm{x}} \mathrm{Te}$, on sait incorporer jusqu'à $70 \%$ de $\mathrm{Mn}$ avant qu'une transition de phase ne perturbe cette vision simple, et on peut écrire: $\mathrm{E}_{\mathrm{G}}(\mathrm{x})=\mathrm{E}_{\mathrm{G}}(0)+\mathrm{x} \Delta_{\mathrm{G}}(\approx$, pour $\mathrm{CdMnTe}, 1,5 \mathrm{eV}+\mathrm{x} 0,8 \mathrm{eV})$

Quant aux états localisés des couches $d$, ils sont responsables des propriétés magnétiques. En l'occurrence, CdMnTe est paramagnétique avec une forte tendance antiferromagnétique, qui entraîne à très basse température, un comportement de verre de spin. Dans la plupart des cas, l'aimantation s'exprime en fonction du champ magnétique par une loi de Brillouin modifiée de façon phénoménologique : $M=N_{0} \times S_{0} B_{5 / 2}\left[H /\left(T+T_{A F}\right)\right]$ où :

- $N_{0} \times$ est la concentration volumique de $\mathrm{Mn}$,

- $\mathrm{S}_{0}$ est la valeur effective du spin associée à chaque atome $\mathrm{Mn}$; aux faibles concentrations, elle vaut $5 / 2$, la valeur de l'atome isolé ; elle diminue avec la concentration, rendant compte en partie de la tendance antiferromagnétique dans ce milieu désordonné.

- $\mathrm{B}_{5 / 2}(\mathrm{H} / \mathrm{T})$ est la loi de Brillouin qui décrit le paramagnétisme d'un ensemble de moment $5 / 2$ indépendants [11]. La température $\mathrm{T}_{\mathrm{AF}}$, positive, complète la description de la tendance antiferromagnétique

L'originalité de ces matériaux apparaît quand on en vient aux propriétés magnétooptiques. Celles-ci se caractérisent par un clivage (splitting) de spin des états de conduction et de valence proportionnel à l'aimantation et vite gigantesque, de l'ordre de plusieurs dizaines de $\mathrm{meV}$ par tesla. Souvent, il est légitime de négliger les autres effets associés au champ magnétique, effet Zeeman normal, et quantification de Landau. Cet effet, appelé un peu improprement "Zeeman géant", est da à l'interaction d'échange entre états d d'une part, $s$ ou $p$ d'autre part. On peut représenter cette interaction par un hamiltonien de type Heisenberg :

$\mathrm{H}_{\mathrm{ex}}=\sum_{\mathrm{i}}-\mathrm{J}(\mathrm{r}-\mathrm{Ri}) \sigma . \mathrm{S}_{\mathrm{i}}$, où $\mathbf{r}$ et $\sigma$ sont les opérateurs position et spin de l'électron (délocalisé) des bandes, et $\mathbf{R}_{\mathbf{i}}$ et $\mathbf{S}$ ceux des couches d (localisées) des ions $\mathbf{M n}$. J est l'opérateur d'échange s-d ou p-d, selon la bande concernée. Il s'en suit que les extrema de bande sont clivés selon :

$\Delta_{\mathrm{c}}=\mathrm{N}_{0} \alpha \times\left\langle\mathrm{S}_{\mathrm{Mn}}\right\rangle \sigma_{\mathrm{c}}$ pour la bande de conduction, et

$\Delta_{\mathrm{v}}=\mathrm{N}_{0} \beta \times\left\langle\mathrm{S}_{\mathrm{Mn}}\right\rangle \sigma_{\mathrm{v}}$ pour la bande de valence,

où $\mathrm{N}_{0}$ est la densité de site cation, et $\alpha$ et $\beta$ sont respectivement les intégrales d'échange s-d et p-d sur la maille unité, de type $\langle\Psi|J| \Psi\rangle$. Dans une théorie de champ moyen tout à fait légitimée par l'expérience, leur valeur est indépendante de la concentration. Elles sont en pratique proches d'un matériau à l'autre.

Pour CdMnTe, $\mathrm{N}_{0} \alpha=0,22 \mathrm{eV}$ et $\mathrm{N}_{0} \beta=-0,88 \mathrm{eV}$. Il est remarquable que le signe de l'échange est opposé pour les deux bandes. $\left\langle\mathrm{S}_{\mathrm{Mn}}>\right.$ est l'aimantation exprimée en valeur moyenne du spin des ions $\mathrm{Mn}$, au maximum $5 / 2$.

$\sigma_{\mathrm{c}}\left(\right.$ et $\sigma_{\mathrm{v}}$ ) est la valeur moyenne du spin dans l'état considéré, et non pas la valeur (propre) du moment orbital. Ces deux quantités sont, dans une structure cubique, strictement proportionnelles, comme on peut s'en convaincre sur les fonctions de la figure 2.2. Il n'en va pas toujours de même, et il faudra être prudent, dans un matériau hexagonal ou une hétérostructure.

De ce clivage de spin résultent bien sûr des propriétés magnéto-optiques géantes. En effet, à chaque couple d'états de valence et conduction, états propres du moment orbital, sont associées des transitions optiques sélectionnées en polarisation, comme on l'expliquera plus en détail dans la section 4, et comme l'illustrent les résultats expérimentaux de la figure 2.4 . 
Ces différences d'énergie entre transitions optiques de polarisations différentes entraînent un dichrö̈sme et une biréfringence circulaires induits (effet Faraday) eux aussi "géants".

\section{LES HÉTÉROSTRUCTURES À BASE DE SEMI-CONDUCTEURS}

Malgré quelques travaux précurseurs de grande importance, on peut considérer que c'est à la fin des années 70 qu'a vraiment éclaté la révolution des hétérostructures artificielles fabriquées à base de semi-conducteurs. Elle a apporté un renouvellement des thématiques, la multiplication des possibilités et de nouvelles perspectives d'applications. Cela a été possible grâce aux progrès de la technologie, et d'abord de l'épitaxie, et a beaucoup modifié l'exercice du métier de chercheur: il fallut travailler plus à la manière d'un ingénieur, dans des laboratoires richement dotés mais sachant regrouper des équipes pluri-disciplinaires.

Deux livres, pour qui voudra approfondir la physique des hétérostructures bidimensionnelles, restent un bon fondement malgré leur relative ancienneté. En effet, ils ont été écrits après la période la plus féconde du début des années 80. Il s'agit du livre de Bastard [13], surtout intéressant pour regrouper de nombreux résultats théoriques, et du cours de l'École des Houches de 1985 [14], qui présente un spectre plus large de sujets (matériaux contraints, phonons, propriétés de transport...)

\subsection{Les fondements}

\subsubsection{Les alliages}

Cette révolution repose d'abord sur les "bonnes" propriétés de beaucoup d'alliages ; il s'agit bien entendu d'alliages semi-conducteurs unissant deux matériaux de la même famille. Le paradigme en est $\mathrm{Ga}_{1-\mathrm{X}} \mathrm{Al}_{\mathrm{X}} \mathrm{As}$, où $\mathrm{Ga}$ et $\mathrm{Al}$ sont isoélectroniques. Je noterai souvent GaAlAs lorsqu'il n'y a pas d'ambiguité. Parmi les plus populaires, il faut citer aussi GaInAs, AlInAs et même les

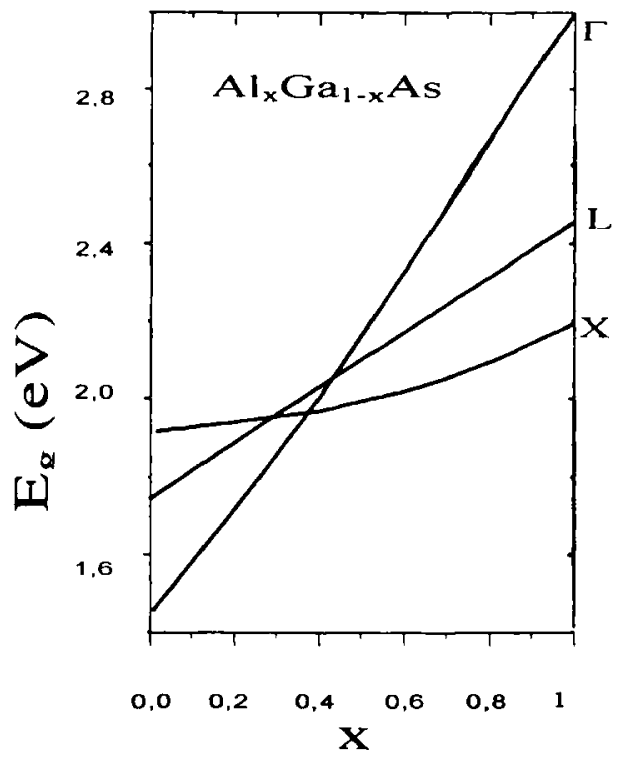

Figure 3.1 : Variation de l'énergie des minima $\Gamma, X$ et $L$ pour l'alliage désordonné $\mathrm{Al}_{\mathrm{x}} \mathrm{Ga}_{1-\mathrm{x}} \mathrm{As}$ en fonction de $\mathrm{x}$, à $\mathrm{T}=300 \mathrm{~K}$. L'origine des énergies est prise au sommet de la bande de valence. L'intersection des courbes associées à $\Gamma$ et $X$ est située vers $x=0.4$. On remarque un écart à la loi linéaire de Végard, appelé "bowing". quaternaires GaAlInAs, accordés en maille sur InP (utilisés pour l'optique à 1,3-1,5 $\mu \mathrm{m}$ des télécommunications), $\mathrm{CdZnTe,} \mathrm{(composant} \mathrm{de}$ base des hétérostructures II-VI), CdMnTe (magnétique dilué), $\mathrm{HgCdTe}$ (détecteurs infrarouge), CdSSe (filtres optiques dans la région du visible), SiGe (permettant d'introduire des hétérojonctions dans l'électronique silicium). Au premier ordre, et s'il n'y a pas de transition de phase cristalline, ils se comportent comme des matériaux de propriétés moyennes, interpolées linéairement entre celles des deux parents : c'est la loi de Végard, qui concerne les paramètres de maille, mais aussi les énergies des bandes et tout ce qui en découle plus ou moins (masses effectives, constante diélectrique...). Il faut cependant prendre la 
précaution de comparer ce qui est comparable, c'est-à-dire les propriétés des bandes aux mêmes points de la zone de Brillouin. On le voit sur la figure 3.1 dans le cas de GaAlAs.

Cette loi, lorsqu'elle s'applique aux propriétés électroniques implique que les états soient bien délocalisés et moyennent les irrégularités du potentiel, les deux aspects étant reliés intimement. Alors, les effets d'alliages pourront être soit négligés, soit traités en perturbation. Par exemple, on introduira une contribution supplémentaire à la diffusion des porteurs, ou une largeur en énergie et en $\mathrm{k}$ des courbes de dispersion. Ou bien encore, on raisonnera à partir d'états localisés, sur des distances grandes par rapport à la maille élémentaire ; cela permet de les construire à partir des états délocalisés, dans l'esprit de l'EFA (Cf. parag. 3.1.4).

Il faut cependant savoir qu'une foi aveugle en la loi de Végard peut apporter de grosses surprises. D'une part, certains alliages sont le siège de phénomènes de démixtion des atomes qui conduisent à un ordre spontané; c'est observé, par exemple, avec l'alliage GaInP, intéressant car épitaxiable sur GaAs sans contenir d'aluminium. D'autre part, certaines substitutions d'éléments isoélectroniques ne se passent pas "bien". C'est le cas, connu depuis longtemps, de l'azote dans $\mathrm{GaP}$, qui forme, à l'état d'impureté, un piège très profond pour les électrons de conduction. La localisation se fait sur une distance qui n'est pas grande par rapport à la maille ; en conséquence, l'état lié mélange des états de toute la zone de Brillouin, ce qui d'ailleurs confêre à ce matériau indirect de bonnes propriétés d'émetteur de lumière. Pour des raisons sans doute similaires, les alliages GaAsN, qui sont étudiés depuis peu, comportent des déviations considérables à la linéarité : en partant de GaAs (gap $=1.4 \mathrm{eV}$ ), le gap de GaAsN commence par diminuer jusque, semble-t-il, moins de $1 \mathrm{eV}$ avant, probablement, de remonter vers celui de $\mathrm{GaN}(3 \mathrm{eV})$.

\subsubsection{L'épitaxie.}

Un autre prérequis pour la physique des hétérostructures est la possibilité de réaliser de bonnes épitaxies. L'épitaxie idéale pourrait être considérée comme une vue de l'esprit, mais c'est un fait que la Nature nous la laisse entrevoir. Elle repose sur la continuité du réseau cristallin au travers de l'interface, celle-ci étant aussi abrupte et plane que possible. Comme pour les alliages, on comprend que dans la plupart des cas, l'épitaxie allie des matériaux de la même famille et aussi de même structure cristalline (le plus souvent cubique zinc-blende). Pour l'ingénieur en structure de bande, des tableaux tels celui de la figure 3.2 sont une source d'inspiration. Source partielle, car il est difficile d'y faire figurer toutes les informations de la structure de bande, gap direct ou indirect, décalage de bande entre les divers matériaux...

La continuité du réseau ne peut être maintenue sur de grandes épaisseurs que si les matériaux ont à très peu près $(<1 / 1000)$ les mêmes paramètres cristallins naturels, c'est-à-dire à l'état massif. C'est le cas pour GaAs, AlAs et leurs alliages et, dans une moindre mesure, pour HgTe, CdTe et leurs alliages. Mais l'utilisation d'alliages de concentration judicieusement déterminée permet de contourner cette difficulté ; les meilleurs exemples en sont $\mathrm{Ga}_{0.47} \mathrm{In}_{0.53}$ As et $\mathrm{Al}_{0.48} \mathrm{In}_{0.52}$ As, tous deux accordés sur InP.

Lorsque le désaccord de maille reste, typiquement, inférieur à $1 \%$, il est souvent possible de faire croître des couches peu épaisses, des puits quantiques par exemple, dont la structure se déforme élastiquement sous l'effet de la contrainte pour adapter les paramètres de maille dans le plan. On parle de croissance pseudo-morphique, la structure pouvant rester d'excellente qualité, avec une densité limitée de défauts ponctuels ou étendus. II n'en est plus de même au-delà d'une épaisseur critique, où la couche minimise son énergie élastique, relaxe plastiquement, en émettant des dislocations. Cette épaisseur critique est, grosso modo, 


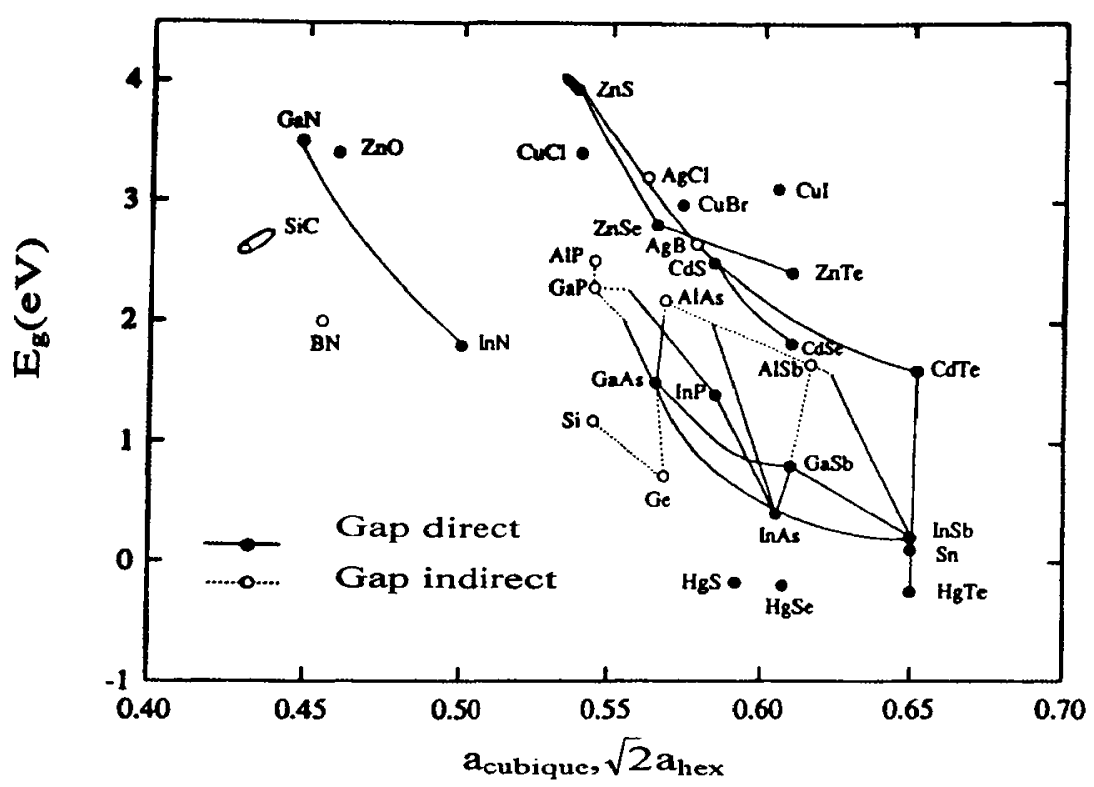

Figure 3.2 : Largeur du gap en fonction du paramètre de maille (cubique ou hexagonale) pour divers matériaux et alliages. N'apparaissent pas sur ce genre de schéma des quantités intéressantes comme les décalages de bandes. (Emprunté à C.F.Klingshirn [5] p.147)

inversement proportionnelle à la densité d'énergie élastique stockée, donc au désaccord. Mais comme la génération de dislocation nécessite le franchissement de barrières d'énergie, elle peut être dépassée dans certaines conditions de croissance, laissant la couche contrainte en équilibre métastable. Au contraire, une fois relaxée, la couche contient une densité élevée de dislocations, souvent incompatible avec de bonnes propriétés électroniques et optiques.

Ajoutons ici que le terme pseudo-morphique et les considérations ci-dessus peuvent s'appliquer aussi à d'autres modes de croissances forcées par le substrat, comme des structures cubiques pour un matériau naturellement hexagonal.

Au-delà d'un désaccord de l'ordre du pour cent, on entre dans le domaine des croissances exotiques, souvent difficilement prévisibles, mais qui peuvent révéler de bonnes surprises. Par exemple, dans le système InAs/GaAs, caractérisé par un désaccord de maille de $7 \%$, la croissance s'effectue en îlots, sans génération de dislocations. Leur taille est encore imparfaitement contrôlée, mais ce sont de bons exemples de "boîtes quantiques".

Ne nous y trompons pas. Aucune technologie n'est parfaite. L'épitaxie GaAs/GaAlAs a été bien sûr l'objet des études les plus détaillées pour apprécier concrètement les écarts à l'idéalité de la structure. Les résultats dépendent en grande partie de l'expérience en cause. Nous sommes par excellence dans le domaine quantique, où il ne peut être question d'isoler la connaissance de l'objet de la nature de la sonde. Autrement dit, même la caractérisation des structures peut mettre en jeu les techniques les plus sophistiquées

Une bonne façon d'appréhender cette réalité est de faire l'historique des études sur la rugosité et la gradualité des interfaces dans ce système école. Depuis la fin des années 70 , on sait par des études de photoluminescence que la hauteur moyenne des rugosités d'interface est d'une monocouche. En effet la raie de recombinaison d'exciton était d'une largeur variable avec l'épaisseur du puits, correspondant à l'incertitude introduite par de telles fluctuations de 
l'épaisseur [15]. Ceci renforcait l'idée, acquise par les caractérisations in situ de diffraction d'électrons par la surface, d'une croissance EJM bidimensionnelle, couche par couche. Puis, on observa [16] non pas une raie large, mais plusieurs raies fines, dont l'écart en énergie correspondait à une monocouche. Il en fut déduit que la taille latérale des îlots d'épaisseur pouvait varier grandement en fonction des conditions de croissance :

- d'une situation "lisse", aux très grands îlots presque découplés électroniquement,

- à une situation "rugueuse", où la taille des îlots est comparable à la longueur des cohérence des excitons.

- et à une situation "pseudo-lisse", à très fine rugosité moyennée comme un alliage.

La longueur de cohérence des excitons a rapidement été assimilée au rayon de Bohr, alors qu'il s'agit d'une notion qui affecte le mouvement d'ensemble de l'exciton, ou de tout autre particule (électron, phonon...). Cette erreur est aujourd'hui ancrée dans le vocabulaire courant, mais a été relevée et a suscité des analyses théoriques du phénomène nettement plus solides [17].

Plus tard, a été mis en évidence [18] le rôle de la gradualité, par le confinement des phonons LO, et qui semble avoir pour origine principale l'échange des atomes dans les deux premières monocouches du front de croissance. Une bonne synthèse de ces questions a pu être réalisée vers le début des années 90 [19], jusqu'à ce que l'avènement récent des techniques de microluminescence [20] permettent d'approfondir, ou de compliquer encore les choses.

En résumé, l'épitaxie de couches bidimensionnelles a joué un rôle moteur dans la révolution des hétérostructures, et continue de fournir une majorité des structures pour l'optique. Cependant, il faut mentionner les autres technologies qui l'accompagnent.

\subsubsection{Les autres technologies.}

Ce sont d'abord les techniques de la microfabrication et, en particulier, de la microélectronique : lithographie optique et électronique, masquage, implantation localisée, gravure chimique ("humide") et ionique ("sèche"). Au laboratoire, elles viennent souvent, mais pas exclusivement, en complément de l'épitaxie pour définir des structures dans le plan, tels que motifs pour les expériences de transport électronique, dispositifs électro-optiques, détecteurs, modulateurs ou émetteurs de lumière, fils et boîtes quantiques. Elles sont souvent assez lourdes, mais relativement contrôlables. Elles sont bien adaptées aux Sciences de l'Ingénieur.

Ensuite, il faut mentionner les usages extrêmes de l'épitaxie pour réaliser des structures de dimensionnalité inférieure à 2 . C'est le cas des îlots InAs/GaAs déjà cités, où on utilise astucieusement les tendances naturelles au facettage de la croissance. On parle de croissance "auto-organisée". C'est aussi le cas de la croissance sur surface "vicinale", légèrement désorientée par rapport à un plan principal du réseau. Une telle surface comporte un réseau de marches monoatomiques qui fourniront des sites de nucléation privilégiée aux "adatomes" déposés pendant l'épitaxie. On parvient ainsi, en modulant la nature des "adatomes", à abaisser la symétrie bidimensionnelle de la couche. Dans cette catégorie, on peut aussi ranger la croissance dans des sillons en $\mathrm{V}$ préalablement gravés de façon adéquate. On parvient, en jouant sur les différentes propriétés de facettage de GaAs et AlAs, à réaliser des fils quantiques.

Enfin, n'oublions pas tout un ensemble de techniques variées, souvent modestes et astucieuses, qui permettent de réaliser de très beaux objets. On peut citer la manipulation d'atomes par des techniques de microscopie à pointe, et aussi, plus utiles dans le domaine de 
l'optique, le remplissage des zéolithes ou des opales. II y a aussi l'exemple des techniques solgel qui permettent de réaliser des nanocristaux noyés dans une matrice transparente.

\subsubsection{La théorie EFA (Approximation de la Fonction Enveloppe).}

C'est un peu par provocation que je cite une théorie parmi les fondements qui ont rendu possible le développement des hétérostructures. Bien entendu, c'est une théorie limitée, nécessairement approximative, parmi d'autres plus fondamentales, plus proches des premiers principes. Mais elle a d'abord le mérite d'être intuitive et utilisable quantitativement par tout expérimentateur. Ensuite elle fournit dans la plupart des cas des résultats numériques de précision suffisante, nous verrons plus loin pourquoi. C'est donc par excellence l'outil de " l'Ingénieur en Structure de Bande », car, en ce domaine, il n'y a guère eu de réalisation sans prévision.

Il est utile dès maintenant d'en présenter le principe sur le cas simple d'une bande présentant un extremum non dégénéré en $\mathrm{k}=0$, par exemple la bande de conduction de GaAs et de $\mathrm{Ga}_{0,7} \mathrm{Al}_{0,3} \mathrm{As}$. Nous avons vu (éq.2.1) que la forme générale des fonctions d'onde (de Bloch) du cristal infini était :

$$
\Psi_{\lambda, \mathbf{k}}(\mathbf{r})=\mathrm{V}-1 / 2 \mathrm{e}^{\mathrm{ik.r} \mathbf{r}} \mathrm{u}_{\lambda, \mathbf{k}}(\mathbf{r})
$$

où $\lambda$ est l'indice de l'extremum et où $u_{\lambda, k}(r)$ est périodique, comme le potentiel du cristal, donc rapidement variable à l'échelle de la maille élémentaire. Dans la région de l'espace des $\mathbf{k}$ proche de l'extremum $k=0$, il est raisonnable de supposer que les fonctions $u_{\lambda, k}(r)$ ne dépendent pas de $\mathbf{k}: \mathbf{u}_{\lambda, \mathbf{k}}(\mathbf{r})=\mathbf{u}_{\lambda, 0}(\mathbf{r})$. De plus, l'exponentielle $\mathrm{e}^{\mathrm{ik.r}}$, appelée fonction enveloppe, est alors lentement variable, et l'énergie des états suit une loi parabolique de masse effective $\mathrm{E}=\hbar^{2} \mathrm{k}^{2} / 2 \mathrm{~m}$. On peut donc considérer que tout se passe comme si l'électron se déplaçait dans l'espace libre, avec une fonction enveloppe qui joue le rôle de sa fonction d'onde, à deux détails d'importance près : la masse effective $m$, et l'énergie potentielle, égale à l'énergie de l'extremum.

Dans une hétérostructure, par exemple un puits quantique bidimensionnel de largeur $\mathrm{L}$ formé du matériau $A$ entre deux barrières du matériau B, l'approximation de la fonction enveloppe consiste à écrire les fonctions d'onde sous la forme :

$$
\Psi_{\lambda, n}(\mathbf{r})=F_{\lambda, n}(\mathbf{r}) u_{\lambda, 0}(\mathbf{r})
$$

où $F_{\lambda, n}(r)$ est lentement variable, et $n$ représente l'ensemble des nombres quantiques qui labellent l'état, comme le faisait le vecteur d'onde dans le matériau massif. Cette fonction enveloppe est fonction propre du hamiltonien effectif $H(r)=-\hbar^{2} \nabla^{2} / 2 m_{\text {eff. }}(z)+V_{\text {eff. }}(z)$, avec une énergie potentielle et une masse effective dépendant de $z$, la direction de croissance. La signification de cette forme de fonction d'onde est illustrée par la figure 3.3. Ceci repose sur l'hypothèse, forte, que les fonctions rapidement variables sont non seulement indépendantes de $\mathrm{k}$, mais aussi les mêmes dans les deux matériaux :

$$
u_{\lambda, 0}^{A}(\mathbf{r}) \approx u_{\lambda, 0}^{B}(r)
$$

Une autre approche pour introduire l'EFA, parfaitement complémentaire, est encore plus parlante. Elle repose sur l'écriture de la fonction d'onde sur la base des fonctions de Wannier (Cf. paragraphe 2.2.4.2) : 


$$
\begin{aligned}
& \Psi_{\lambda, \mathbf{n}}(\mathbf{r})=\mathrm{N}^{-1 / 2} \sum_{\mathbf{R}_{\mathbf{i}}} F_{\lambda, \mathrm{n}}\left(\mathbf{R}_{\mathbf{i}}\right) w_{\lambda}^{\mathrm{A}}\left(\mathbf{r}-\mathbf{R}_{\mathbf{i}}\right) \text { dans le matériau A } \\
& \Psi_{\lambda, \mathrm{n}}(\mathbf{r})=\mathrm{N}^{-1 / 2} \sum_{\mathbf{R}_{\mathbf{i}}} \mathrm{F}_{\lambda, \mathbf{n}}\left(\mathbf{R}_{\mathbf{i}}\right) w_{\lambda}^{\mathrm{B}}\left(\mathbf{r}-\mathbf{R}_{\mathbf{i}}\right) \text { dans le matériau B }
\end{aligned}
$$

et la fonction enveloppe $F_{\lambda, n}(R)$ sera cette fois la fonction continue de $\mathbf{R}$, qui reproduit, en la lissant, la variation des coefficients $\mathbf{F}\left(\mathbf{R}_{\mathbf{j}}\right)$. Mais il faut supposer que les fonctions de Wannier restent une bonne base, donc restent orthogonales en passant d'un matériau à l'autre. Cette condition est équivalente à (3.2) qui affecte les fonctions de Bloch.

Etat délocalisé dans le réseau massif:

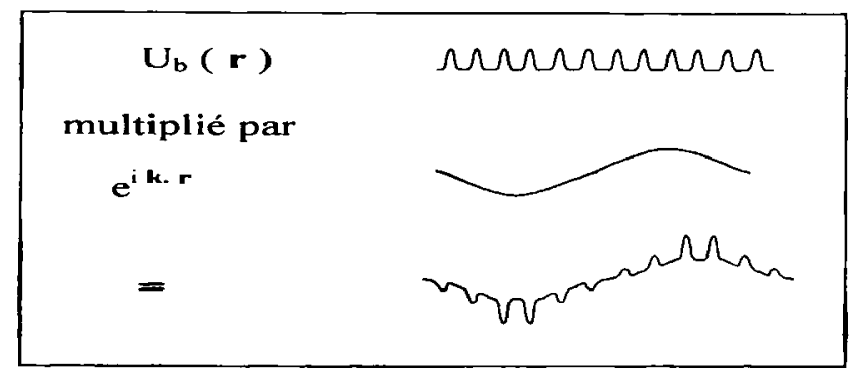

Etat localisé dans un puits quantique:

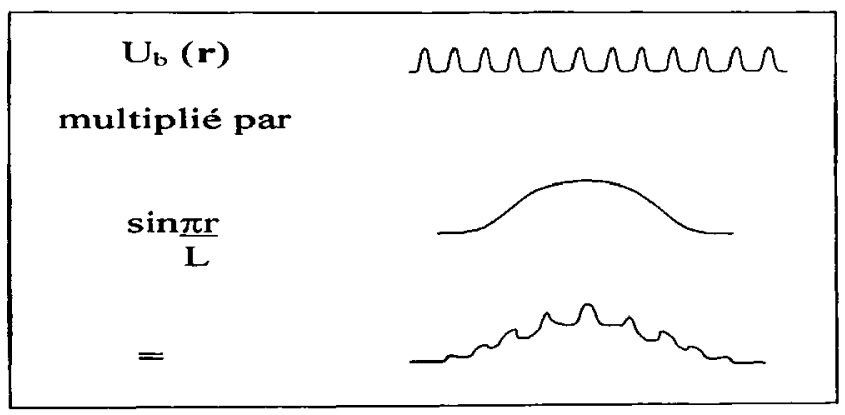

Figure 3.3 : L'esprit de l'Approximation de la Fonction Enveloppe

En tout cas, quelle que soit la façon dont l'EFA est justifiée, on en vient aisément au modèle de Ben Daniel-Duke : la fonction enveloppe est fonction propre de l'équation de Schrödinger suivante :

$$
\left[-\frac{\hbar^{2}}{2} \frac{\partial}{\partial z} \frac{1}{m(z)} \frac{\partial}{\partial z}-\frac{\hbar^{2}}{2 m_{\perp}(z)}\left(\frac{\partial^{2}}{\partial x^{2}}+\frac{\partial^{2}}{\partial y^{2}}\right)+V(z)\right] F(\mathbf{r})=\varepsilon F(\mathbf{r})
$$

où il faut remarquer que la masse effective dépendant de $\mathrm{z}$, l'énergie cinétique est écrite de façon à respecter l'hermiticité du hamiltonien. De plus, on n'exclut pas la possibilité que la 
masse effective pour le mouvement dans le plan $\mathrm{m}_{\perp}$ soit différente de la masse de confinement $\mathbf{m}$. C'est souvent une situation à prendre en compte pour les états de valence (Cf. parag. 2.2.3.3).

Les variables $x, y$ et $z$ se découplent sans difficulté, et la particule est libre dans le plan :

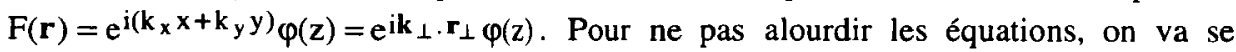
restreindre au cas de deux matériaux $\mathbf{A}$ et $\mathbf{B}$ formant, par exemple, un puits quantique. La condition de continuité de la fonction d'onde aux interfaces impose évidemment que $\mathbf{k}_{\perp}$ est le même dans les matériaux A et $\mathrm{B}$. II vient donc pour le mouvement en $\mathrm{z}$ :

$$
\left[-\frac{\hbar^{2}}{2 m^{(A \text { ou B })}} \frac{\partial^{2}}{\partial z^{2}}-\frac{\hbar^{2}}{\left.2 m_{\perp}^{(A ~ o u ~ B ~}\right)} k_{\perp}^{2}+V(A \text { ou B })\right] \varphi^{(A \text { ou B })}(\mathrm{z})=\varepsilon \varphi(\text { A ou B })(z)
$$

On se trouve face à un problème de mécanique quantique relativement classique. Intéressons-nous aux états liés du puits quantique. Dans le matériau A (puits) l'énergie cinétique en $z$ est positive, c'est-à-dire $: T_{Z}^{A}=E-V-\hbar^{2} k_{\perp}^{2} / 2 m_{\perp}^{A}>0$. Donc les solutions sont des ondes planes de vecteur d'onde $\pm \kappa=\hbar-1 \sqrt{\mathrm{T}_{\mathrm{Z}}^{\mathrm{A}} / \mathrm{m}^{\mathrm{A}}}$ :

$$
\varphi^{A}=a_{1} e^{i k z}+a_{2} e^{-i k z}
$$

Par contre, dans les régions $\mathrm{B}$ (Barrières) où $\mathrm{T}_{\mathrm{Z}}^{\mathrm{B}}<0$, ce sont des exponentielles réelles de paramètre $\pm \tau=\hbar^{-1} \sqrt{-T_{\mathrm{Z}}^{\mathrm{B}} / \mathrm{m}^{\mathrm{B}}}$ :

$$
\varphi^{\mathrm{B}}=\mathrm{b}_{1} \mathrm{e}^{\tau z}+\mathrm{b}_{2} \mathrm{e}^{-\tau z}
$$

Pour déterminer les fonctions et valeurs propres, on utilise également les conditions aux limites :

- à l'infini, ondes évanescentes, donc $b_{1}(z \longrightarrow-\infty)=0$ et $b_{2}(z \longrightarrow+\infty)=0$

- aux interfaces, notées $z=i$, continuité de la fonction enveloppe et, non pas simplement de sa dérivée, mais du courant $\operatorname{Im}\left(\varphi^{*} \frac{\hbar}{\mathrm{m}} \frac{\partial \varphi}{\partial z}\right)[21]$. Ceci impose :

$$
\begin{gathered}
\varphi^{\mathrm{A}\left(\mathrm{z}=\mathrm{i}^{+}\right)}=\varphi^{\mathrm{B}}(\mathrm{z}=\mathrm{i}-) \\
\frac{1}{\mathrm{~m}^{\mathrm{A}}} \frac{\partial \varphi^{\mathrm{A}}\left(\mathrm{z}=\mathrm{i}^{+}\right)}{\partial \mathrm{z}}=\frac{1}{\mathrm{~m}^{\mathrm{B}}} \frac{\partial \varphi^{\mathrm{B}}(\mathrm{z}=\mathrm{i}-)}{\partial \mathrm{z}}
\end{gathered}
$$

Dans le cas de hauteur de barrière $V_{B}$ infinie, la fonction d'onde doit simplement s'annuler à l'interface, et on retrouve sans difficulté, puisque le potentiel est symétrique, que les solutions sont des cosinus (symétriques) ou des sinus (antisymétriques). La tradition s'est établie de numéroter ces états à partir de 1 . Plus précisément :

$$
\begin{aligned}
& \varphi_{2 p+1}(z)=(2 / L)^{1 / 2} \cos [(2 p+1) \pi z / L] \\
& \varphi_{2 p+2}(z)=(2 / L)^{1 / 2} \sin [(2 p+2) \pi z / L]
\end{aligned}
$$

d'énergie $\frac{\hbar^{2}}{2 \mathrm{~mA}^{\mathrm{A}}}\left[\begin{array}{c}(2 \mathrm{p}+1) \\ \text { ou }(2 \mathrm{p}+2)\end{array}\right]^{2}+\mathrm{VA}$ 
avec pentier $\geq 0$ et $\mathrm{z} \leq \mathrm{L} / 2$.

Mais en général, la résolution de ces équations ne peut être que numérique. À ce prix, il est raisonnable d'y inclure aussi les effets de non-parabolicité dans la bande de conduction. Par ailleurs, on comprend que le confinement de la bande de valence dégénérée en $K=0$ introduise une situation physique plus complexe, et nécessite un traitement particulier. Je traiterai donc tout cela globalement au paragraphe 3.3 .

On trouvera dans le livre de Yu et Cardona [YC p.152], une présentation plus développée et plus rigoureuse de l'EFA, fondée sur les combinaisons linéaires de fonctions localisées de Wannier. Dans le livre de Bastard [13], elle est présentée d'un point de vue très complémentaire, comme une extension de la théorie k.p de Kane aux hétérostructures.

Discutons maintenant de l'intérêt et des limites de la théorie EFA.

- Comme on le voit, elle se nourrit de paramètres de masse effective qu'il est possible d'ajuster. Cela permet, avec quelques échantillons bien choisis, de "calibrer" sa théorie pour toute une famille de structures.

- Sa principale condition de validité est que le potentiel effectif varie lentement à l'échelle de la maille élémentaire. C'est du moins ce qu'on doit supposer pour la justifier rigoureusement. Cependant, son efficacité pour prévoir les énergies de super-réseaux à très courte période a étonné plus d'un expérimentateur. En réalité, il apparaît que la condition de variation lente porte sur la fonction enveloppe elle-même, ce qui peut être beaucoup moins sévère. On retrouve ici une situation physique analogue à celle de la loi de Végard pour les "bons" alliages (Cf. parag. 3.1.1).

- En pratique, sa validité est donc rarement mise en défaut, d'autant moins qu'aux incertitudes expérimentales (résolution d'une expérience de spectroscopie), il faut ajouter les incertitudes technologiques (largeur du puits, raideur des interfaces), ou celles sur les paramètres utilisés.

- La condition (3.2) est évidemment essentielle. En particulier, la théorie EFA ne peut associer, dans les deux matériaux hôtes, que des fonctions d'ondes de même symétrie pour construire celles de l'hétérostructure. Le cas du système GaAs/AlAs est, de ce point de vue, très instructif (Cf. parag. 3.2.2).

\subsection{Zoologie des micro- et nanostructures}

Dans les années 80 , les techniques d'épitaxie n'ont cessé de progresser et d'étendre la physique des hétérostructures bidimensionnelles à d'autres systèmes que GaAs/GaAlAs. Aujourd'hui, beaucoup sont accessibles couramment, et disponibles pour des études variées avec une bonne reproductibilité. Il est donc apparu indispensable dans cette École de présenter en détail les structures bidimensionnelles. De plus, cela nous permettra d'expliciter des notions généralisables aux structures de dimensionnalité inférieure. Les structures à O-Dimension seront elles aussi présentées en détail à travers l'exemple des nanocristaux II-VI et I-VII (Cf le cours de D.Ricard).

On va donc tenter de brosser à grands traits un bilan sommaire, et provisoire, des efforts faits pour concevoir et fabriquer des hétérostructures à base de semi-conducteurs pour l'optique. 
(a)

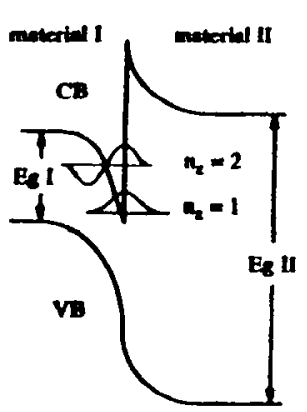

(d) (b)

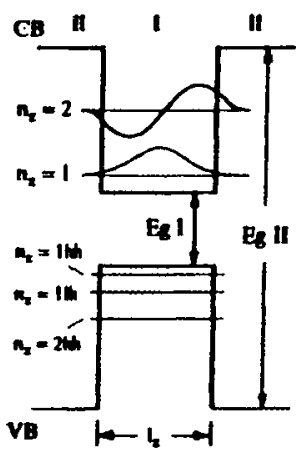

(e) (c)

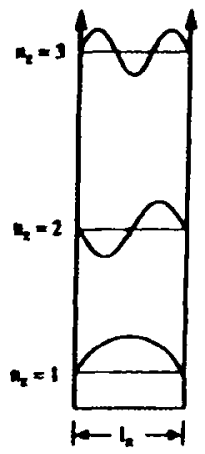

(f)

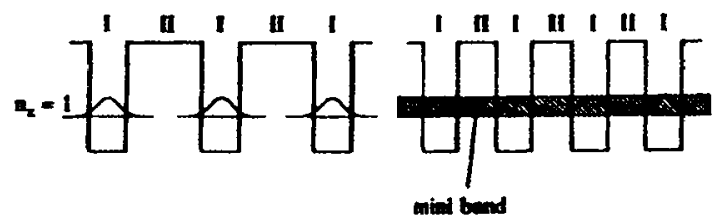

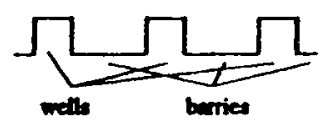

(s) (b)

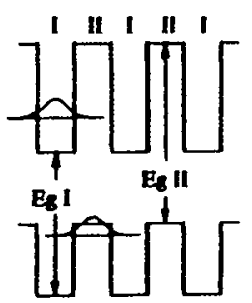

(I)
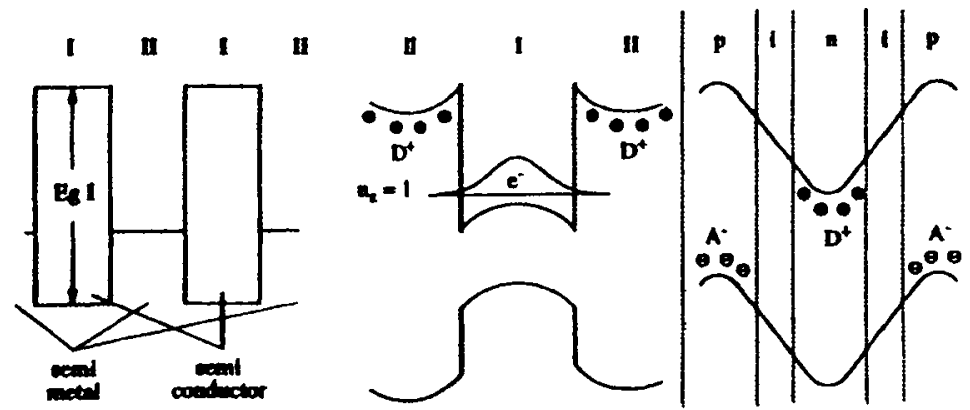

Figure 3.4 : Zoologie des hétérostructures 2D, vue par C.F.Klingshirn [5] p.145 : a) Hétérojonction à dopage modulé. La courbure de bande est provoquée par la charge d'espace des donneurs dont les électrons sont transférés dans le canal. b) Puits quantique et ses niveaux confinés. c) Puits quantique idéal de barrière infinie.

d) Multipuits quantique de type I. e) Super-réseaux de type I, dont les états de conduction sont délocalisés.

f) Super-réseau de type II. g) Super-réseau de type III. h) Puits quantique dopé par transfert de charge. i) Superréseau obtenu par simple transfert de charge entre donneurs et accepteurs localisés, dit nipi.

\subsubsection{Puits quantiques et super-réseaux : en quel sens?}

Les premières hétérostructures bidimensionnelles ont été réalisées couramment à la fin des années 70. Elles peuvent être qualifiées de bidimensionnelles au sens où les états électroniques sont confinés quantiquement. Il est nécessaire de prendre quelques précautions pour objectiver cette notion : il ne fait aucun doute que les états électroniques d'un semiconducteur massif ne peuvent s'expliquer que grâce à la mécanique quantique. Mais leurs 
propriétés sont en pratique indépendantes de la taille du cristal, car celle-ci est bien supérieure à la longueur de cohérence des états, limitée par des processus de diffusion inévitables, les imperfections du cristal, de sa surface, etc. Lorsque la largeur d'un "puits quantique" (Cf. fig.3.4 b, c) est nettement inférieure à cette longueur de cohérence, on peut parler d'effet de confinement quantique. En effet, la fonction enveloppe de l'état considéré subira aux limites du puits des conditions (par exemple d'annulation) qui se traduiront visiblement sur les observables. On voit donc que cette notion dépend des états considérés et aussi, secondairement, de la température et de la qualité de réalisation technologique. En pratique, les effets de confinement sont visibles sur les électrons de conduction dans GaAs, de masse effective 0.07 , en dessous de $30 \mathrm{~nm}$. Mais on peut aussi en parler pour des phonons optiques, en dessous de $3 \mathrm{~nm}$; ou bien pour des polaritons excitoniques qui sont des particules mixtes exciton-photon et dont la longueur de cohérence peut s'étendre comme celle des photons.

Dans le cas pédagogique du puits quantique de profondeur infinie et de largeur $\mathrm{L}$, les conditions aux limites (3.7) imposent l'annulation de la fonction d'onde à l'interface. Les états tridimensionnels de valence et de conduction se regroupent en sous-bandes d'états, de fonction enveloppe (Cf. éq. 3.5 et 3.8 ) :

$$
F_{\lambda, n, k_{\perp}}(\mathbf{r})=\mathrm{e}^{\mathrm{ik}} \perp, \mathbf{r}_{\perp} \varphi_{\lambda, \mathrm{n}}(\mathrm{z})
$$

et d'énergie :

$$
E_{n, k_{\perp}}=\hbar^{2} \pi^{2} n^{2} / 2 m_{e, h} L^{2}+\hbar^{2} k_{\perp}^{2} / 2 m_{e, h}
$$

Le mouvement libre dans le plan reste donc décrit par un vecteur d'onde bidimensionnel. La densité d'états de chaque sous-bande est évaluée de façon similaire au cas tridimensionnel (Cf. parag. 2.1): $\rho\left(\mathbf{k}_{\perp}\right) \mathrm{d}^{2} \mathbf{k}_{\perp}=(L / 2 \pi)^{2} \mathrm{~d}^{2} \mathbf{k}_{\perp}$, et donc, en supposant là aussi une loi de dispersion parabolique :

$$
\rho(E) d E=(L / 2 \pi)^{2} 2 m \pi / \hbar^{2} \sum_{n} \Theta\left(E-E_{n, 0}\right) d E
$$

où $\Theta(E)$ est la fonction de Heavyside. La densité d'état associée à chaque sous-bande bidimensionnelle est constante, et l'ensemble présente une discontinuité plus marquée qu'en 3D, au gap et au passage de chaque sous-bande.

À partir de puits quantiques répétés périodiquement (multipuits quantique), on parle de super-réseau lorsque des effets de superpériodicité ou de couplage entre puits sont attendus (Cf. fig.3.4 d, e).

Les états électroniques de super-réseau se décrivent dans le cadre de l'EFA de façon tout a fait analogue à une méthode des liaisons fortes appliquée uniquement dans la direction $\mathrm{z}$, analogue à ce qui est présenté au paragraphe 2.2.2. Contrairement au calcul de structures de bande des cristaux, cette approche simplissime donne d'ailleurs des résultats très satisfaisants. On fabrique des combinaisons linéaires de fonctions enveloppes de puits isolés qui ont la forme de Bloch :

$$
F_{\lambda, n, Q, k_{\perp}}(r)=\varphi_{\lambda, n}(z) e^{i Q z} e^{i k_{\perp} \cdot r_{\perp}}
$$

mais où $Q$ est maintenant un super-vecteur d'onde à une dimension. Il s'ensuit, à partir de chaque état lié du puits isolé, la formation d'une mini-bande de courbure positive ou négative en $\mathrm{Q}=0$ selon la parité de l'état de départ.

Là aussi, pour que cela ait une réalité physique, il faut que la sonde de cette périodicité reste cohérente sur plusieurs périodes. Cela pose moins de problèmes, par exemple, en optique ou en diffraction de rayons $X$, qu'avec les états électroniques. Comme on s'y attend, la tendance générale des effets de super-réseaux est d'atténuer le caractère bidimensionnel des 
états électroniques et des densités d'états, de tendre vers un système tridimensionnel anisotrope.

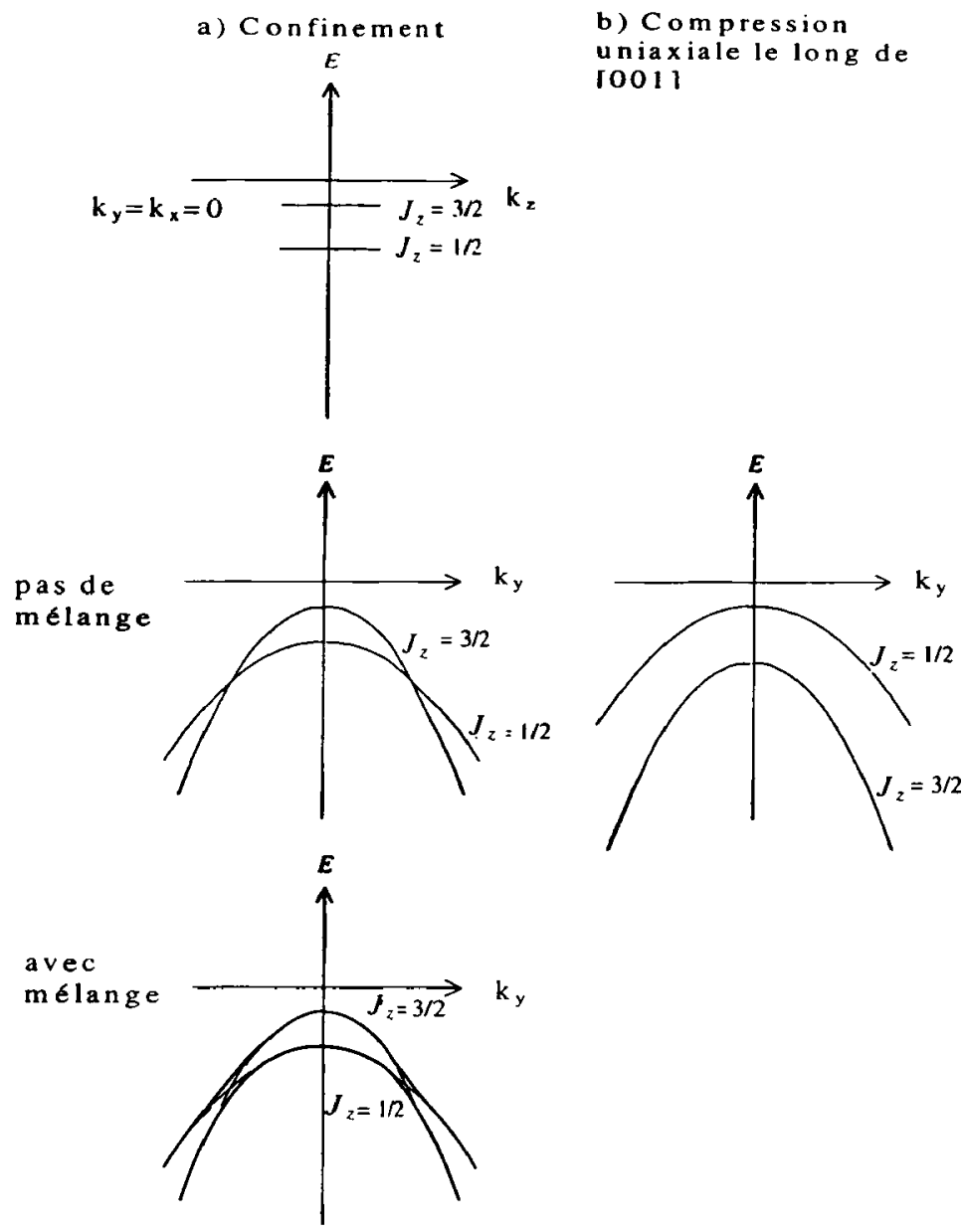

Figure 3.5 : Dispersion dans le plan des trous" lourds" et "légers" a) dans un puits quantique b) sous contrainte uniaxiale le long de l'axe [001], souvent la direction de croissance. Remarquez le croisement lourd/léger hors de $k=0$, qui devient anticroisement lorsqu'on inclut les termes de couplage. (Emprunté à Yu et Cardona [6] p.472)

\subsubsection{Diversité des systèmes bidimensionnels.}

Dans le système classique GaAs/GaAlAs, la proportion d'aluminium dans l'alliage est maintenue inférieure à environ $35 \%$ afin qu'il reste direct (Cf. fig. 3.1). Le profil de potentiel dessiné par les extrema de valence et de conduction, en $k=0$, est représenté sur la figure $(3.4 \mathrm{~b} \mathrm{~d}, \mathrm{e})$. On se trouve donc avec un puits de potentiel situé au même endroit dans la structure pour les électrons et pour les trous. C'est un profil de type I, dont on comprend déjà qu'il sera particulièrement favorable aux transitions optiques électron-trou. Pour dessiner un 
tel profil, il faut connaître les énergies des gaps, assez facilement accessibles expérimentalement ; mais il faut aussi connaître la position relative en énergie des structures de bandes des matériaux hôtes, ce qui n'est pas si facile : les expériences de mesure directe de ce décalage, comme la photoémission d'électrons, manquent beaucoup de précision, et les prévisions théoriques sont hasardeuses. En pratique, seule l'étude des propriétés électroniques et optiques d'hétérostructures astucieusement dessinées permettent d'accéder à ce décalage de bande avec une précision suffisante.

On exprime généralement cette position par un paramètre de décalage sans dimension qui est donné par la proportion du décalage de gap direct qui affecte la bande de conduction. L'intérêt d'utiliser une telle proportion vient du fait que, dans les "bons systèmes" dont les alliages suivent la loi de Végard, ce paramètre se révèle peu dépendant de la composition. Pour le système GaAs/GaAlAs, il est estimé entre 0.6 et 0.7 .

La situation est très différente, en particulier pour l'opticien, dans les structures dites de type II, où les états de base d'électrons et de trous ne sont pas confinés dans les mêmes couches (Cf. fig.3.4 f). D'une part les transitions optiques associées au gap fondamental seront moins fortes. D'autre part, à l'excitation lumineuse sera associé, après relaxation des porteurs, un transfert de charge, puisqu'électrons et trous ne seront pas situés dans les mêmes couches. Un système exemplaire, dans le domaine visible, est formé par le couple InP/AlInAs accordé, avec un gap assez proche pour les deux matériaux, et un décalage de bande d'environ $0,2 \mathrm{eV}$, qui donne des profils de potentiel "en chicane".

Un autre exemple de grande importance pratique est le système GaAs/AlAs lorsque sont concernés les états de bord de zone $X$ (et non les états de centre de zone $\Gamma$, qui forment un système de type I). Ce système présente aussi un intérêt heuristique pour la compréhension de l'Approximation de la Fonction Enveloppe et de ses limites (Cf. parag. 3.1.4). Le profil de potentiel des états $\Gamma$ de conduction donne naissance à une première série d'états confinés, dans les couches de GaAs. Un autre profil peut être tracé avec les états X, qui donne des états confinés dans les couches d'AlAs. Chacune de ces familles d'états peut être décrite dans l'esprit de l'EFA, en séparant les fonctions d'onde des matériaux massifs en une partie rapidement variable (attachée à l'extremum $\Gamma$ ou $\mathrm{X}$ ) et une partie lentement variable qui est modulée par le potentiel. On mesure là l'importance de la condition (3.2), qui nous dit qu'il faut marier les états $\Gamma$ de chaque matériau entre eux, et les états $X$ entre eux. En pratique, quand les couches de GaAs sont inférieures à $4 \mathrm{~nm}$, et les couches d'AlAs suffisamment épaisses, l'état fondamental de conduction est de type $\mathrm{X}$, situé dans l'AlAs. Mais décrire un quelconque couplage entre les deux familles va, par définition, au-delà de l'EFA. Expérimentalement, ce couplage perturbe le croisement des états $\Gamma$ et $X$ sur, typiquement, quelques meV; c'est dire l'étonnante validité de l'EFA.

Dans certains cas, le décalage de bande d'un système de type II est comparable aux gaps des matériaux, ce qui amène à une situation originale où le gap entre les énergies d'électrons dans l'un et les énergies de trous dans l'autre est très petit, voire nul. C'est le cas du système InAs/GaSb.

La distinction entre systèmes de type I et II devient plus floue, quand on prend en compte non plus seulement les profils de potentiel au sens strict, mais aussi les effets de confinements dans toutes leurs richesses, ce qui inclit la pénétration des fonctions d'ondes dans les barrières. Ainsi, on peut construire, avec un système de type I, des structures asymétriques, où électrons et trous ne sont pas localisés de la même façon, à cause de la différence de leur masse effective. En effet, dans beaucoup de cas, les trous lourds ont une masse de confinement (parallèle à la direction $z$ de croissance) supérieure d'un ordre de grandeur à celle 
des électrons. On comprend donc que les distributions de probabilités de présence de l'un et l'autre soient très différentes dans une structure notablement différente du puits carré infini. Ces quantités peuvent en conséquence faire l'objet d'un travail d'"ingénierie de structure de bande" sans équivalent dans les matériaux massifs, par exemple pour induire des nonlinéarités dues au transfert de charge. Ces structures asymétriques permettent d'ailleurs de jouer aussi avec les niveaux confinés successifs issus d'une même bande. On en verra des exemples intéressants pour l'optique intrabande au paragraphe 4.9

J'ai évoqué plus haut la possibilité de réaliser, dans certaines limites, la croissance pseudomorphique de matériaux contraints. La déformation associée n'est pas sans influence sur les énergies des bandes, en particulier en abaissant la symétrie du cristal. Ainsi, la dégénérescence trou lourd/trou léger est levée, hors tout effet de confinement, ce qui rajoute une tonalité supplémentaire à la palette de l'ingénieur (Cf. fig. 3.5). Un bon exemple en est GaInAs contraint sur GaAs, qui permet de repousser à plus haute énergie les trous légers et de bénéficier de la masse plus légère des trous lourds dans le plan, ce qui leur confère une meilleure mobilité. Une application se trouve dans les transistors à effet de champ (Cf. parag.1.2), Une autre application se trouve dans les lasers, pour adapter les densités d'états des électrons et des trous. Plus généralement, ce peut être un moyen de se débarrasser dans une certaine mesure de la complexité de la bande de valence. La description théorique de ces effets de contrainte est très bien maîtrisée mais un peu lourde. Je m'en dispenserai dans ce cours, renvoyant au cours de Marzin [22] qui donne les outils nécessaires.

Un autre type d'asymétrie est fourni par les structures piézoélectriques. Celles-ci sont obtenus par croissance de matériaux contraints sur des substrats orientés dans des directions qui s'y prêtent, par exemple [111]. La contrainte génère un champ électrique qui introduit donc un type d'asymétrie un peu comparable à celle du profil de potentiel de type II. Là aussi, l'illumination induit un transfert de charge, ou, si l'on préfere, un écrantage du champ électrique. Il en résulte une modification marquée du profil de potentiel et des effets non linéaires associés. Beaucoup de dispositifs ont été imaginés dans les systèmes GaInAs/GaAs et $\mathrm{CdZnTe} / \mathrm{CdTe}$.

Avec les semi-conducteurs magnétiques, (en pratique avec les semi-magnétiques), on peut réaliser des structures incluant des couches aimantées (paramagnétiques le plus souvent) localisée dans l'empilement. Grâce à l'interaction d'échange entre états électroniques localisés et états des bandes, il est donc possible de modifier sous champ magnétique le profil de potentiel effectif vu par les électrons (et les trous) d'un spin donné ; avec, bien entendu, une évolution opposée pour les états de spin conjugué.

Enfin, il ne faut pas oublier de prendre en compte les effets du dopage et du transfert de charge éventuellement associé dans les hétérostructures, par définition inhomogènes. Le dopage localisé, ou modulé, est d'ailleurs un outil supplémentaire pour l'ingénierie. Quelques exemples sont représentés sur la figure $3.4 \mathrm{a}, \mathrm{h}, \mathrm{i}$, dont la structure emblématique des expériences de transport quantique, l'hétérojonction à dopage modulé. Dans le domaine optique, il faut surtout citer les puits dopés pour l'optique intrabande (Cf. parag.4.9), et les structures $n-i-p-i$, qui, comme les structures asymétriques ou de type II, permettent d'envisager des non-linéarités associées au transfert de charge sous illumination. Dans tous ces cas, la modélisation quantitative des structures nécessitera la prise en compte du potentiel électrostatique relié à la densité de charge volumique par l'équation de Poisson. La densité de charge elle-même dépend de la probabilité de présence des états confinés, et la modification du potentiel de l'hétérostructure est en général suffisamment importante pour que le calcul des états confinés par l'équation de Schrödinger doive être repris (la méthode des matrices de 
transfert, présentée au paragraphe 3.3 est alors d'une grande utilité), et un traitement par itérations successives est le plus souvent inévitable.

\subsubsection{Fils et boîtes quantiques}

Dans les années 80 , on a vu se déployer de par le monde des trésors d'imagination, s'appuyant sur beaucoup de travail, pour réduire la dimensionnalité des objets réalisables. Il s'agissait donc de créer des fils et boîtes "quantiques", au sens explicité plus haut (Cf. parag. 3.2.1) pour le cas bidimensionnel. Aujourd'hui, le bilan est mitigé. Les méthodes les plus simples de lithographie ont prouvé leur efficacité dans de nombreux domaines, en particulier la physique du transport mésoscopique, mais les résultats pour l'optique sont un peu décevants. La raison principale en est sans doute les recombinaisons non radiatives induites par les flancs gravés. Les structures les plus intéressantes semblent obtenues lorsqu'on fait appel, peu ou prou, aux facultés d'auto-organisation de la croissance. Risquons nous à un bilan sans doute provisoire et certainement subjectif : les meilleurs fils quantiques sont aujourd'hui réalisés par croissance dans des sillons en V [23] ou par reprise d'épitaxie sur la tranche clivée d'un puits quantique [24], ce qu'on appelle les fils en T. Les ensembles d'objets obtenus ne sont pas très denses, ce qui pose problème pour de véritables utilisations en optique. Aujourd'hui, seules les croissances sur surface désorientée de quelques degrés [25], qui permettent d'accrocher des fils quantiques à chaque marche de la surface, peuvent fournir une densité très supérieure. Mais par contre, le confinement obtenu est assez faible.

Les meilleures boîtes quantiques semblent quant à elles obtenues par croissance épitaxiale en îlots [26] ou, noyées dans des matrices transparentes, par des techniques sol-gel (Cf. le cours de D.Ricard dans ce livre). On obtient des objets encore imparfaitement calibrés, mais en grande densité.

Il est facile de généraliser le calcul des énergies et densité d'états au cas 1 et 0 dimension, dans le modèle le plus simple de masse effective et de hauteur de barrière infinie. À $1 \mathrm{D}$, on en déduit que la densité d'état présente, à l'énergie de base de chaque sous-bande, une singularité en $\mathrm{E}^{-1 / 2}$, encore plus marquée qu'à $2 \mathrm{D}$. À $\mathrm{OD}$, on retrouve théoriquement une série discrète de niveaux d'énergie, comme pour un atome ou bien une impureté dans un semi-conducteur. Cependant, pour des barrières finies, les calculs sont beaucoup plus complexes qu'à $2 \mathrm{D}$ car l'équation de Schrödinger effective n'est plus séparable.

Cependant, bien que ces systèmes "quantiques" de dimension inférieure à 2 soient moins bien maîtrisés au plan de la technologie, beaucoup de travail a déjà été fait en optique non linéaire sur les nanocristaux noyés dans des matrices de verre, et leur qualité continue de progresser. C'en est donc le meilleur exemple à présenter aujourd'hui dans cette École.

\subsubsection{Structures optiques}

Les semi-conducteurs sont aussi des matériaux pour l'optique au sens où ils sont doués d'un indice de réfraction. Les techniques de dépôt de couches minces, très utilisées en optique, peuvent donc être employées dans certaines limites avec des semi-conducteurs, jouant avec les sauts d'indice pour définir des "structures optiques" tels que guide d'onde, miroir interférentiel ou résonateur Fabry-Perot. Dans les cas où le dépôt prend la forme d'une épitaxie, on peut y inclure des nanostructures électroniques (puits quantiques par exemple), mariant avec un bénéfice mutuel la physique des semi-conducteurs et l'optique. 
Cette démarche est présente depuis plusieurs années avec le laser à double hétérostructure et à puits quantiques, qui associe donc des puits de largeur nanométrique, de densité d'états électroniques bidimensionnelle, à un guide d'onde optique, de dimensions micrométriques. Le problème de l'accord de phase en optique non linéaire, abordé dans cette École, a inspiré récemment bon nombre de ces structures optiques. Une École y a été consacrée récemment, dont le volumineux compte rendu mérite une mention spéciale [27]. Ici, je présenterai en détail au paragraphe 4.7 les structures microcavités où excitons et photons sont couplés fortement.

\subsection{Calcul des états électroniques des hétérostructures bidimensionnelles}

Je voudrais dans ce paragraphe exposer un ensemble cohérent de méthodes d'intérêt pratique pour calculer les états confinés dans une hétérostructure bidimensionnelle. Par "méthodes d'intérêt pratique", j'entends suffisamment élaborées pour souffrir la comparaison avec l'expérience. Si l'on considère en effet les systèmes les mieux connus, comme le système $\mathrm{GaAs} / \mathrm{GaAlAs}$, avec une bonne maîtrise de la technologie et une connaissance raisonnable des paramètres de base des matériaux hôtes, l'intérêt d'aller au-delà de la méthode de calcul de Ben Daniel-Duke (masse effective constante, mais différente d'un matériau à l'autre) ne fait aucun doute. Cependant, toutes ces méthodes se situent dans le cadre général de l'Approximation de la Fonction Enveloppe (EFA), qui a largement prouvé son utilité dans tous les cas où les états électroniques sont raisonnablement délocalisés. Naturellement, aller trop loin dans la sophistication pourrait relever de l'exercice gratuit; il faut savoir faire preuve de sens physique.

\subsubsection{Traitement d'un profil de potentiel quelconque. Matrice de transfert}

Il est vite indispensable de pouvoir traiter un profil de potentiel plus compliqué que celui d'un puits carré ou d'un super-réseau en créneau. D'une part pour les besoins de «l'ingénierie de bande ", qui peut amener à construire des structures de formes plus riches, et/ou à appliquer un champ électrique le long de l'axe de croissance. D'autre part pour prendre en compte certaines imperfections technologiques inévitables, comme la gradualité des interfaces, par exemple dans le système GaInAs/GaAs. On peut employer selon les cas des méthodes perturbatives ou variationnelles, souvent riches de sens physique. Mais la méthode la plus utile pour les structures bidimensionnelles, en particulier par la généralité de son traitement numérique, est celle des matrices de transfert [28].

La méthode consiste à découper le profil en sections de potentiel constant, aussi fines qu'il est nécessaire dans le cas d'une gradualité ou d'un champ électrique. On l'aura compris, il ne s'agit pas ici de se préoccuper outre mesure ni de la discrétisation naturelle en mailles élémentaires, ni de la condition de validité de l'EFA, qui stipule que le potentiel doit varier lentement à cette échelle. On l'a déjà dit (cf. parag. 3.1.4), il apparaît que cette condition est suffisante, mais pas nécessaire lorsque la fonction enveloppe finalement calculée varie, elle, lentement. Chaque tranche ainsi définie sera caractérisée donc par une énergie potentielle $V_{i}$ et une masse effective $\mathrm{m}_{\mathrm{i}}(\varepsilon)$, qui peut dépendre de l'énergie. Nous nous autorisons à considérer au départ des états de $k$ non nul, avec une masse dans le plan $m_{\perp, i}(\varepsilon)$ non nécessairement identique à la masse de confinement $m_{i}$. Pour trouver les états stationnaires de 
l'énergie, la structure sera toujours entourée de barrières suffisamment hautes et épaisses, qui représenteront soit des couches de confinement ad hoc, soit le vide, et qui assureront l'annulation de la fonction enveloppe à l'infini. (Mais d'autres conditions aux limites peuvent être mieux adaptées à la situation physique : conditions périodiques BVK pour des superréseaux infinis, ondes planes à l'infini pour des expériences de transmission.)

La méthode consiste à fixer une énergie $\varepsilon$ et à suivre de proche en proche l'évolution de l'onde électronique d'énergie $\mathrm{E}$ à partir de la première barrière, puis au travers de toutes les tranches de la structure. Les états propres seront ceux qui satisfont aux conditions aux limites dans l'ensemble de la structure, généralement d'être bornée à l'infini.

L'évolution de la fonction enveloppe dans chaque tranche $\mathrm{i}$ est régie par l'équation de Schrödinger : par contre, nous supposerons que les mouvements // et $\perp$ sont séparables, d'éventuels termes de couplage étant par la suite traités en perturbation, comme on le verra dans le cas de la bande de valence :

$$
\left[-\frac{\hbar^{2}}{2 m_{i}} \frac{\partial^{2}}{\partial z^{2}}+\frac{\hbar^{2} k_{\perp}^{2}}{2 m_{\perp, i}}+V_{i}\right] \varphi_{i}(z)=\varepsilon \varphi_{i}(z) \text { dans la tranche i où } z_{i}<z<z_{i+1}
$$

La fonction enveloppe peut s'écrire, sous sa forme la plus générale et symétrique :

$$
\varphi_{i}(z)=a_{i} e^{\left[i k_{i}\left(z-z_{i}-L_{i} / 2\right)\right]}+b_{i} e^{\left[-i k_{i}\left(z-z_{i}-L_{i} / 2\right)\right]}
$$

où $L_{i}=z_{i+1}-z_{i}$. Notons surtout que l'énergie cinétique en $z: \frac{\hbar^{2} k_{i}^{2}}{2 m_{i}(\varepsilon)}=\varepsilon-\frac{\hbar^{2} k_{\perp}^{2}}{2 m_{\perp, i}(\varepsilon)}-V_{i}$ peut être positive ou négative, selon que l'onde est propagative (onde plane) ou évanescente (exponentielle décroissante).

Pour une énergie $\mathrm{E}$ donnée, la propagation de l'onde est donc parfaitement déterminée dans la couche i par cette équation de Schrödinger, et à l'interface, par les conditions aux limites (éq. 3.7) :

$$
\begin{gathered}
\varphi_{\mathrm{i}}\left(\mathrm{z}_{\mathrm{i}+1}\right)=\varphi_{\mathrm{i}+1}\left(\mathrm{z}_{\mathrm{i}+1}\right) \\
\mathrm{m}_{\mathrm{i}}^{-1}(\varepsilon) \varphi_{\mathrm{i}}^{\prime}\left(\mathrm{z}_{\mathrm{i}+1}\right)=\mathrm{m}_{\mathrm{i}+1}^{-1}(\varepsilon) \varphi_{\mathrm{i}+1}^{\prime}\left(\mathrm{z}_{\mathrm{i}+1}\right)
\end{gathered}
$$

L'ensemble formé par les équations (3.12) et (3.13) permet d'obtenir $a_{i}$ et $b_{i}$ en fonctions de $a_{i-1}$ et $b_{i-1}$ par un système linéaire, représentée par une "matrice de transfert" $\mathbf{M}_{\mathbf{i}}$ ne dépendant que des paramètres des couches $\mathbf{i}$ et $\mathbf{i}+1$. La fonction enveloppe est donc connue de proche en proche par:

$$
\left[\begin{array}{l}
a_{i} \\
b_{i}
\end{array}\right]=\prod_{j=i-1}^{j=0} \mathbf{M}_{j}\left[\begin{array}{l}
a_{0} \\
b_{0}
\end{array}\right]
$$

Cependant, comme toujours dans un système confiné, toute énergie $E$ ne permet pas de construire une fonction propre ; ce sont les conditions aux limites qui en décideront. Ici, on veut que la fonction soit évanescente dans les deux barrières extrêmes, indexées 0 et $\mathrm{N}$. 
On en déduit que $\left[\begin{array}{l}a_{0} \\ b_{0}\end{array}\right]=\left[\begin{array}{l}0 \\ 1\end{array}\right]$ et $\left[\begin{array}{l}a_{N} \\ b_{N}\end{array}\right]=\left[\begin{array}{l}1 \\ 0\end{array}\right]$ (à une normalisation près). Donc, la matrice de transfert totale $\mathbf{M}=\prod_{j=N}^{\mathrm{j}=0} \mathbf{M}_{\mathbf{j}}$ doit satisfaire : $\left[\begin{array}{l}1 \\ 0\end{array}\right]=\mathbf{M}\left[\begin{array}{l}0 \\ 1\end{array}\right]$, ce qui impose que son élément $\mathbf{M}_{22}=0$.

Telle est donc l'équation séculaire qui autorise les valeurs propres de l'énergie. Ensuite, le calcul (et la normalisation) de la fonction d'onde ne pose pas de problèmes autres que numériques, ce qui peut tout de même nécessiter quelque attention pour les structures complexes.

\subsubsection{Non parabolicité des bandes. Méthode de Kane}

Tableau 3.1 : Hamiltonien de Kane à 4 bandes pour les semi-conducteurs de type GaAs, ZnTe.... L'origine des énergies est située en haut de la bande de valence. (Emprunté à G.Bastard, [13]).

\begin{tabular}{|c|c|c|c|c|c|c|c|c|}
\hline & $\mid$ is $\uparrow\rangle$ & $|3 / 2,1 / 2\rangle$ & $|3 / 2,3 / 2\rangle$ & $|1 / 2,1 / 2\rangle$ & $\mid$ is $\downarrow\rangle$ & $|3 / 2,-1 / 2\rangle$ & $|3 / 2,-3 / 2\rangle$ & || $1 / 2,-1 / 2\rangle$ \\
\hline is $\uparrow\rangle$ & $\begin{array}{l}E_{G} \\
+\frac{\hbar^{2} k^{2}}{2 m_{0}}\end{array}$ & $-\sqrt{\frac{2}{3}} \frac{\mathrm{P} \hbar \mathrm{k}_{\mathrm{z}}}{\mathrm{m}_{0}}$ & $\frac{\mathrm{P} \hbar \mathrm{k}_{+}}{\mathrm{m}_{0}}$ & $\sqrt{\frac{1}{3}} \frac{\mathrm{P} \hbar \mathbf{k}_{\mathbf{z}}}{\mathrm{m}_{0}}$ & 0 & $-\sqrt{\frac{1}{3}} \frac{\mathrm{P} h \mathrm{k}_{-}}{\mathrm{m}_{0}}$ & & $-\sqrt{\frac{2}{3}} \frac{\mathrm{P} \hbar \mathrm{k}-}{\mathrm{m}_{0}}$ \\
\hline$|3 / 2,1 / 2\rangle$ & $-\sqrt{\frac{2}{3}} \frac{\mathrm{P} \hbar \mathrm{k}_{\mathrm{z}}}{\mathrm{m}_{0}}$ & $\frac{\hbar^{2} k^{2}}{2 m_{0}}$ & 0 & 0 & $\sqrt{\sqrt{\frac{1}{3}}} \frac{\mathrm{P} h \mathrm{k}_{-}}{\mathrm{m}_{0}}$ & 0 & 0 & 0 \\
\hline$|3 / 2,3 / 2\rangle$ & $\frac{\mathrm{P} \hbar \mathrm{k}_{-}}{\mathrm{m}_{0}}$ & 0 & $\frac{\hbar^{2} \mathrm{k}^{2}}{2 \mathrm{~m}_{0}}$ & 0 & 0 & 0 & 0 & 0 \\
\hline $1 / 2,1 / 2\rangle$ & $\sqrt{\frac{1}{3}} \frac{P \hbar \mathbf{k}_{\mathbf{z}}}{\mathrm{m}_{0}}$ & 0 & 0 & $\begin{array}{l}-\Delta s o \\
+\frac{\hbar^{2} k^{2}}{2 m_{0}} \\
\end{array}$ & $\sqrt{\frac{2}{3}} \frac{P \text { hk }-}{m_{0}}$ & 0 & 0 & 0 \\
\hline is $\downarrow\rangle$ & 0 & $\sqrt{\frac{1}{3}} \frac{\mathrm{P} \hbar \mathrm{k}_{-}}{\mathrm{m}_{0}}$ & 0 & $\sqrt{\frac{2}{3}} \frac{\mathrm{P} \hbar \mathrm{k}+}{\mathrm{m}_{0}}$ & $\begin{array}{l}\mathrm{E}_{G_{G}} \\
+\frac{\hbar^{2} k^{2}}{2 m_{0}}\end{array}$ & $-\sqrt{\frac{2}{3}} \frac{\mathrm{Phk_{z }}}{\mathrm{m}_{0}}$ & $\frac{\mathrm{P} h \mathrm{k}_{-}}{\mathrm{m}_{0}}$ & $\sqrt{\frac{1}{3}} \frac{P \hbar \mathrm{k}_{\mathrm{z}}}{\mathrm{m}_{0}}$ \\
\hline$|3 / 2,-1 / 2\rangle$ & $-\sqrt{\frac{1}{3}} \frac{P \hbar k_{+}}{m_{0}}$ & 0 & 0 & 0 & $-\sqrt{\frac{2}{3}} \frac{\mathbf{P} \hbar \mathbf{k}_{\mathrm{z}}}{\mathrm{m}_{0}}$ & $\frac{\hbar^{2} \mathrm{k}^{2}}{2 \mathrm{~m}_{0}}$ & 0 & 0 \\
\hline$|3 / 2,-3 / 2\rangle$ & 0 & 0 & 0 & 0 & $\frac{\mathrm{P} \hbar \mathrm{k}_{+}}{\mathrm{m}_{0}}$ & 0 & $\frac{\hbar^{2} \mathrm{k}^{2}}{2 \mathrm{~m}_{0}}$ & 0 \\
\hline$|1 / 2,-1 / 2\rangle$ & $-\sqrt{\frac{2}{3}} \frac{\mathrm{P} \hbar \mathrm{k}_{+}}{\mathrm{m}_{0}}$ & 0 & 0 & 0 & $\sqrt{\frac{1}{3}} \frac{P \hbar k_{z}}{m_{0}}$ & 0 & 0 & $\begin{array}{l}-\Delta \text { so } \\
+\frac{\hbar^{2} \mathrm{k}^{2}}{2 \mathrm{~m}_{0}}\end{array}$ \\
\hline
\end{tabular}

La méthode de perturbation de Kane [29, YC.p.64] permet d'obtenir une description des états de bande assez satisfaisante autour d'un extremum, le terme de perturbation étant l'opérateur k.p. Elle a été d'abord utilisée pour obtenir les masses effectives des semi-conducteurs 
massifs. Mais, étant valable aux petits $k$ et foumissant des résultats analytiques, elle est particulièrement adaptée à l'esprit de l'EFA, dont elle forme d'ailleurs l'une des fondations théoriques possibles [13]. Ici, nous nous contenterons d'en tirer des valeurs de masse effective dépendant de l'énergie, qu'il sera possible d'"injecter" dans un calcul EFA, utilisant par exemple les matrices de transfert.

Une fois de plus, je prendrai exemple sur la structure de bande la plus courante des matériaux composés de type zinc-blende, avec comme point de départ l'ensemble bien connu des $4 \times 2$ bandes de conduction et de valence (Cf. parag. 2.2.1 et fig. 2.2) qui diagonalise l'interaction spin-orbite. La méthode consiste à considérer cet ensemble comme suffisamment éloigné des autres bandes pour constituer un sous-espace pertinent. On peut alors y diagonaliser le hamiltonien perturbé. Plus précisément, les fonctions d'onde (de Bloch) sont de la forme :

$$
\varphi_{\lambda, k}(\mathbf{r})=\mathbf{u}_{\lambda, k}(\mathbf{r}) \mathrm{e}^{\mathrm{ik} . \mathbf{r}}, \text { fonction propre du hamiltonien total } \mathbf{H}_{\text {total }}=\left[\frac{1}{2 \mathrm{~m}_{0}} \mathbf{p}^{2}+\mathrm{V}\right]
$$

mais dont la partie périodique $u_{\lambda, k}(\mathbf{r})$ est fonction propre du hamiltonien de Kane :

$$
\mathbf{H}_{\text {Kane }} \mathrm{u}_{\lambda, \mathbf{k}}(\mathbf{r})=\left[\frac{1}{2 \mathrm{~m}_{0}}\left(\mathbf{p}^{2}+2 \hbar \mathbf{k} \cdot \mathbf{p}+\hbar^{2} \mathbf{k}^{2}\right)+\mathrm{V}\right]=\mathrm{E}_{\lambda, \mathbf{k}} \mathrm{u}_{\lambda, \mathbf{k}}(\mathbf{r})
$$

Il est présenté sous forme matricielle dans le tableau 3.1, sur la base des fonctions $u_{n 0}$, supposées connues. L'origine des énergies est prise en haut de la $\mathrm{BV}, \mathrm{E}_{\mathrm{G}}$ est le gap et $\Delta_{\mathrm{So}}$ l'interaction spin-orbite. Naturellement, on a quantifié l'opérateur $\mathrm{J}$ le long de $k$, ce qui peut toujours se faire dans le semi-conducteur massif. Les éléments de matrice des opérateurs $p$ seront à nouveau rencontrés et discutés à propos des règles de sélection des transitions optiques interbandes (parag. 4.2.1). P est le fameux élément de matrice de Kane :

$$
\mathrm{iP}=\langle\mathrm{S}|\mathrm{p}| \mathrm{X}\rangle=\langle\mathrm{S}|\mathrm{p}| \mathrm{Y}\rangle=\langle\mathrm{S}|\mathrm{p}| \mathrm{Z}\rangle
$$

En pratique, dans la plupart des matériaux usuels, $\mathrm{P}^{2} / \mathrm{m}_{0} \approx 10 \mathrm{eV}$. Ce fait est discuté de façon intéressante dans [YC. p.66].

Les quatre valeurs propres de l'énergie (dégénérées deux fois par renversement du temps) s'obtiennent en annulant le déterminant. En posant par commodité $\mathrm{E}^{*}(\mathbf{k})=\mathrm{E}(\mathbf{k})-\hbar^{2} \mathrm{k}^{2} / 2 \mathrm{~m}_{0}$, il vient :

- d'une part :

$$
\mathrm{E}^{*}=0,
$$

c'est la bande de trous lourds HH, non affectée par la perturbation, car sa fonction d'onde "atomique" pointe perpendiculairement à $\mathrm{k}$.

- d'autre part :

$$
\left(\mathrm{E}^{*}-\mathrm{E}_{\mathrm{G}}\right) \mathrm{E}^{*}\left(\mathrm{E}^{*}+\Delta_{\mathrm{SO}}\right)=\hbar^{2} \mathrm{k}^{2} \mathrm{P}^{2} / \mathrm{m}_{0}^{2}\left(\mathrm{E}^{*}+2 \Delta_{\mathrm{SO}} / 3\right)
$$

dont les trois racines sont les trois bandes légères $\mathrm{BC}, \mathrm{LH}$ et SO. On obtient explicitement $k(E)$ sous forme analytique, ce qui est la quantité qu'il nous faut dans un calcul de matrice de transfert, par exemple. La tendance générale est à un alourdissement de la masse avec l'énergie cinétique, d'autant plus marqué que le gap est petit. Notons qu'il est possible aussi d'imposer l'énergie dans le gap $\left(E^{*}-E_{G}\right)<0$, ce qui fournit la relation entre l'énergie et le vecteur d'onde imaginaire des ondes évanescentes dans le matériau considéré et de prendre en compte aussi ces effets de non-parabolicité dans l'EFA. 
Cette description des bandes légères est raisonnable tant que les énergies cinétiques restent faibles par rapport à l'espacement des autres bandes non incluses dans cette théorie "à quatre bandes". Malheureusement, on ne décrit pas la bande de trous lourds ; il faudrait pour cela inclure le couplage avec les autres bandes plus éloignées, ce qui est beaucoup plus pénible. C'est pourquoi l'approche de Kane sera en pratique utilisée pour calculer les niveaux confinés de conduction, et aussi les niveaux de valence "légers" de $k_{\perp}=0$. Mais pour prendre en compte la complexité des bandes de valence, par exemple pour décrire les états de $k_{\perp} \# 0$, il faut faire appel au hamiltonien phénoménologique de Luttinger.

\subsubsection{Dégénérescence de la bande de valence. Hamiltonien de Luttinger}

Il s'agit donc maintenant de décrire analytiquement, mais phénoménologiquement, le haut de la bande de valence, en pratique les deux bandes $\mathrm{J}=3 / 2$ dans l'approximation parabolique ; nous disposons pour cela du hamiltonien introduit par Luttinger [30,YC p.164], le plus général qui soit construit avec les vecteurs $\mathbf{k}$ et $\mathbf{J}$, quadratique en $\mathbf{k}$ et invariant par les opérations de symétrie du cube (en toute rigueur, il faut préciser que l'absence de centre d'inversion de la structure zinc-blende est négligée) :

$$
\mathbf{H}_{\text {Luttinger }}=\frac{\hbar^{2}}{2 \mathrm{~m}_{0}}\left[\left(\gamma_{1}+5 / 2 \gamma_{2}\right) \mathbf{k}^{2}-2 \gamma_{2}(\mathbf{k} \cdot \mathbf{J})^{2}+2\left(\gamma_{3}-\gamma_{2}\right)\left(\mathbf{k}_{x}^{2} \mathrm{~J}_{x}^{2}+\text { perm.circ. }\right)\right]
$$

dont les coefficients sont exprimés en fonction des paramètres de Luttinger. C'est ainsi qu'on décrit aussi la non-sphéricité de la bande de valence (warping).

Voici les valeurs de ces paramètres pour quelques matériaux d'intérêt courant [31] :

$\begin{array}{llll} & \boldsymbol{\gamma}_{\mathbf{1}} & \boldsymbol{\gamma}_{\mathbf{2}} & \boldsymbol{\gamma}_{3} \\ \text { GaAs } & 7.65 & \mathbf{2 . 4 1} & 3.28 \\ \text { AlAs } & 3.45 & \mathbf{0 . 6 8} & 1.29 \\ \text { CdTe } & 5.29 & \mathbf{1 . 8 9} & 2.46 \\ \text { ZnTe } & 3.77 & \mathbf{1 . 0 7} & 1.64 \\ \text { ZnS } & 2.54 & \mathbf{0 . 7 5} & 1.09\end{array}$

L'approximation sphérique, souvent suffisante, consiste à écrire $\gamma_{2}=\gamma_{3}=\left(3 \gamma_{3}+2 \gamma_{2}\right) / 5$

Ce hamiltonien s'écrit facilement sous forme matricielle, en explicitant les éléments de matrice des opérateurs de type $\mathbf{J}^{2}$ sur la base des états dont le moment de rotation est quantifié le long de $z: J=3 / 2, m=3 / 2,1 / 2,-1 / 2,-3 / 2$. Je rappelle que cette base s'impose naturellement dans l'hétérostructure $2 \mathrm{D}$, puisque le confinement va lever la dégénérescence entre $\pm 3 / 2$ (trous lourds) et $\pm 1 / 2$ (trous légers). 


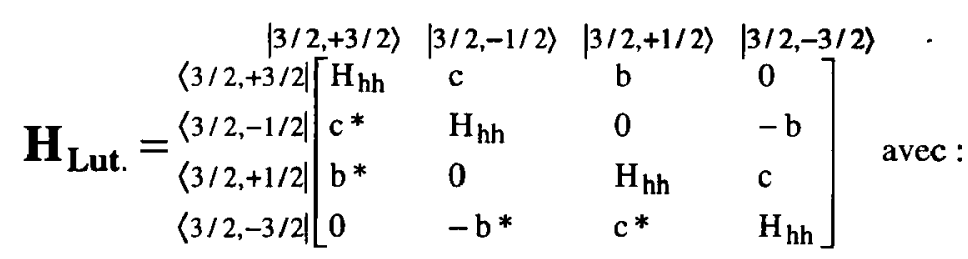

$$
\begin{aligned}
& \mathbf{H}_{\mathrm{hh}}=\frac{-\hbar^{2}}{2 \mathrm{~m}_{0}}\left[\left(\gamma_{1}-2 \gamma_{2}\right) \mathrm{k}_{\mathrm{z}}^{2}+\left(\gamma_{1}+\gamma_{2}\right) \mathrm{k}_{\perp}^{2}\right] \\
& \mathbf{H}_{\mathrm{lh}}=\frac{-\hbar^{2}}{2 \mathrm{~m}_{0}}\left[\left(\gamma_{1}+2 \gamma_{2}\right) \mathbf{k}_{\mathrm{z}}^{2}+\left(\gamma_{1}-\gamma_{2}\right) \mathbf{k}_{1}^{2}\right] \\
& c\left(k_{\perp}\right)=\frac{\hbar^{2} \sqrt{3}}{2 m_{0}}\left[\gamma_{2}\left(k_{x}^{2}-k_{y}^{2}\right)-2 i \gamma_{3} k_{x} k_{y}\right] \\
& \mathrm{b}\left(\mathbf{k}_{\perp}, \mathbf{k}_{\mathrm{z}}\right)=\frac{\hbar^{2} \sqrt{3}}{2 \mathrm{~m}_{0}}\left(\mathbf{k}_{\mathrm{x}}-\mathrm{ik} \mathrm{y}\right)\left(2 \gamma_{3} \mathrm{k}_{\mathrm{z}}\right)
\end{aligned}
$$

où $\mathrm{m}_{0}$ est, rappelons le, la masse de l'électron libre.

Nous en tirons d'abord les masses effectives pour les mouvements parallèle et perpendiculaire à l'axe de croissance $z$ :

$$
\begin{aligned}
& \mathrm{m}_{\mathrm{hh}, / /}=\mathrm{m}_{0} /\left(\gamma_{1}-2 \gamma_{2}\right) \\
& \mathrm{m}_{\mathrm{lh}, /}=\mathrm{m}_{0} /\left(\gamma_{1}+2 \gamma_{2}\right) \\
& \mathrm{m}_{\mathrm{hh}, \perp}=\mathrm{m}_{0} /\left(\gamma_{1}+\gamma_{2}\right) \\
& \mathrm{m}_{\mathrm{hh}, \perp}=\mathrm{m}_{0} /\left(\gamma_{1}-\gamma_{2}\right)
\end{aligned}
$$

Dans GaAs par exemple, ces masses ont pour valeur, en unité de $\mathrm{m}_{0}$ :

$$
\mathrm{m}_{\mathrm{hh}, / /} \sim 0.35, \mathrm{~m}_{\mathrm{lh}, / /} \sim 0.08, \mathrm{~m}_{\mathrm{hh}, \perp} \sim 0.1, \mathrm{~m}_{\mathrm{lh}, \perp} \sim 0.19
$$

On voit donc que les trous lourds sont relativement légers dans le plan des couches et réciproquement (dans les milieux de l'optique, il me semble que les termes "trous lourds et légers" sont malgré tout utilisés largement, et toujours par référence à la masse de confinement).

Cette situation de "renversement de masse" à des conséquences visibles sur la figure 3.5 . Dans les puits quantiques standard, où les trous lourds ont une moindre énergie de confinement, il y a croisement des deux bandes en $\mathbf{k}_{\perp} \neq 0$.

Pour faire usage du hamiltonien de Luttinger ci-dessus dans un calcul de fonction enveloppe, il faut bien entendu ajouter un potentiel $V$ variable en $z$, et remplacer les termes en $\mathrm{k}_{\mathrm{z}}$ par l'opérateur dérivation. En pratique, comme dans le modèle de Ben Daniel-Duke, on va se permettre d'utiliser des coefficients $\gamma$ de Luttinger variables en $z$. Il faut donc symétriser l'opérateur pour conserver son hermiticité. Donc, dans les expressions (3.17), on remplacera : - dans les termes diagonaux :

$$
\frac{\hbar^{2} \mathrm{k}_{\mathrm{z}}^{2}}{2 \mathrm{~m}_{0}} \gamma \text { par } \frac{1}{2 \mathrm{~m}_{0}} \mathrm{p}_{\mathrm{z}} \gamma(\mathrm{z}) \mathrm{p}_{\mathrm{z}}+\mathrm{V}(\mathrm{z})
$$

- et dans le terme $b$ :

$$
\hbar \mathrm{k}_{\mathrm{z}} \gamma \operatorname{par} \frac{1}{2}\left(\mathrm{p}_{\mathrm{z}} \gamma(\mathrm{z})+\gamma(\mathrm{z}) \mathrm{p}_{\mathrm{z}}\right)
$$


Ensuite, on pourra construire, par exemple par la méthode des matrices de transfert, les fonctions propres des équations de Schrödinger correspondant aux termes diagonaux :

$\mathrm{H}_{\mathrm{hh}} \varphi=\mathrm{E} \varphi$ et $\mathrm{H}_{\mathrm{lh}}=\mathrm{E} \varphi$, dont les solutions sont les fonctions enveloppes des états de trous lourds et légers non couplés, et les énergies propres sont les termes diagonaux de la matrice (3.17). On peut maintenant, dans un esprit de perturbation, en calculer les termes nondiagonaux (seul b dépend explicitement de la fonction enveloppe en $z$ ), et la diagonaliser pour trouver les nouveaux états couplés.
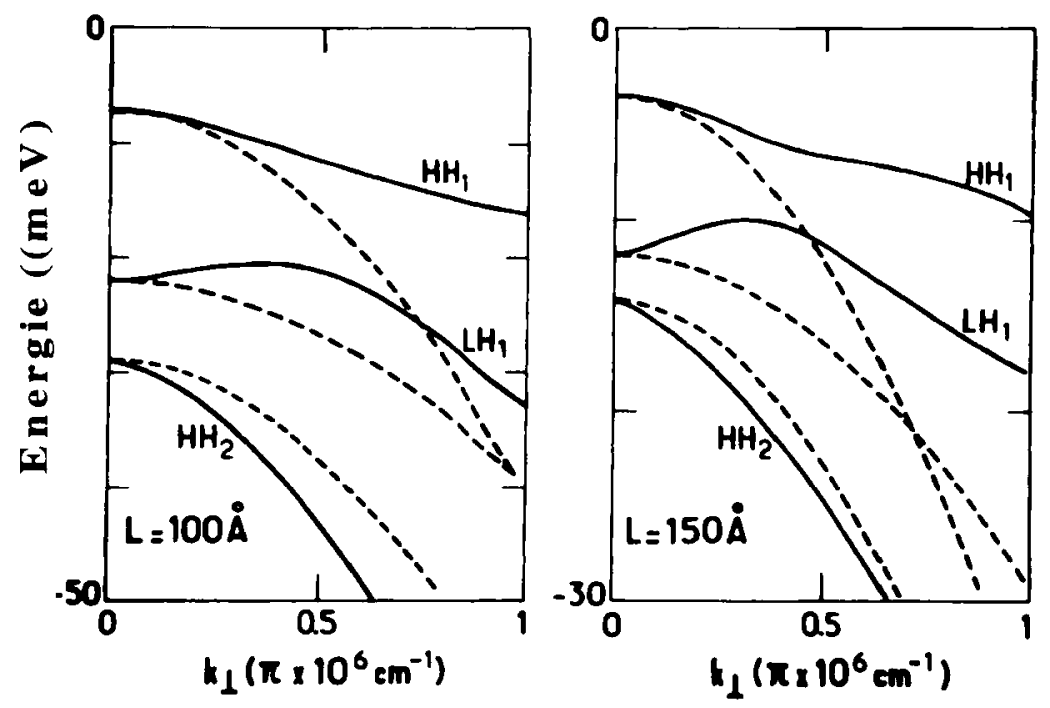

Figure 3.6 : Détail de la dispersion dans le plan des trous lourds et légers, calculés dans deux puits quantiques différents ( GaAs/GaAlAs) par G.Bastard [13]. Avec les courbes en pointillés, on néglige les termes non diagonaux du Hamiltonien de Luttinger, qui expriment le couplage trous lourds-légers.

À titre d'illustration, considérons les résultats d'un tel couplage sur la figure 3.6. Le fait le plus marquant est le dos de chameau des trous légers, qui se traduit par un pic de densité d'état bien commode pour la caractérisation optique. Ce calcul est réalisé dans des puits rectangulaires, symétriques. Il faut noter que, dans une structure non symétrique, lorsque le terme $b$ n'est pas nul, les dégénérescences entre $\pm 3 / 2$ d'une part, $\pm 1 / 2$ d'autre part (dégénérescences de spin) sont levées hors de $\mathbf{k}_{\perp}=0$. Ce résultat est discuté en détail par Bastard [13] et Altarelli [14].

\section{PROPRIÉTÉS OPTIQUES}

Dans l'approche semi-classique des propriétés optiques, la plus courante, les états électroniques sont décrits en mécanique quantique mais le champ électromagnétique (de fréquence $\omega$ et vecteur d'onde $\mathbf{q}$ ) est traité classiquement. La forme du hamiltonien 
d'interaction, en jauge de Coulomb, la plus utilisée dans les solides est : $H_{e r}=\frac{\mathbf{e}}{\mathbf{m}} \mathbf{A} \cdot \mathbf{p}$ où $\mathbf{A}$ est le potentiel vecteur du champ oscillant $\mathbf{E}$ :

$$
\mathbf{A}=-\frac{\mathbf{E}}{2 \mathrm{q}}[\mathrm{e} i(\mathbf{q} \mathbf{r}-\omega t)+\text { c.c. }]
$$

et l'interaction est traitée en perturbation dépendant du temps.

Ce hamiltonien est évidemment commode dans un système physique comportant des états délocalisés. On peut montrer [32] qu'il se réduit, dans le cadre de l'approximation dipolaire où le vecteur d'onde de la lumière est négligé, à $\mathrm{H}_{\mathrm{er}}=-\mathrm{er} . \mathrm{E}$, dont la signification physique est plus parlante, du moins pour un dipôle ponctuel.

Selon le régime temporel étudié, régime cohérent (effet Stark optique par exemple) ou au contraire obtention d'un état excité en quasi-équilibre (création de paires électron-trou), la partie dépendant du temps de ce hamiltonien fera l'objet d'un traitement différent. Cette discussion est introduite dans cette École par J.Y.Bigot. Je vais dans la suite discuter l'intensité du couplage et emprunterai des exemples à des expériences quasi continues d'absorption et de luminescence, donc relatives au régime des durées longues. Et ceci dans l'esprit de la remarque faite en fin de section 1.

\subsection{Eléments de matrice de l'opérateur $p$}

L'intensité du couplage, donc les propriétés optiques, vont reposer sur les éléments de matrice du produit scalaire A.p , qui connectent un état initial $\psi_{\mathrm{i}}$ et un état final $\psi_{\mathrm{f}}$ de la transition : $\left\langle\psi_{\mathrm{f}}|\mathbf{A} \cdot \mathbf{p}| \psi_{\mathrm{i}}\right\rangle$. En développant ce produit scalaire, il vient :

$$
H_{e r} \propto A \cdot p=A_{x} p_{x}+A_{y} p_{y}+A_{z} p_{z}=A_{+} p_{+}+A_{-} p_{-}+A_{z} p_{z}
$$

ce qui permet de décrire la relation entre la polarisation du champ et les états couplés. La première écriture est bien adaptée à une excitation polarisée linéairement, et la seconde, construite à partir de combinaisons linéaires ad hoc, à une excitation polarisée circulairement dans le plan $x, y$.

Comme on l'a vu, les états du semi-conducteur massif idéal prennent la forme de Bloch (Cf. éq. 2.1) ; de plus, les états d'impuretés peu profondes comme ceux des hétérostructures sont construits sur cette base dans le cadre de l'approximation de la fonction enveloppe. On va donc s'intéresser aux fonctions d'onde de la forme $\left|\psi_{i} / f\right\rangle=F_{1 / f} u_{i / f}$, où $u$ est une fonction rapidement variable, périodique comme le réseau cristallin (Cf. parag. 3.1.4); comme d'habitude, nous supposerons que nous traitons des états proches d'un extremum de la bande et négligerons sa dépendance en $\mathrm{k}$ autour de cet extremum. F est la fonction enveloppe, solution d'une équation de Schrödinger effective de type (3.4).

Pour calculer l'élément de matrice $\left\langle\psi_{\mathrm{f}}|\mathbf{A} \cdot \mathbf{p}| \psi_{\mathrm{i}}\right\rangle$, on peut procéder rigoureusement dans l'esprit de la théorie quantique des solides, en remplaçant l'intégrale sur tout l'espace par une somme d'intégrales (égales) sur la cellule unité, pondérées par la valeur de la fonction enveloppe sur le site (YC p.248). On peut aussi aller plus vite à la discussion physique en supposant, une fois de plus, que la fonction enveloppe et le champ sont lentement variables à l'échelle de la cellule unité. Cela permet de séparer les intégrations sur les fonctions u et sur 
les fonctions enveloppes. D'autre part, l'application de l'opérateur $\mathrm{p}$, une dérivation, à un produit donne deux termes. Il en découle que :

$$
\left.\mathrm{H}_{\mathrm{er}} \propto\left\langle\psi_{\mathrm{f}}|\mathbf{A} \cdot \mathbf{p}| \Psi_{\mathrm{i}}\right\rangle=\sum_{\mathrm{d}=\mathbf{x}, \mathbf{y}, \mathbf{z}} \mid\left\langle\mathrm{F}_{\mathrm{f}}\left|\mathbf{A}_{\mathrm{d}} \mathrm{p}_{\mathrm{d}}\right| \mathrm{F}_{\mathrm{i}}\right\rangle\left\langle\mathbf{u}_{\mathrm{f}} \mid \mathbf{u}_{\mathrm{i}}\right\rangle+\left\langle\mathrm{F}_{\mathrm{f}} \mid \mathrm{F}_{\mathrm{i}}\right\rangle\left\langle\mathrm{u}_{\mathrm{f}}\left|\mathbf{A}_{\mathrm{d}} \mathrm{p}_{\mathrm{d}}\right| \mathbf{u}_{\mathrm{i}}\right\rangle\right]
$$

où $\mathrm{d}$ repère la direction de la polarisation.

Nous allons maintenant discuter ces deux termes séparément.

\subsubsection{Transitions intrabandes}

Dans le premier terme du hamiltonien d'interaction, c'est la fonction enveloppe qui est dérivée. Le produit scalaire $\left\langle u_{f} \mid u_{i}\right\rangle$ n'est non nul que si $u_{i}=u_{f}$, puisque ces fonctions forment une base orthonormée. Ce terme correspond donc à des transitions intrabandes, c'està-dire entre un état plein et un état vide de la même bande. Cela ne peut se trouver que dans un matériau dopé.

Il y a aussi dans ce terme une intégrale sur les fonctions enveloppes, modulées par la variation exponentielle du champ:dans un matériau massif, il s'agit du terme

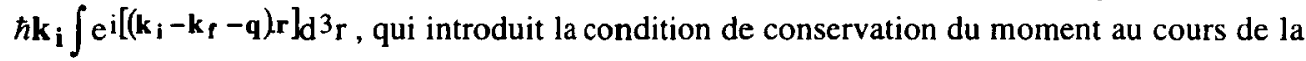
transition : $\mathbf{k}_{\mathbf{i}}-\mathbf{k}_{\mathbf{f}}=\mathbf{q}$, le (très petit) vecteur d'onde du champ. Cette condition ne peut, en pratique, être satisfaite à l'intérieur d'une même bande, à cause de la très grande pente de la relation de dispersion de la lumière. De fait, ce qu'on appelle "l'absorption due aux porteurs libres" dans un semi-conducteur dopé n'est possible qu'en faisant intervenir des processus supplémentaires de relaxation du moment (phonons). Elle est donc intimement reliée à la conductivité (YC p.296).

Par contre, si l'un des états considéré n'a plus la forme de Bloch, il est possible d'envisager de telles transitions intrabandes directes. C'est le cas à partir d'un état lié d'impureté, d'intérêt surtout académique. C'est surtout vrai dans les hétérostructures, qui ont ouvert, fondées sur ce principe, de nouvelles possibilités d'études et de dispositifs optiques dans le domaine de l'infrarouge moyen. J'y reviendrai plus loin en détail au paragraphe 4.9.

\subsubsection{Transitions interbandes}

Dans le second terme de l'expression (4.2), la dérivation s'applique aux fonctions $\mathrm{u}$. Il y a donc possibilité de changer de bande dans la transition : les énergies mises en jeu sont plutôt proches du gap, ou supérieures, et les transitions interbandes peuvent concerner l'optique du proche infrarouge et du visible.

De fait, l'élément de matrice $\left\langle u_{\mathrm{f}}\left|\mathrm{p}_{\mathrm{d}}\right| \mathrm{u}_{\mathrm{i}}\right\rangle$, qui joue un rôle central dans la théorie des transitions optiques interbandes, est le même qui intervient dans la théorie de perturbation de Kane de la structure de bande (Cf. parag. 3.3.2). Cela renforce l'intérêt d'exprimer les états de bande dans l'esprit de cette théorie. L'étude de cet élément de matrice, conduit à une première série de règles de sélection présentées au paragraphe 4.2.1.

Dans ce terme, vient aussi le produit scalaire des fonctions enveloppes modulées par le champ. Dans un semi-conducteur massif c'est $\left.\int e^{i\left[\left(k_{i}-k_{f}-q\right)\right.} \mathbf{r}\right]_{d}{ }^{3} r$ qui exprime donc la conservation du moment. Avec, en pratique, $q=0$, on parle de transition optique verticale, et 
on fonde la notion de matériau à gap direct (Cf. parag. 2.3). Mais lorsque l'invariance de translation est brisée, et particulièrement dans les hétérostructures, ce produit scalaire introduit de nouvelles règles de sélection, comme on va le voir au paragraphe 4.2.2.

\subsection{Transitions interbandes : règles de sélection et premiers effets du confinement}

Nous allons donc discuter ici successivement les deux éléments du terme interbande de la force de transition, et aussi l'influence sur les propriétés optiques de la densité en énergie des états électroniques.

\subsubsection{L'élément de matrice interbande}

$$
\begin{gathered}
<s \mid X>= \\
s_{\mathrm{AL}}+2
\end{gathered}
$$

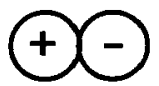

multiplié par

$$
<S\left|P_{X}\right| X>=
$$

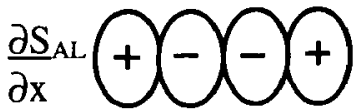

multiplié par

$$
<S\left|P_{Y}\right| X>=
$$

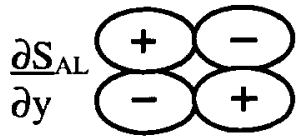

multiplié par

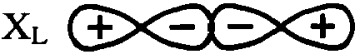

$=0$

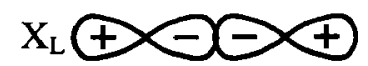

$$
=\mathbf{P}, \neq 0
$$

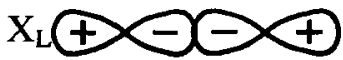

$=0$

Figure 4.1 : Essai de représentation des éléments de matrice de l'opérateur $\mathbf{p}=-i \hbar \vec{\nabla}$, une dérivation, entre bandes de valence ( $X$ liant) et de conduction ( $S$ antiliant).

Il s'agit ici d'expliciter le calcul de l'élément de matrice les matériaux camme GaAs, CdTe, etc., matériaux, on l'a vu au paragraphe 1, les plus intéressants pour nous. Je rappelle qu'ils cristallisent dans la structure cubique blende de zinc; au centre de la zone de Brillouin, la bande de conduction est issue des états $S$, et la bande de valence des états $P(X, Y, Z)$. Nous avons déjà écrit les états "atomiques" de départ pour expliciter notre approche en liaisons fortes (fig. 2.2). Rappelons nous qu'ils sont orthogonaux et que l'opérateur $p$ est une dérivation. On se convainc alors facilement en s'aidant de la figure 4.1 que seuls sont non nuls les éléments de type : $\left\langle S\left|p_{x}\right| X\right\rangle=\left\langle S\left|p_{y}\right| Y\right\rangle=\left\langle S\left|p_{z}\right| Z\right\rangle=i P$, où $P$ est l'élément de matrice de Kane. Bien entendu, cette approche très qualitative est totalement confortée par la théorie des groupes (YC p.41). Donc un champ polarisé le long de $\mathrm{x}$ ne couplera à la bande de conduction 
que la partie $\mathrm{X}$ des états de valence. Je rappelle que $\mathrm{P}$ a pratiquement la même valeur dans tous les "bons" semi-conducteurs: $\mathrm{P}^{2} / \mathrm{m}_{0}=10 \mathrm{eV}$.

Plusieurs conséquences peuvent être évoquées ici, dans les matériaux massifs et les hétérostructures.

Considérons d'abord l'excitation à travers le gap de paires électron-trou, d'énergie assez grande pour que les vecteurs d'onde des porteurs ne soient pas négligeables. Les électrons photocréés ont un moment $\mathbf{k}$ dont la direction prédominante est perpendiculaire à la polarisation du champ électrique, alors que ceux photocréés à partir de la bande de trous légers ont un moment plutôt parallèle au champ. I suffit pour le comprendre de se référer sur la figure 2.2 à la forme des fonctions d'onde $\mathrm{m}= \pm 3 / 2$ (trous lourds) et $\mathrm{m}= \pm 1 / 2$ (trous légers), et se rappeler ( $\mathrm{Cf}$. parag. 2.2.3.3) que le moment orbital des trous est quantifié le long de la direction de $\mathbf{k}$ dans un matériau massif.

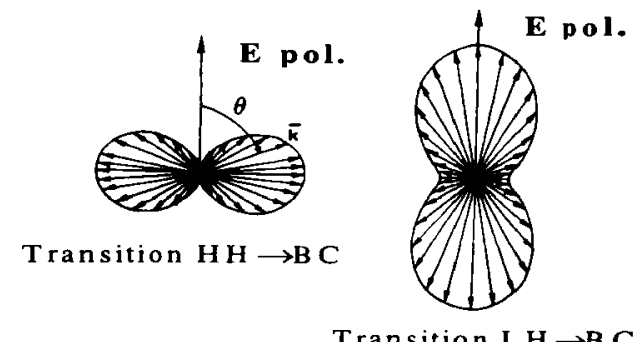

Transition $\mathbf{L ~} \mathbf{H} \rightarrow \mathrm{B} \mathbf{C}$

Figure 4.2 : Distribution du moment $k$ des électrons photocréés à une énergie non négligeable. La longueur d'un vecteur donné est proportionnelle à la population des états dont le moment $\mathbf{k}$ est parallèle à ce vecteur. D'après F. Charfi [33]

La répartition des électrons de conduction dans l'espace des $\mathbf{k}$ portera donc la trace de la polarisation (Cf. fig.4.2), du moins avant relaxation du moment. C'est l'alignement optique des électrons [33,34]. La luminescence hors d'équilibre, mais aussi de nombreux effets d'optique non linéaire (saturation d'absorption par exemple), gardent la mémoire de cet alignement.

Voici une autre conséquence dans une hétérostructure bidimensionnelle. La dégénérescence trou-lourd/trou léger est levée, y compris en $\mathbf{k}_{\perp}=0$, où le moment orbital est quantifié le long de l'axe de croissance, $z$. On constate alors facilement que les transitions impliquant les trous lourds sont interdites en polarisation $/ / \mathrm{z}$.

Enfin, en explicitant les éléments de matrice de p pour les diverses polarisations, on montre aussi comment réaliser l'orientation de spin par pompage optique. Cette technique fera l'objet d'un développement plus long au paragraphe 4.8 .

\subsubsection{Le recouvrement des fonctions enveloppes}

On va maintenant voir comment évolue la règle de conservation du moment $\mathbf{k}_{\mathbf{i}} \approx \mathbf{k}_{\mathbf{f}}$ hors du matériau massif homogène.

Lorsqu'intervient dans la transition un état lié suffisamment profond, la conservation de $\mathrm{k}$ est en partie relaxée. Ainsi, la recombinaison d'un électron (de conduction) sur un accepteur neutre, est permise sur toute la largeur de la zone de Brillouin, avec une intensité variable qui reflète la composante de Fourier en $k$ de la fonction enveloppe de l'accepteur. C'est un outil souvent utilisé pour l'étude optique des distributions énergétiques d'électrons.

Dans les matériaux désordonnés (amorphes), on s'attend aussi à une relaxation de cette règle. Mais, plus important pour notre propos, dans les hétérostructures, la notion de moment perd son sens pour les directions de confinement. En conséquence, la règle des transitions verticales est partiellement remplacée par d'autres règles de sélection dépendant des fonctions enveloppes des états confinés. 
Explicitons en quelques conséquences pour les structures bidimensionnelles de type I. Dans une structure symétrique (puits quantique carré, double puits) le hamiltonien effectif étant symétrique, les fonctions enveloppes propres sont, elles, symétriques (n impair) ou antisymétriques (n pair). On en déduit la règle de sélection :

Transition permise seulement si $n_{f}-n_{i}$ est pair (pour une structure symétrique) (4.3a)

Lorsque les deux particules sont confinées dans un puits carré de barrière suffisamment haute pour que l'approximation du puits infini soit légitime (compte tenu des masses effectives), le hamiltonien effectif ne dépend plus de la masse effective de la particule. En conséquence, les fonctions enveloppes propres d'électron et de trou d'indice différent sont orthogonales :

Transition permise seulement si $n_{f}-n_{i}=0$ (dans l'approximation du puits infini) (4.3 b)

Cette approche se trouve souvent réaliste, par exemple dans le système GaAs/AlAs pour des puits assez larges, où les transitions à $\Delta \mathrm{n} \neq 0$ sont nettement moins intenses que les autres.

\subsubsection{Densités d'états conjointes}

L'absorption optique, calculée à partir des éléments de matrice de A.p par la Règle d'Or de Fermi, fait intervenir aussi la densité conjointe des états de départ et d'arrivée. Comme on l'a vu au paragraphe 3.2 , cette densité d'états dépend de la dimension du système. On montre facilement que chaque niveau apporte une densité d'états :

$\rho(E)=\left(E-E_{G}\right)^{\frac{d-2}{2}} \Theta\left(E-E_{G}\right)$

pour $d=1,2$ ou 3 . $E_{G}$ est l'énergie du niveau de la structure, c'est-à-dire inclut les effets de confinement à $\mathrm{d}<3$. La dépendance spectrale de l'absorption sera donc très différente, comme il est illustré sur la figure 4.3. Il est intéressant de noter que, sans même introduire d'effet excitonique, réduire la dimension permet de mieux "piquer" l'absorption, avec les conséquences qu'on peut imaginer sur les propriétés non linéaires.

\subsection{Excitons}

Le concept d'exciton s'impose expérimentalement dans l'étude des propriétés optiques des semi-conducteurs, surtout pour les grands gaps $(>2 \mathrm{eV})$ et dans les hétérostructures, où ses effets peuvent rester visibles à température ambiante. Il est pertinent dans le régime linéaire (faible excitation) ; de fait, nous verrons en plusieurs occasions au 
cours de cette École que les premières non-linéarités résonantes de type $\chi_{3}$ ont pour origine l'altération des effets d'excitons due à la puissance excitatrice.

Schéma à 1 particule:
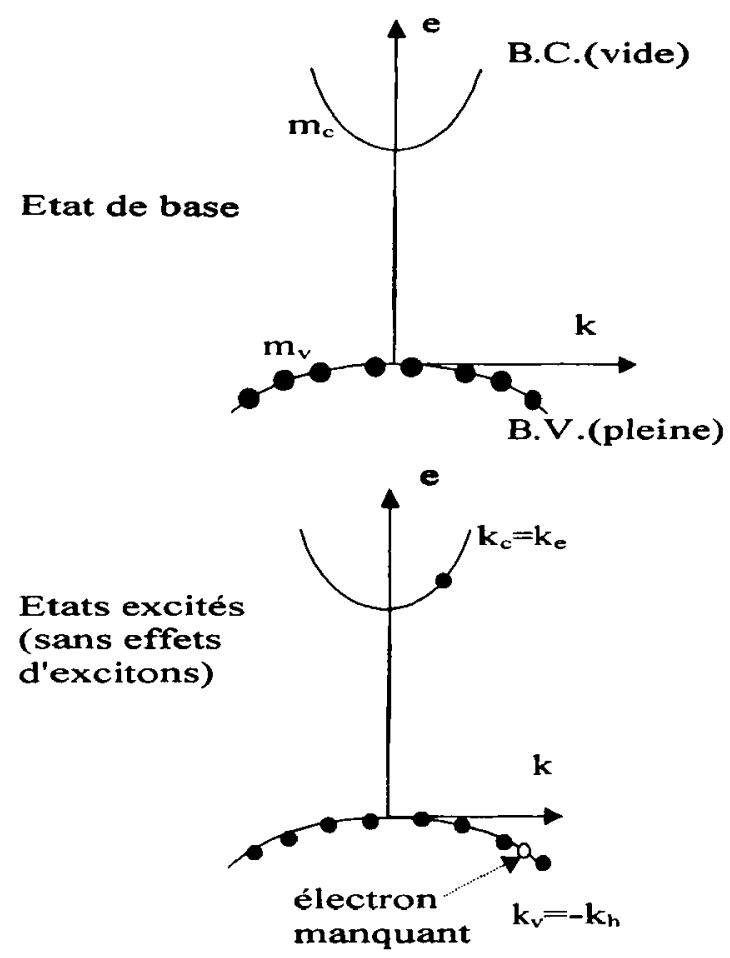

Etats excités

(avec effets d'excitons

et, à grande échelle,

effet polariton)
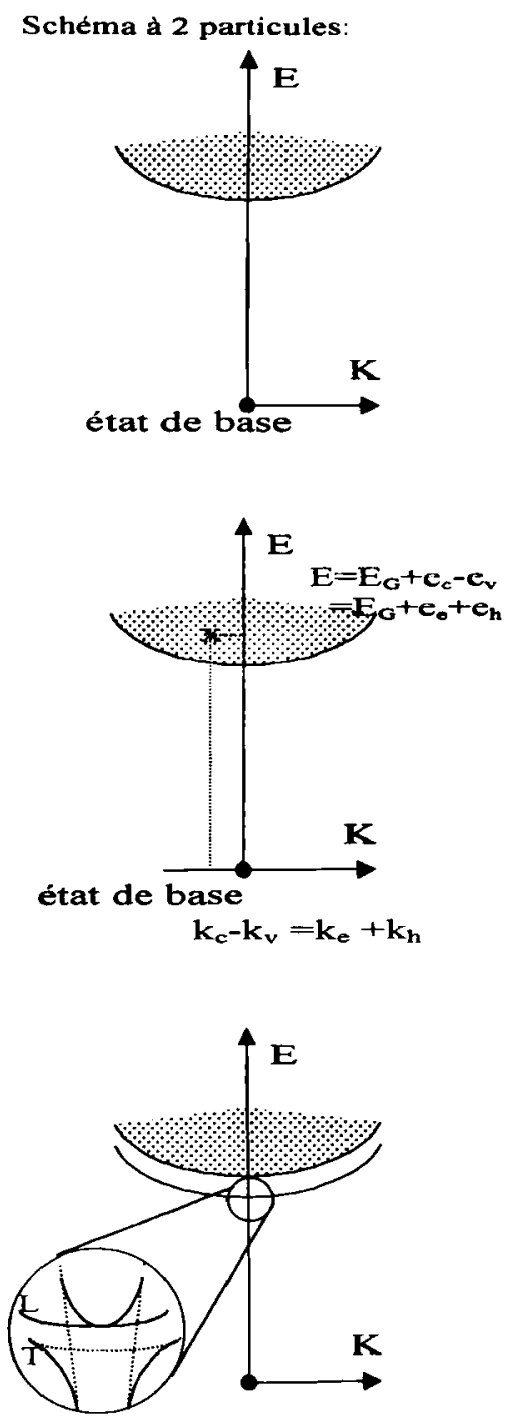

Figure 4.4 : Un schéma à 2 particules est indispensable pour comprendre l'exciton et, bien sûr, le polariton excitonique. En haut, l'état de base est représenté par le point 0,0 . Au milieu, un état excité (paire électron-trou) par un point à l'intérieur de la parabole de courbure équivalent à une masse $M=m_{e}+m_{h}$. En bas, en incluant la

liaison électron trou, on obtient les états d'exciton en dessous de la parabole des paires électron-trou. $\grave{A}$ l'intersection avec la courbe de dispersion des photons, le « zoom » montre l'anticroisement de l'effet Polariton.

Rappelons qu'il s'agit de décrire les premières excitations élémentaires du système électronique, à nombre d'électrons constant. Cette situation physique contraste avec celle du 
dopage, où l'on modifie le nombre de particules qui peuplent les états de la structure, et qui se décrit bien sous l'approximation à un électron, tant que le dopage reste moyen. Dans ce dernier cadre, les excitations élémentaires du système sont les "paires électron-trou", qui fournissent une description satisfaisante de la conductivité intrinsèque, et même de l'absorption optique pour les énergies assez nettement supérieures au gap. Parler d'exciton, c'est aller au-delà de cette approximation à un électron. Dans ce domaine comme dans d'autres, l'intuition physique, guidée par l'expérience, a précédé un établissement théorique rigoureux. Je m'en tiendrai (par goût et par nécessité) à l'approche la plus intuitive.

Sur les excitons, le classique, aujourd'hui très vieilli, mais restant pour certains problèmes théoriques une référence indispensable, est le livre de Knox [35]. Comme livre de revue d'avant les hétérostructures, il faut citer celui édité par E.I. Rashba et M.D. Sturge [36]. On trouvera enfin une discussion critique et récente des théories naïves comme celle présentée ici, dans une contribution de $\mathrm{M}$. Combescot [37]. Dans les hétérostructures, les excitons sont présents dans toute revue ou livre consacré aux propriétés optiques, et par exemple dans les références [4] pour la théorie, $[5,6]$ pour des résultats expérimentaux. Cependant, le récent compte rendu d'École édité par Burstein et Weisbuch [27] est sans doute aujourd'hui le plus complet.

\subsubsection{L'exciton dans l'approximation de la masse effective : une vue dans l'espace réel}

Considérons, formellement, un électron supplémentaire placé dans la bande de conduction. Les interactions entre cette particule et les $\mathrm{N}$ autres électrons de la bande de valence sont, par définition, prises en compte dans la valeur du gap. Retirer maintenant un électron dans la bande de valence va modifier ces interactions. C'est cette modification qui est approchée par une interaction coulombienne entre électron et trou. Il en résulte des états liés électron-trou qui ne peuvent plus se représenter dans un schéma de bande traditionnel, mais dans un schéma à deux particules (Cf. fig.4.4).

Cette interaction coulombienne se traite ensuite dans le cadre de l'approximation de la masse effective, avec les réserves usuelles sur sa validité : il faut que la fonction d'onde décrivant le mouvement relatif électron-trou (mouvement interne) soit délocalisée sur un grand nombre de cellules élémentaires, ce qui se vérifiera a posteriori dans tous les semiconducteurs usuels. On parle alors d'exciton de Wannier, par opposition aux excitons de Frenkel, où l'électron et le trou restent localisés ensemble autour d'une même cellule. Dans le même esprit pragmatique, on pondère l'interaction coulombienne par une constante diélectrique $\varepsilon$, dont la valeur précise dépendra de la fréquence caractéristique du mouvement interne de façon autoconsistante. En pratique, on prendra la valeur statique, sauf si le mouvement interne est plus rapide que celui des phonons optiques, ce qui se traduit par $\mathbf{R}_{\mathrm{ex}}^{*}>\hbar \omega_{\mathrm{ph} . \mathrm{LO}}$; on prendra alors la valeur de $\varepsilon$ diminuée de leur contribution.

On se limite ici au cas de deux bandes paraboliques et non dégénérées de masses effectives $m_{e}$ et $m_{h}$. La suite du raisonnement est exposée ci-dessous à 3 dimensions, mais est facilement généralisable à $2 \mathrm{D}$. Une écriture générale de fonction d'onde à deux particules, particulièrement pédagogique, est obtenue sur la base des fonctions de Wannier (Cf. parag. 2.2.4.2) :

$$
\psi\left(\mathbf{r}_{\mathbf{e}}, \mathbf{r}_{\mathbf{h}}\right)=\mathbf{N}^{-1} \sum_{\mathbf{R}_{\mathbf{e}}, \mathbf{R}_{\mathbf{h}}} \Phi\left(\mathbf{R}_{\mathbf{e}}, \mathbf{R}_{\mathbf{h}}\right) \mathbf{w}\left(\mathbf{r}_{\mathbf{e}}\right) \mathbf{w}\left(\mathbf{r}_{\mathbf{h}}\right)
$$


où $\mathbf{N}$ est le nombre de sites (facteur de normalisation). Surtout, $\mathbf{r}_{\mathbf{e}}$ et $\mathbf{r}_{\mathbf{h}}$ sont les coordonnées de l'électron et du trou dont on veut décrire le mouvement, alors que $\mathbf{R}_{\mathbf{e}}$ et $\mathbf{R}_{\mathbf{h}}$ sont les sites qui labellent les fonctions de Wannier, localisées. $\Phi\left(\mathbf{R}_{\mathbf{e}}, \mathbf{R}_{\mathbf{h}}\right)$ est la fonction enveloppe à deux particules sur laquelle nous allons travailler maintenant.

L'esprit de l'EFA consiste à user des coordonnées des sites comme s'il s'agissait de variables continues. Le hamiltonien effectif du système électron-trou sera de même type que celui de l'atome d'hydrogène, avec bien sûr, des masses effectives affectant le terme cinétique et une constante diélectrique diminuant l'interaction coulombienne :

$$
\left[\frac{-\hbar^{2}}{2 \mathrm{~m}_{\mathrm{e}}} \nabla_{\mathbf{R}_{\mathbf{e}}}^{2}-\frac{\hbar^{2}}{2 \mathrm{~m}_{\mathrm{h}}} \nabla_{\mathbf{R}_{\mathbf{h}}}^{2}-\frac{\mathrm{e}^{2}}{4 \pi \varepsilon_{0} \varepsilon\left|\mathbf{R}_{\mathbf{e}}-\mathbf{R}_{\mathbf{h}}\right|}\right] \boldsymbol{\Phi}\left(\mathbf{R}_{\mathbf{e}}, \mathbf{R}_{\mathbf{h}}\right)=\mathbf{E}_{\mathbf{x}} \boldsymbol{\Phi}\left(\mathbf{R}_{\mathbf{e}}, \mathbf{R}_{\mathbf{h}}\right)
$$

qui se traite comme il est bien connu dans le problème classique, en séparant et quantifiant successivement le mouvement du centre de masse d'une part, le mouvement relatif (interne) d'autre part :

$$
\left[\frac{-\hbar^{2}}{2 M} \nabla_{\mathbf{R}}^{2}\right] \mathrm{F}(\mathbf{R})=\mathrm{E}_{\mathbf{R}} \mathrm{F}(\mathbf{R}) \text { pour les coordonnées } \mathbf{R}=\left(\mathrm{m}_{\mathrm{e}} \mathbf{r}_{\mathbf{e}}+\mathrm{m}_{\mathbf{h}} \mathbf{r}_{\mathbf{h}}\right) / \mathrm{M} \text { du centre de }
$$
masse, avec $M=m_{e}+m_{h}$, la masse totale , et :

$$
\left[\frac{-\hbar^{2}}{2 \mu} \nabla_{\mathbf{r}}^{2}-\frac{\mathrm{e}^{2}}{4 \pi \varepsilon_{0} \varepsilon \mathbf{r}}\right] \varphi(\mathbf{r})=\mathrm{E}_{\mathbf{r}} \varphi(\mathbf{r}) \text { pour la coordonnée } \mathbf{r}=\mathbf{r}_{\mathbf{e}}-\mathbf{r}_{\mathbf{h}} \text { du mouvement }
$$

interne, avec $\mu=\left(\mathrm{m}_{\mathrm{e}}^{-1}+\mathrm{m}_{\mathrm{h}}^{-1}\right)^{-1}$ la masse réduite.

Ces deux mouvements sont découplés, les états propres sont produits d'ondes planes pour $\mathbf{R}$ : $F(R)=N^{-1 / 2}$ eiK.R d'énergie $E_{R}=\hbar^{2} K^{2} / 2 M$, et de fonctions hydrogénoïdes pour $r$. Il sont donc caractérisés par l'indice $n(=1 s, 2 s, 2 p, \ldots)$ du mouvement interne et le vecteur d'onde $\mathbf{K}$ du mouvement d'ensemble.

Tableau 4.1 : Résumé des principales propriétés des états d'excitons à 3 et 2 dimensions, en fonction du

\begin{tabular}{|c|c|c|}
\hline & $3 \mathrm{D}$ & $2 \mathrm{D}$ \\
\hline Energie de liaison & $\begin{array}{l}\mathrm{R}^{*} 1 / \mathrm{n}^{2} \\
\mathrm{n}=1,2, \text { etc... }\end{array}$ & $\begin{array}{l}\mathrm{R}^{*} 1 /(\mathrm{n}+1 / 2)^{2} \\
\mathrm{n}=0,1, \text { etc } \ldots\end{array}$ \\
\hline $\begin{array}{l}\text { Fonction enveloppe radiale } \\
\text { de l'état de base, }\end{array}$ & $\varphi_{1,0}=\mathrm{a}^{*-3 / 2} 2 \mathrm{e}^{-\mathrm{r} / \mathrm{a}^{*}}$ & $\varphi_{0,0}=\mathrm{a}^{*-1} 4 \mathrm{e}^{-2 \mathrm{r} / \mathrm{a}^{*}}$ \\
\hline $\begin{array}{l}\text { Fonction enveloppe radiale } \\
\text { du } 1^{\text {er }} \text { état excité } S\end{array}$ & $\varphi_{2,0}=\left(2 \mathrm{a}^{*}\right)^{-3 / 2}\left(2-\mathrm{r} / \mathrm{a}^{*}\right) \mathrm{e}^{-\mathrm{r} / 2 \mathrm{a}^{*}}$ & $\begin{array}{l}\varphi_{1,0}=\mathrm{a}^{*}-1(4 / 3 \sqrt{3}) \\
\mathrm{x}\left(1-4 \mathrm{r} / 3 \mathrm{a}^{*}\right) \mathrm{e}^{-2 \mathrm{r} / 3 \mathrm{a}^{*}}\end{array}$ \\
\hline $\begin{array}{l}\text { Fonction enveloppe radiale } \\
\text { des } 1^{\text {ers }} \text { états excités } \mathrm{P}\end{array}$ & $\varphi_{2,1}=\left(2 \mathrm{a}^{*}\right)^{-3 / 2}\left(\mathrm{r} / \mathrm{a}^{*} \sqrt{3}\right) \mathrm{e}^{-\mathrm{r} / 2 \mathrm{a}^{*}}$ & $\begin{array}{l}\varphi_{1, \pm 1}=a^{*-1}(16 / 9 \sqrt{6}) \\
\left(\mathrm{r} / \mathrm{a}^{*}\right) \mathrm{e}^{-2 \mathrm{r} / 3 \mathrm{a}^{*}}\end{array}$ \\
\hline $\begin{array}{l}\text { Force d'oscillateur } \\
\text { des états } S(n, l=0)\end{array}$ & $\propto 1 / n^{3}$ & $\propto 1 /(n+1 / 2)^{3}$ \\
\hline
\end{tabular}
Rydberg $\mathrm{R}^{*}$ et du rayon de Bohr $\mathrm{a}^{*}$ tridimensionnels, dans les matériaux de type zinc blende à transition permise en $k=0$. 
Le tableau 4.1 rappelle quelques résultats classiques sur ces fonctions à 3 et 2 dimensions. Les quantités caractéristiques de ces états s'expriment à partir d'un Rydberg effectif $R_{X}^{*}=13,6 \mathrm{eV} \frac{\mu}{m_{0}} \frac{1}{\varepsilon^{2}}$ qui vaut typiquement de 1 à $100 \mathrm{meV}$.

et d'un rayon de Bohr effectif $\mathrm{a}_{\mathrm{B}}^{*}=0.053 \mathrm{~nm} \frac{\mathrm{m}_{0}}{\mu} \varepsilon$ de 1 à $50 \mathrm{~nm}$, ce qui justifie a posteriori I'EFA pour ces excitons de Wannier. Dans beaucoup de cas, $m_{\mathrm{e}} \ll m_{h}$, et donc ces quantités sont proches de celles d'un donneur.

Il est bien entendu possible de trouver dans la littérature des calculs plus poussés, prenant en particulier en compte la dégénérescence de la bande de valence [38]. Mais il est plus important, pour notre propos, de compléter nos points de vue de la même réalité.

\subsubsection{L'exciton dans l'approximation de la masse effective : une vue dans l'espace réciproque}

Il est intéressant de ne pas s'en tenir à une représentation de l'exciton sur l'espace réel des fonctions de Wannier, mais d'en avoir une vision "transformée de Fourier" dans l'espace des k. Cela peut se faire à propos de nombreux problèmes mais il se trouve que nous aurons besoin dans la suite d'écrire les opérateurs création et annihilation d'exciton. Je suppose le lecteur familiarisé avec le formalisme de la seconde quantification. Si ce n'est pas le cas, il pourra s'initier [39] et trouvera un premier exercice d'application facile dans ce qui suit et le paragraphe 4.4.2.

Nous allons construire les opérateurs «excitons» $X_{n, K}^{+}$et $X_{n, K}$ par combinaison linéaire des opérateurs «électron » et «trou », ce qui est en principe toujours possible, ces derniers formant une base complète. Ce sont :

$\alpha_{\mathbf{k}_{\mathbf{e}}}^{+}, \alpha_{\mathbf{k}_{\mathbf{e}}}$ pour les électrons et

$\beta_{\mathbf{k}_{h}}^{+}=-\alpha_{\mathbf{k}_{h}}, \beta_{\mathbf{k}_{h}}=\alpha_{\mathbf{k}_{e}}^{+}$pour les trous.

Dans le cadre de l'EFA (appliquée sur la base des fonctions de Wannier) l'opérateur création d'exciton de niveau $\mathrm{n}$ et de vecteur d'onde $\mathrm{K}$ pourra donc s'écrire :

$$
\begin{aligned}
& \mathrm{X}_{\mathbf{n}, \mathbf{K}}^{+}=\sum_{\mathbf{k}_{\mathbf{e}}, \mathbf{k}_{\mathbf{h}}}\left\langle\mathbf{k}_{\mathbf{e}}, \mathbf{k}_{\mathbf{h}} \mid \mathrm{n}, \mathbf{K}\right\rangle \alpha_{\mathbf{k}_{\mathbf{e}}}^{+} \beta_{\mathbf{k}_{\mathbf{h}}}^{+}=
\end{aligned}
$$

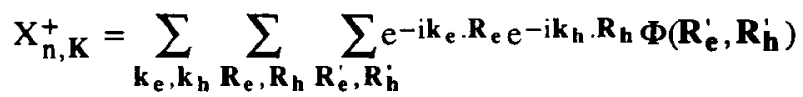

$$
\begin{aligned}
& x \int w_{\mathbf{R}_{e}}^{*}\left(\mathbf{r}_{e}\right) w_{\mathbf{R}_{h}}^{*}\left(\mathbf{r}_{h}\right) w_{\mathbf{R}_{e}^{\prime}}\left(\mathbf{r}_{\mathbf{e}}\right) w_{\mathbf{R}_{h}}\left(\mathbf{r}_{h}\right) d^{3} r_{e} d^{3} r_{h} \alpha_{\mathbf{k}_{\mathbf{e}}}^{+} \beta_{\mathbf{k}_{h}}^{+}
\end{aligned}
$$

L'expression se simplifie en utilisant le fait que les fonctions de Wannier forment une base orthonormée, ce qui introduit les conditions $\mathbf{R}_{\mathbf{e}}^{\prime}=\mathbf{R}_{\mathbf{e}}$ et $\mathbf{R}_{\mathbf{h}}=\mathbf{R}_{\mathbf{h}}$. Ensuite, les choses s'éclairent avec le changement de variable :

$$
\begin{aligned}
& \overline{\mathbf{K}}=\mathbf{k}_{\mathbf{e}}+\mathbf{k}_{\mathbf{h}} \quad \mathbf{R}=\left(\mathrm{m}_{\mathrm{e}} \mathbf{r}_{\mathbf{e}}+\mathrm{m}_{\mathrm{h}} \mathbf{r}_{\mathbf{h}}\right) / \mathrm{M} \\
& \overline{\mathbf{k}}=\left(\frac{\mathbf{k}_{\mathbf{e}}}{\mathrm{m}_{\mathrm{e}}}-\frac{\mathbf{k}_{\mathbf{h}}}{\mathrm{m}_{\mathrm{h}}}\right) \frac{\mathrm{m}_{\mathrm{e}} \mathrm{m}_{\mathrm{h}}}{\mathrm{M}} \quad \mathbf{r}=\mathbf{r}_{\mathbf{e}}-\mathbf{r}_{\mathbf{h}}
\end{aligned}
$$


Comme attendu, on va traiter les coordonnées de site comme des variables continues, transformant les sommes en intégrales avec les coefficients de normalisation ad hoc. Alors, l'expression (4.5) s'écrit sans trop de difficultés [40] :

$$
X_{n, K}^{+}=\sum_{\overline{\mathbf{k}}} \bar{\varphi}(\overline{\mathbf{k}}) \alpha_{\frac{m_{e}}{M} \mathbf{K}+\overline{\mathbf{k}}}^{+} \beta_{\frac{m_{h}}{M} K-\overline{\mathbf{k}}}^{+}
$$

L'état d'exciton est construit, on s'en doutait, sur des paires électron-trou de même $\overline{\mathbf{K}}=\mathbf{k}_{\mathbf{e}}+\mathbf{k}_{\mathbf{h}}=\mathbf{K}$. Le deuxième vecteur caractéristique de la paire, $\overline{\mathbf{k}}$, se distribue sur tout l'espace réciproque, mais pondéré par la fonction $\vec{\varphi}(\overline{\mathbf{k}})$, transformée de Fourier de la fonction hydrogénoïde $\varphi(\mathbf{r})$.

Pour les états de base de l'exciton, on a

en $3 d: \vec{\varphi}(\overline{\mathbf{k}})=8 \sqrt{\pi \mathrm{a}_{\mathrm{B}}^{*}}\left[1+\left(\mathrm{ka}_{\mathrm{B}}^{*}\right)^{2}\right]^{-2}$, et en $2 \mathrm{~d}: \vec{\varphi}(\overline{\mathbf{k}})=\sqrt{2 \pi} \mathrm{a}_{\mathrm{B}}^{*}\left[1+\left(\mathrm{ka}_{\mathrm{B}}^{*} / 2\right)^{2}\right]^{-3 / 2}$

L'exciton est donc construit essentiellement sur des paires électron-trou de $\overline{\overline{\mathbf{k}}}$ inférieur à l'inverse du rayon de Bohr, avec une probabilité maximum pour celles de $\overline{\overline{\mathbf{k}}} \approx \mathbf{0}$. Il est intéressant de remarquer, en utilisant (4.7) et l'approximation des bandes paraboliques, que :

$$
\left.\overline{\mathbf{k}} \approx \mathbf{0} \Rightarrow \frac{\mathrm{k}_{\mathrm{e}}}{\mathrm{m}_{\mathrm{e}}} \approx \frac{\mathrm{k}_{\mathrm{h}}}{\mathrm{m}_{\mathrm{e}}} \Rightarrow \frac{\partial \mathrm{E}_{\mathrm{e}}}{\partial \mathrm{k}_{\mathrm{e}}} \approx \frac{\partial \mathrm{E}_{\mathrm{h}}}{\partial \mathrm{k}_{\mathrm{h}}} \Rightarrow \frac{\partial \mathrm{E}_{\mathrm{X}}}{\partial \overline{\mathrm{k}}}\right)_{\overline{\mathrm{K}} \approx \mathrm{K}}
$$

On en déduit que :

- les paires les plus probables dans l'exciton sont celles d'énergie minimum pour un $\mathrm{K}$ donné, ce qui est logique puisqu'on cherche dans l'exciton l'état de base des états excités.

- les vitesses de groupe d'électron et de trou sont approximativement égales dans l'exciton, ce qui peut s'interpréter en disant que les deux particules "voyagent ensemble" à travers le cristal. J'ai choisi dans la figure 4.4 de représenter une telle paire, d'énergie relativement basse, et on pourra y vérifier que les pentes des courbes de dispersion pour l'électron et le trou sont proches.

L'analogie entre l'état d'exciton et un atome hydrogénoöde ne doit pas inspirer d'idée fausse. Cet état préexiste à toute illumination, mais il est vide. L'absorption d'un photon le peuple. Les analogues des états de base et excité d'un atome sont plutôt, rappelons le, les bandes de valence et de conduction. Mais l'exciton est une excitation élémentaire en théorie parfaitement délocalisée sur tout le cristal, et en pratique sur plusieurs centaines de nm, plusieurs expériences l'attestent [41].

\subsection{Vers les fortes excitations}

L'influence de la densité d'excitation sur l'intensité du pic exciton et sur sa position est à l'origine des principales non-linéarités aux énergies proches du gap, du moins dans les semiconducteurs intrinsèques. Elle relève des théories les plus élaborées de la physique des solides, celles qui permettent d'aller au-delà de l'approximation à un électron, vers les problèmes "à N-corps". Sur ce sujet immense [42], je limiterai mon ambition à donner au 
lecteur une idée des mécanismes en jeu. En simplifiant donc à l'extrême [43], je distinguerai trois types d'effets.

4.4.1 L'écrantage de l'interaction coulombienne, dû aux porteurs libres. Il est donc plutôt efficace à haute température. Par ailleurs, en 2D, ce mécanisme perd de l'efficacité car il n'est pas facile d'écranter des lignes de champ 3D avec un gaz d'électrons 2D.

On peut le décrire en modifiant l'interaction coulombienne comme suit :

$$
\frac{1}{4 \pi \varepsilon_{0} \varepsilon\left|r_{e}-r_{h}\right|} e^{\left|r_{e}-r_{h}\right| / l_{s}}
$$

La longueur $l_{s}$ peut être estimée à partir

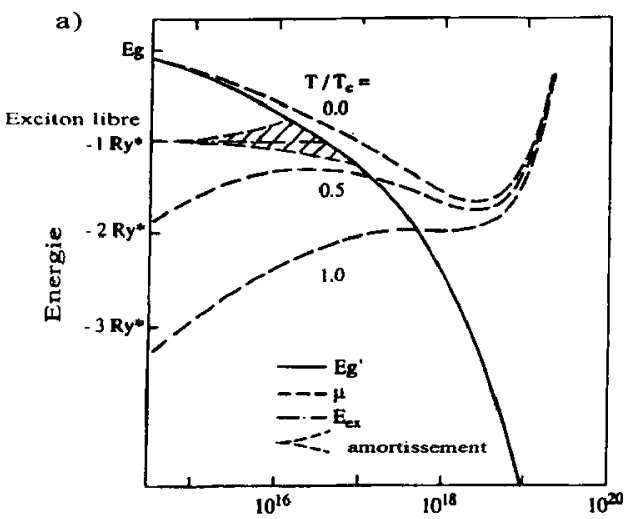

densité de paires électron-trou $\mathrm{n}_{\mathrm{p}}\left(\mathrm{cm}^{-3}\right)$

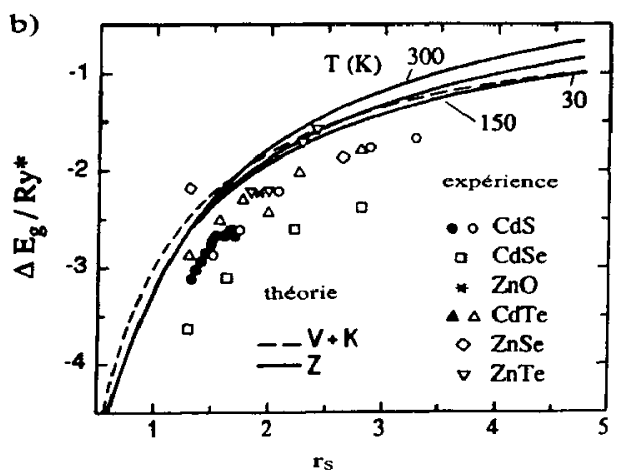

Figure 4.5 : a) Evolution, avec la densité de porteurs, de la liaison excitonique dans CdS. b) Exprimée en fonction de $r_{S}$ (voir paragraphe 4.4.3) et exprimée en unité de Rydberg Ry*, la renormalisation du gap est à peu près la même pour tous les semi-conducteurs III$\mathrm{V}$ (dont les points représentatifs se confondent avec les courbes théoriques) et II-VI. (Emprunté à C.F. Klingshirn [5] p.308) de la fonction diélectrique du gaz d'électrons $\boldsymbol{\varepsilon}(\mathbf{q})$, dépendant du vecteur d'onde. Grosso modo, elle dépend de la capacité du système à fournir des électrons pour "gommer" les variations du potentiel. La quantité clé est donc la dérivée de la densité de particules par rapport au potentiel chimique, ou niveau de Fermi : $\partial \mathrm{n} / \partial \mathrm{E}_{\mathrm{F}}$.

Pour un gaz classique (modèle de Debye Hüickel en 3D), c'est la température qui limitera l'écrantage : $1_{\mathrm{s}}^{\mathrm{DH}}=\left(\varepsilon_{0} \varepsilon \mathrm{kT} / \mathrm{e}^{2} \mathrm{n}\right)^{1 / 2}$ Pour un gaz dégénéré (modèle de Thomas Fermi, également à 3D), ce sont plutôt le principe de Pauli et la masse effective : $1_{S}^{\mathrm{TF}}=\left(2 \varepsilon_{0} \varepsilon E_{\mathrm{F}} / 3 \mathrm{e}^{2} \mathrm{n}\right)^{1 / 2}$ avec $\mathrm{n}$ relié au niveau de Fermi par $\mathrm{n}=\frac{1}{3 \pi^{2}}\left(2 \mathrm{mE}_{\mathrm{F}} / \hbar^{2}\right)^{3 / 2}$.

En pratique, à basse température et à $3 \mathrm{D}$, l'écrantage de Thomas Fermi est très efficace, et $l_{S}$ est de l'ordre de la distance moyenne entre électrons. Puis, comme la température augmente, l'écrantage de Debye prend le relais quand $k T>2 / 3 \quad E_{F}$. On le comprend, l'exciton cessera d'être lié lorsque la longueur d'écran $l_{s}$ sera comparable au rayon de Bohr $a_{B}^{*}$ de l'exciton. Cette condition peut se traduire en termes de densité critique, dite de Mott, pour la transition de phase entre un gaz d'exciton et un plasma électron-trou (Cf. fig. 4.5 a) 
4.4.2 Le remplissage de l'espace des phases s'impose donc surtout à $2 \mathrm{D}$. On dit souvent, non sans prendre un air mystérieux, que l'exciton est un boson. Mais il est composite, formé de deux fermions, l'électron et le trou. Que faut-il en penser? Nous sommes en mesure de calculer les commutateurs de type : $\left\lfloor\mathrm{X}_{1,0}, \mathrm{X}_{1,0}^{+}\right\rfloor$, qui vaut 1 pour les bosons et -1 pour les fermions. Pour simplifier, on prendra l'état de base $n=1$ et $\mathbf{K}=\mathbf{0}$. Alors, en utilisant le résultat $(4.7)$, il vient :

$\left[\mathrm{X}_{1,0}, \mathrm{X}_{1,0}^{+}\right]=\sum_{\overline{\mathbf{k}}}\left|\varphi_{1,0}\right|^{2}\left(1-\alpha_{\frac{\mathbf{k}}{ \pm} \alpha_{\overline{\mathbf{k}}}}-\beta_{-\mathbf{k}}^{+} \beta_{-\mathbf{k}}\right) \approx 1-\mathrm{o}\left(\mathrm{na}_{\mathrm{B}}^{* 3}\right)$, qui vaut donc 1 à faible densité, mais qui diminue à l'approche de la densité critique $\mathrm{a}_{\mathrm{B}}^{*-3}$. Elle n'est pas très éloignée de la densité de Mott. Autrement dit, les excitons sont des bosons tant qu'il n'y en a pas trop, c'està-dire nettement moins d'un par "volume" excitonique.

4.4.3 Ces deux phénomènes, écrantage et remplissage, se conjuguent pour faire apparaître aux excitations intermédiaires $\left(10^{16}-10^{17}\right.$ paires par $\left.\mathrm{cm}^{3}\right)$, un plasma électron-trou qui remplace le gaz d'excitons neutres. De ce fait, l'intensité du pic d'absorption caractéristique de l'exciton et son énergie de liaison diminuent. Cela tendrait à faire remonter l'énergie du gap effectif si ce mouvement n'était contrarié par un troisième phénomène important : la renormalisation du gap. On peut voir ce phénomène comme une généralisation à haute densité de la liaison excitonique, qui serait une sorte de "renormalisation locale". C'est pourquoi il est pertinent d'exprimer dans l'étude de ce phénomène :

- la densité par le paramètre réduit $\mathrm{r}_{\mathrm{s}} / \mathrm{a}_{\mathrm{B}}^{*}$, avec $4 \pi \mathrm{r}_{\mathrm{s}}^{3} / 3=\mathrm{n}$

- la fermeture du gap en unités de Rydberg excitonique $\mathrm{R}^{*}$.

Exprimée en fonction de ces quantités, la renormalisation est à peu près indépendante du matériau, du moins en 3D, comme le montre la figure $4.5 \mathrm{~b}$.

\subsection{L'exciton dans les hétérostructures}

Comme on l'a vu au paragraphe 4.3, l'exciton $1 \mathrm{~S}$ à 2 dimensions (mais l'interaction de Coulomb reste tridimensionnelle) est 4 fois plus lié qu'à 3D, toutes choses égales par ailleurs. Ce résultat n'est rigoureusement valable que pour des puits quantiques assez étroits et de barrières assez hautes pour que les états électroniques de départ soient bien bidimensionnels.

On peut interpréter cette grande énergie de liaison comme une forte tendance de l'exciton à rester sphérique malgré le confinement des barrières, pour minimiser l'énergie cinétique du mouvement interne; en conséquence, l'électron et le trou se rapprochent dans toutes les directions, et pas seulement le long de z. On peut aussi dire qu'à cause de la densité d'état en marche d'escalier, un plus grand nombre d'états de paires de basse énergie peuvent participer à la construction de l'état de base. Par contre, les états excités sont remarquablement moins liés qu'en 3D, et moins couplés à la lumière, ce qui va de pair.

Tout se passe donc comme si une fraction encore plus importante de force d'oscillateur répartie dans les bandes était regroupée sur un seul niveau. En pratique, même si la situation physique n'est pas idéalement bidimensionnelle, on observe des effets excitoniques à température ambiante, y compris dans les semi-conducteurs III-V. Cette situation conduit à un accident d'absorption ou d'indice de réfraction assez marqué, et ceci dans la matière 
condensée, c'est-à-dire avec une probabilité d'interaction forte. Elle est à l'origine du succès des hétérostructures pour l'optique non linéaire et certains dispositifs.

Parmi ces derniers, deux ont connu un développement remarquable et font l'objet de nombreuses présentations pédagogiques [44]. Entre la conception de l'effet, véritablement quantique, parfaitement descriptible dans le cadre de l'EFA, et l'introduction dans le catalogue de certains fournisseurs, il ne s'est écoulé qu'une petite décennie (1983-1993). Le premier est fondé sur l'effet Stark confiné quantiquement (QCSE) [45], où un champ électrique, parallèle à l'axe de croissance $\mathrm{z}$, sépare le long de $\mathrm{z}$ les états d'électron et de trou confinés à l'intérieur d'un puits quantique. Cela diminue l'énergie et la force de la transition excitonique, réalisant un modulateur de lumière efficace et rapide. Le second est fondé sur l'effet Wannier Stark [46]. La structure de départ est un super-réseau, présentant donc des états délocalisés le long de $z$, et construits par combinaison linéaire des états de puits couplés (Cf. parag. 3.2.1). L'application d'un champ électrique le long de $z$ lève la dégénérescence entre ces états de puits et donc diminue le couplage. On arrive au résultat paradoxal que le champ localise les états, modifiant là aussi le spectre d'absorption. Cet effet, qui s'est révélé subtilement relié à l'oscillateur de Bloch [47], est un exemple remarquable d'exercice de mécanique quantique, profond mais simple et de bon goût, qui s'est transformé en réalité au laboratoire, puis dans l'industrie.

Cependant, dans une hétérostructure non strictement bidimensionnelle, un puits large ou un super-réseau par exemple, seuls des calculs assez lourds permettront d'estimer avec précision les énergies de liaison excitoniques. Ils sont souvent fondés sur une approche variationnelle. Si l'on ne peut y consacrer du temps, on en est réduit à utiliser des abaques résumant des calculs numériques [48]. Il faut aussi citer une approche phénoménologique intéressante qui définit et utilise une dimension non entière de la structure [49]. En tous cas, le paramètre clé pour ces questions est le rapport de la largeur de la structure avec le rayon de Bohr.

Enfin, je cite ici pour mémoire les boîtes quantiques, dont D. Ricard parle en détail. Selon le rapport des dimensions caractéristiques du problème (rayon de Bohr et dimensions de la boîte), ce sera l'effet d'exciton qui perturbera l'effet de confinement, ou l'inverse.

\subsection{Couplage exciton-photon}

Le premier calcul de l'interaction entre l'exciton et le champ électromagnétique de la lumière est dû à Elliott [50]. L'élément de matrice de couplage est une somme cohérente des contributions des paires électron-trou qui construisent l'exciton :

$$
\begin{aligned}
& \langle\mathbf{n}, \mathbf{K}|\mathbf{A} \cdot \mathbf{p}| \mathbf{0}\rangle \propto \sum_{\mathbf{k}_{\mathbf{e}}, \mathbf{k}_{\mathbf{h}}}\left\langle\mathrm{n}, \mathbf{K} \mid \mathbf{k}_{\mathbf{e}}, \mathbf{k}_{\mathbf{h}}\right\rangle\left\langle\mathbf{k}_{\mathbf{e}}, \mathbf{k}_{\mathbf{h}}|\mathbf{A} . \mathbf{p}| \mathbf{0}\right\rangle \\
& \propto \sum_{\mathbf{k}_{\mathbf{e}}, \mathbf{k}_{\mathbf{h}}}\left\langle\mathbf{n}, \mathbf{K} \mid \mathbf{k}_{\mathbf{e}}, \mathbf{k}_{\mathbf{h}}\right\rangle\left\langle\mathbf{k}_{\mathbf{e}}|\mathbf{A} \cdot \mathbf{p}|-\mathbf{k}_{\mathbf{h}}\right\rangle
\end{aligned}
$$

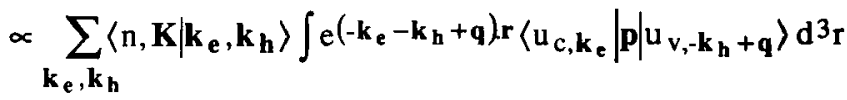

La dernière intégrale sur $r$ impose la conservation du moment : $\mathbf{k}_{\mathbf{e}}+\mathbf{k}_{\mathbf{h}}=\mathbf{q}$, le moment de la lumière. De plus, dans le cadre de l'EFA pour les excitons de Wannier, l'extension en $\mathbf{k}_{\mathbf{e}}$ et $\mathbf{k}_{\mathbf{h}}$ est réduite, ce qui permet de supposer : $\left\langle u_{c, k_{e}}|p| u_{v,-k_{h}+q}\right\rangle \approx\left\langle u_{c}|p| u_{v}\right\rangle$, indépendant de $\mathbf{k}$, et 
bien sûr de $\mathbf{q}$. La somme sur $\mathbf{k}_{\mathbf{e}}$ et $\mathbf{k}_{\mathbf{h}}$ se traite de la même façon qu'au paragraphe 4.3 .2 (éq. 4.5). En effectuant le changement de variable (4.6), il vient :

$$
\langle\mathbf{n}, \mathbf{K}|\mathbf{A} \cdot \mathbf{p}| \mathbf{0}\rangle \propto\left\langle\mathrm{u}_{\mathrm{c}}|\mathbf{p}| \mathrm{u}_{\mathrm{v}}\right\rangle \delta(\mathbf{K}-\mathbf{q}) \sum_{\overline{\mathbf{k}}} \ddot{\varphi}_{\mathrm{n}}(\mathbf{k}) \propto\left\langle\mathrm{u}_{\mathrm{c}}|\mathbf{p}| \mathrm{u}_{\mathrm{v}}\right\rangle \delta(\mathbf{K}-\mathbf{q}) \varphi_{\mathrm{n}}(\mathbf{r}=\mathbf{0})
$$

Le premier terme rappelle que l'exciton regroupe des transitions interbandes. Le second terme exprime la conservation du vecteur d'onde : l'absorption optique crée des excitons de $K=q \sim 0$. Dans le troisième terme, on n'oubliera pas que $\varphi(\mathbf{r})$ et $\vec{\varphi}(\mathbf{k})$ sont, respectivement, la fonction enveloppe des coordonnées internes à l'exciton, et sa transformée de Fourier. $\varphi_{n}(0)$ est donc la probabilité de trouver l'électron et le trou sur la même cellule unité ( "au même endroit"). Ce dernier terme n'est non nul que pour les états $n s(l=m=0)$ de l'exciton, dans le "bon" cas où l'élément de matrice interbande est non nul et indépendant de $\mathrm{k}$. Cependant, il est intéressant de noter que dans le cas où l'élément de matrice interbande est nul en $k=0$ $\left(\mathrm{Cu}_{2} \mathrm{O}\right.$ par exemple), il est possible à l'aide d'un développement limité en $\overline{\mathbf{k}}$ de montrer que les états $\mathrm{p}$ de l'exciton deviennent permis.
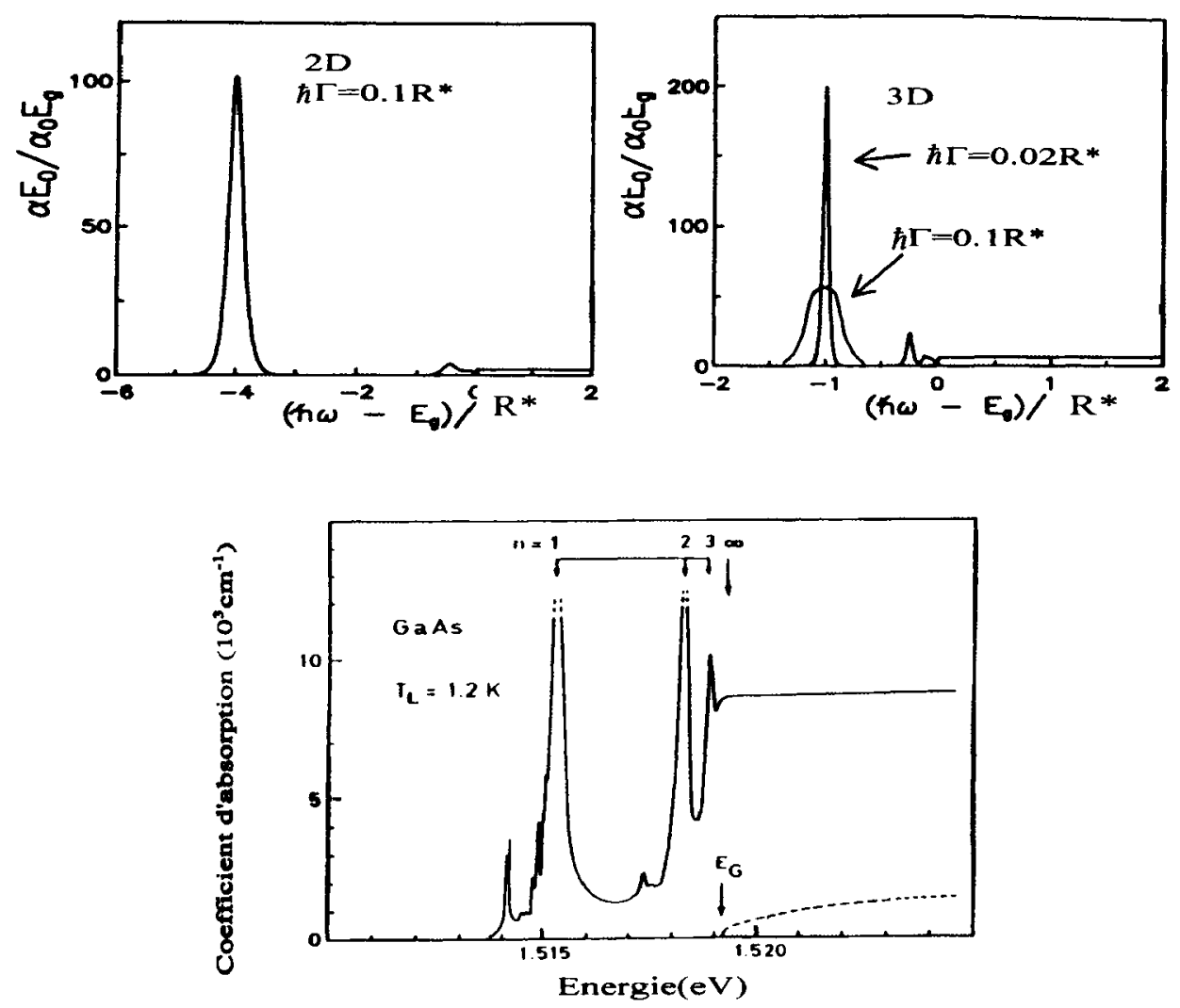

Figure 4.6 : Spectres d'absorption calcules par Haug et Koch ([4] p.196) en tenant compte de tous les effets excitoniques, y compris le facteur de Sommerfeld. On a élargi le pic exciton 3D à $0,1 R^{*}$ pour comparer à la situation 2D. En bas, magnifique spectre expérimental d'absorption de GaAs massif (3D) obtenu par R.Ulbrich ([27] p.33). En pointille, l'absorption théorique sans effet d'exciton. 
Le terme $\varphi_{n}(0)$ est proportionnel à l'inverse du "volume de Bohr". Il est donc d'autant plus fort que l'exciton est plus lié. De façon complémentaire on peut voir les paires électron-trou qui le construisent coopérer pour concentrer une part $\bar{\varphi}(\mathbf{k})$ de leur force d'oscillateur à l'énergie de l'exciton. C'est de cet effet, et non pas de l'élément de matrice de Kane, que vient le meilleur couplage exciton-photon dans les semi-conducteurs II-VI (constante diélectrique un peu plus faible et surtout grand gap, donc masse effective électronique élevée). Ce renforcement est particulièrement net à $2 \mathrm{D}$, où le premier niveau d'exciton est bien séparé des suivants (Cf. fig. 4.6).

L'influence de l'interaction coulombienne se fait également sentir sur les états non liés de l'exciton ; autrement dit, même les paires électron-trou "libres" sont corrélées positivement. II en résulte un renforcement de l'absorption au dessus du gap par rapport à la loi en $\mathrm{E}^{1 / 2}$ (à $3 \mathrm{D}$ ) ou en marche d'escalier (à 2D). Ce renforcement est souvent appelé "facteur de Sommerfeld". Il est particulièrement spectaculaire à 3D, puisque l'absorption au gap, nulle sans corrélation électron-trou, atteint la valeur qu'elle aurait à plusieurs Rydberg au dessus du gap, comme on le voit sur le spectre expérimental de la figure 4.6. À $2 \mathrm{D}$, ce renforcement au bord du gap est d'environ un facteur 2.

\subsection{Le polariton excitonique}

\subsection{1 dans les matériaux massifs}

Considérer l'absorption comme une transformation photon $\Rightarrow$ exciton, dans le schéma à deux particules de la figure 4.4 , permet de montrer qu'on a affaire à une situation physique entièrement nouvelle. En effet, avec l'exciton tridimensionnel dans son état de base, on a une excitation d'énergie bien définie, qui donne lieu à une transition optique au spectre piqué, bien que nous soyons dans un solide. Mais on ne retrouve pas la situation d'un atome, car le milieu est dense. C'est pourquoi les états sont étendus et doués d'un vecteur d'onde (les excitons). Et chaque état d'exciton est couplé à un et un seul état de photon, grâce à la règle de sélection en $\mathbf{K}$. Donc rien ne s'oppose, dans une vision première des choses, à une suite cohérente d'événements photon $\Rightarrow$ exciton $\Rightarrow$ photon $\Rightarrow$ exciton $\Rightarrow$ etc...En réalité (Cf. fig. 4.7 en haut), on a deux états discrets couplés qui vont interagir pour donner un être hybride, le polariton excitonique [51], avec un anticroisement des courbes de dispersion qu'on peut voir dans le « zoom » de la figure 4.4.

Le traitement temporel de l'interaction champ-matière est complètement différent de la situation où l'état d'arrivée de la transition appartient à un continuum, et où il y a dissipation de l'énergie dans ce continuum : c'est alors la Règle d'Or de Fermi qui décrit la transition. Cette situation est, entre autres, celle de la recombinaison excitonique d'un puits quantique placé dans un environnement quelconque ( $\mathrm{Cf}$. fig. $4.7 \mathrm{au}$ milieu).

Le polariton excitonique a de nombreux analogues en physique. Cette situation, dite de couplage fort, se retrouve depuis l'expérience d'amphithéatre des deux pendules couplés, jusqu'au polariton-phonon, qui décrit le couplage entre la lumière (infrarouge) et les oscillateurs mécaniques que sont les phonons optiques. En l'absence d'interaction dissipative supplémentaire (diffusion, collisions inélastiques avec les phonons,...), il n'y a plus d'absorption. La réflectivité, la transmission, la luminescence sont décrites comme propagation de polaritons à travers la surface de l'échantillon. 
Comme dans le cas des polaritons-phonons [52], nous sommes dans de la matière dense, donc douée d'une «constante » diélectrique dépendant de la fréquence et, secondairement, du

\section{Matériau massif}

Vecteur d'onde du centre de masse de l'exciton : $\vec{k}_{\text {exciton }}$

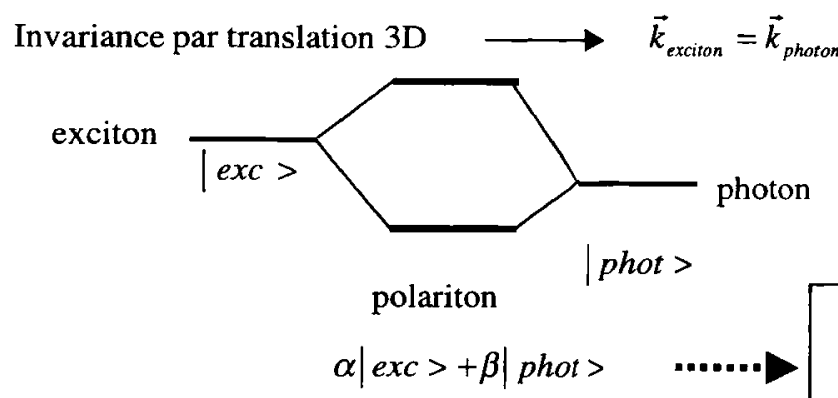

Couplage fort

Etats mixtes exciton-photon appelés polaritons

\section{Puits quantique dans un matériau massif}

Invariance par translation 2D $\longrightarrow \vec{k}_{\text {l/exciton }}=\vec{k}_{/ / \text {photon }}$

Pas de règle de sélection pour $k_{z, \text { photon }}$

exciton

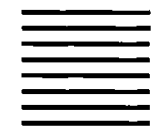

quasi-continuum de photons

\section{Couplage faible}

Règle d'Or de Fermi

Temps de vie radiatif

\section{Puits quantique dans une microcavité}

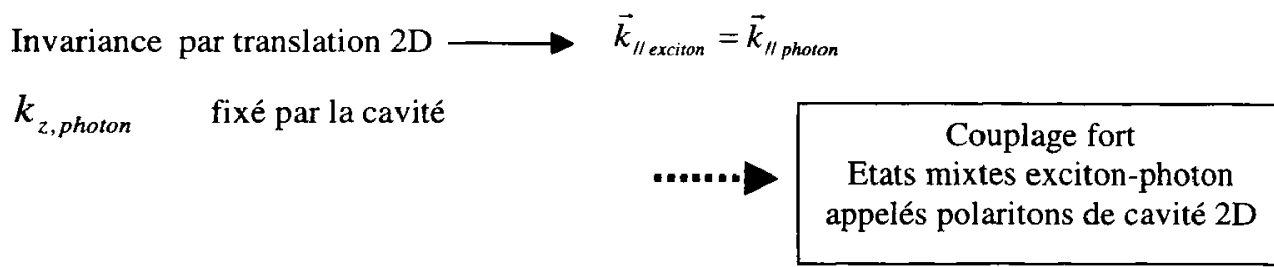

Figure 4.7 : Schéma des trois situations induites par le couplage exciton-photon. Couplage fort (en haut et en bas) lorsque les deux objets sont de même dimensionnalité : la conservation du moment induit un couplage un pour un, sans nécessiter la conservation de l'énergie. Couplage faible (au milieu) lorsque ce n'est pas le cas : la conservation de l'énergie est alors imposée par la Règle d'Or de Fermi. 
vecteur d'onde. La manipulation des équations de Maxwell permet de distinguer des ondes longitudinales et transverses. Les premières correspondent à un zéro de la fonction diélectrique et ne sont pas couplées à la lumière : $\varepsilon\left(\omega_{\mathrm{L}}\right)=0$. Les secondes correspondent à un pôle de la fonction diélectrique et se couplent à la lumière : $c^{2} \mathrm{k}^{2}=\omega_{T}^{2} \varepsilon\left(\omega_{T}\right)$. Ce couplage est intimement lié à la différence d'énergie $\Delta_{\mathrm{LT}}$ entre les ondes longitudinales et transverses. Il est donc commode d'exprimer la fonction diélectrique du système sous la forme :

$$
\varepsilon(\omega)=\varepsilon_{\text {bf }}\left(1-\frac{\Delta_{\mathrm{LT}} / \hbar}{\omega-\omega_{\mathrm{X}}(\mathrm{k})-\mathrm{i} \gamma}\right)
$$

où $\varepsilon_{b f}$ est la constante diélectrique basse fréquence, c'est-à-dire aux énergies juste inférieures à la transition considérée (elle est souvent notée $\varepsilon_{0}$, ce qui peut prêter à confusion; dans notre cas, c'est généralement la constante diélectrique statique diminuée de la contribution des phonons optiques). $\Delta_{\mathrm{LT}}$ contient pour l'essentiel la force d'oscillateur (par unité de volume) du niveau d'exciton, d'énergie $\omega_{X}(k)$. Enfin, particularité remarquable, l'énergie du pôle dépend du vecteur d'onde. C'est la "dispersion spatiale".

On tire de ces équations implicites les courbes de dispersion des ondes longitudinales et transverses, ces dernières présentant l'anticroisement qui signe le couplage fort excitonphoton. A cause de la dispersion spatiale, on trouve deux modes de $\mathrm{k}$ différents pour une même énergie au dessus de $\mathrm{E}_{\mathrm{L}}$. Il en résulte que les équations de Maxwell ne suffisent pas à décrire la transmission des polaritons à la surface du cristal. I faut y ajouter des "Conditions aux Limites Additionnelles" ( $\mathrm{ABC}$ ), qui ont été l'objet de très nombreux débats et, en pratique, dépendent de l'état de surface [53].

En résumé, la courbe de dispersion des polaritons peut n'être vue que comme la description du saut d'indice de réfraction au passage d'une transition. L'existence d'un niveau discret, lié à l'interaction excitonique, est donc vraiment essentielle, comme l'est l'aspect propagatif qui permet un couplage avec les photons.

\subsection{2 dans les hétérostructures.}

Dans les hétérostructures bidimensionnelles classiques, il faut distinguer deux situations très différentes. Imaginons pour fixer les idées un puits quantique entouré de barrières.

- il est possible d'envisager une situation analogue de couplage fort entre excitons et photons éventuellement guidés dans le plan des couches, car on y trouve un couplage un pour un. Cependant, l'interaction est nettement plus faible que dans les massifs à cause du mauvais recouvrement du champ avec l'onde de polarisation. Si l'on y ajoute les effets de désordre inévitables dans les hétérostructures, on comprend que les démonstrations expérimentales soient rares, résultant d'expériences délicates et pointues.

- par contre, pour une propagation de la lumière sortant par la surface de la structure, seule subsiste la conservation du $\mathrm{k}_{/ /}$de l'exciton (parallèle aux couches). Il n'y a donc plus de couplage un pour un et c'est la Règle d'Or de Fermi qui fixe l'énergie des photons émis. Cela vient de ce que l'espace des excitons et celui des photons n'ont plus la même dimension.

Mais on peut restaurer le couplage fort en entourant le puits d'une microstructure optique : en l'occurrence, il s'agit de réduire l'espace des photons à $\mathrm{D}=2$, ce qu'on peut faire dans une microcavité plane (Cf. fig. 4.7 en bas)

Il s'agit d'un microrésonateur Fabry-Perot. Une telle structure présente de nombreuses 
Angle externe d'émission (deg)
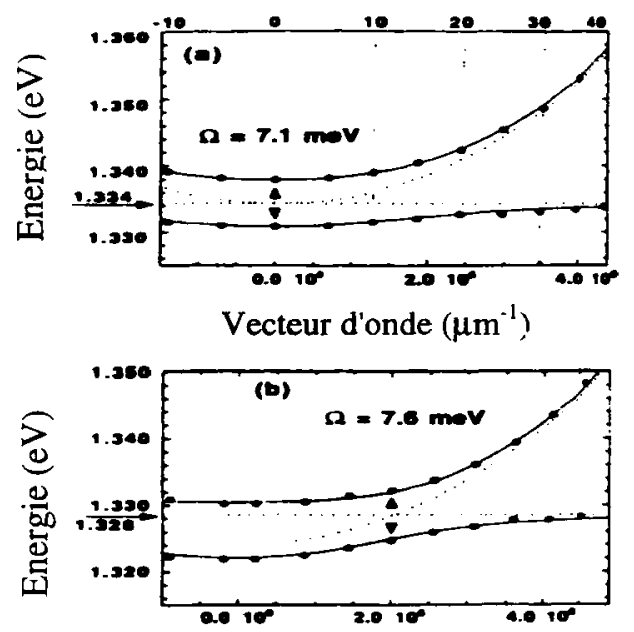

Vecteur d'onde $\left(\mu \mathrm{m}^{-1}\right)$

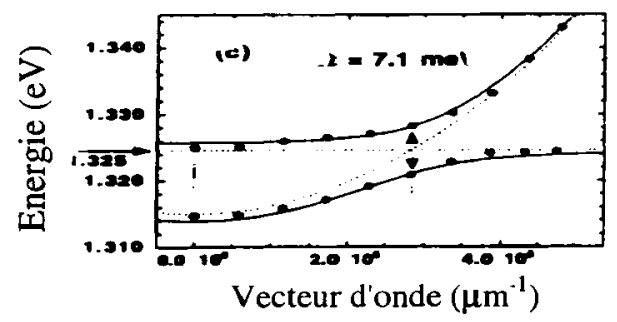

Figure 4.8 : Courbes de dispersion de polaritons de cavité mesurées par $\mathbf{R}$. Houdré [54]. L'expérience consiste à mesurer l'énergie des résonances en fonction de l'angle d'émission, associé au vecteur d'onde dans le plan. Les pointillés représentent les courbes de dispersion des photons et excitons non couplés. $\Omega$ est le « splitting de Rabi » mesuré pour chaque condition de résonance $(\mathrm{a}, \mathrm{b}$, et $\mathrm{c})$. analogies avec un «puits quantique à lumière ». Ses modes propres optiques sont caractérisés par :

- un $\mathrm{k}_{\mathrm{z}}$, fixé par la condition de résonance, $k_{Z}=k_{0}=N \pi n / L$, où $N$ est un entier, $n$ l'indice de réfraction moyen et $\mathrm{L} / \mathrm{n}$ la longueur optique de la cavité.

-un $k_{\perp}$, le rapport $k_{\perp} / k_{0}$ mesurant

l'inclinaison du mode par rapport à la normale.

Leur énergie est $E=\frac{\hbar c}{n} \sqrt{k_{0}^{2}+k_{\perp}^{2}}$ ce qui donne en première approximation une courbe de dispersion dans le plan $\mathrm{E}\left(\mathrm{k}_{\perp}\right)$ de forme parabolique.

Si l'on a maintenant inclus un puits quantique dans la cavité, la résonance avec l'état d'exciton, qui est facilement accordable, provoque un anticroisement analogue au cas tridimensionnel. Ces excitations mixtes ont été récemment découvertes et nommées "Polaritons de Cavité " [54] (Cf. fig. 4.8). Ce dédoublement (de Rabi) obtenu à la résonance peut être vu comme l'effet du saut d'indice lié à la transition sur la résonance Fabry-Perot. Il est proportionnel, typiquement, à $(\mathbf{f} / \mathrm{L})^{1 / 2}$ où $\mathbf{f}$ est la force d'oscillateur de l'exciton et $\mathrm{L}$ la longueur de cavité.

Plus généralement, ces structures offrent en optique non linéaire une exaltation des effets liée au renforcement du champ dans la cavité [55]. Elles peuvent aussi présenter des solutions élégantes au problème de l'accord de phase [56].

\subsection{Orientation et alignement par pompage optique}

Nous revenons maintenant à l'exploitation des règles de sélection interbande dues à l'élément de matrice de $\mathrm{p}$, pour considérer l'influence de la polarisation sur la nature des transitions. On s'en doute, la bonne base de départ pour les états électroniques est celle des états propres du moment total, telle que représentée sur la figure 2.2. La direction z sera choisie parallèlement à la propagation et, s'il y a lieu, de la direction de confinement. À partir des combinaisons linéaires ad hoc d'états du champ de polarisation $x$ et $y$, et des opérateurs $p_{x}$ et $p_{y}$, il est facile de définir les opérateurs $\mathrm{p}_{+}$ou $\mathrm{p}_{-}$, de les appliquer à ces états électroniques, et d'établir les probabilités de transitions résumées sur la figure 4.9. On constate que l'absorption d'un photon 
$\sigma_{+}$, d'énergie par exemple résonante avec la transition trous-lourds $\Rightarrow$ conduction et avec elle seule, transfère effectivement tout son moment angulaire à la population des électrons, en orientant le spin électronique. Bien entendu, le champ électromagnétique, à ces énergies, n'agit pas directement sur le spin. Mais l'orientation s'effectue par l'intermédiaire du couplage spin orbite. On peut par exemple vérifier que pour une excitation de grande énergie, qui rend équiprobables les transitions des trois bandes de valence, il n'y a plus de polarisation de spin de la bande de conduction. L'ensemble des sujets relatifs à l'orientation optique a fait l'objet d'articles de synthèse assez complets, mais écrits avant l'apparition des systèmes bidimensionnels [57-59].

On ne s'étonne pas que l'information portée par la polarisation de la lumière excitatrice soit, d'une façon ou d'une autre, transférée au système matériel. Ainsi des expériences non résonantes, ou bien aux temps très courts, doivent en garder trace. Mais on connaît deux circonstances où cette mémoire de polarisation est suffisamment stabilisée pour être détectée à la recombinaison, y compris en régime stationnaire (excitation quasi continue).

\subsubsection{L'orientation de spin par absorption de lumière circulairement polarisée}

Il s'agit le plus souvent de l'orientation de spin des électrons de conduction, du moins dans les matériaux massifs où, pour les états de valence, le moment de rotation est fortement couplé au moment cinétique. La moindre diffusion de $\mathrm{k}$ est alors un facteur de dépolarisation très efficace pour les trous. II suffit de lire les schémas 4.9 de haut en bas dans le sens de la recombinaison pour comprendre qu'à l'inverse, la mesure du taux de polarisation de la luminescence informe sur la polarisation résiduelle de la bande de conduction.

On peut exprimer de façon phénoménologique la polarisation d'équilibre (en excitation quasi continue) par :

$$
\mathrm{P}=\mathrm{P}_{0} \mathrm{~T}_{1} /\left(\mathrm{T}_{1}+\tau\right)
$$

où $P_{0}$ est la polarisation de création, $T_{1}$ la durée de relaxation de spin et $\tau$ la durée de vie des porteurs. En appliquant un champ magnétique transverse $\mathrm{H}$, on provoque la précession des spins, et donc une dépolarisation sous champ de la luminescence (effet Hanle) :

$$
P(H)=P(0)\left(1+\omega^{2} T_{1}^{* 2}\right)
$$

où $\omega$ est la fréquence angulaire de précession des spins, et $T_{1} *$ la durée de vie de polarisation : $\mathrm{T}_{1}^{*-1}=\mathrm{T}_{1}^{-1}+\tau$. Selon une formule fameuse [60], on peut avec cette expérience mesurer en excitation continue la durée de vie des électrons en utilisant la précession de Larmor comme une horloge.

Donnons quelques valeurs typiques de polarisation de spin qu'il est possible d'obtenir :

- L'excitation d'un matériau cubique massif au gap, avec donc trous lourds et trous légers pondérés uniquement par les probabilités d'absorption, donne une polarisation de spin, avant relaxation $P_{0}=0,5$. En supposant, ce qui est réaliste dans les matériaux massifs, que la bande de valence est complètement dépolarisée, le taux de polarisation de la luminescence est moitié de celui de la population électronique; on se trouve donc dans le cas d'une polarisation maximum de la luminescence $P_{0}^{2}=0,25$. Ce type d'expérience a été beaucoup utilisée pour étudier, précisément, la relaxation du spin et aussi de l'énergie. 
- Dans un matériau uniaxe (CdS) ou bien dans une hétérostructure bidimensionnelle, il est possible d'espérer, et d'observer, $\mathrm{P}_{0}=1$. La mémoire de polarisation de la luminescence peut donc atteindre $100 \%$ si la relaxation et la recombinaison sont rapides.

\section{POLARISATION CIRCULAIRE}

\section{Schéma à une particule}

Moment

total :

B.C.

B.v.

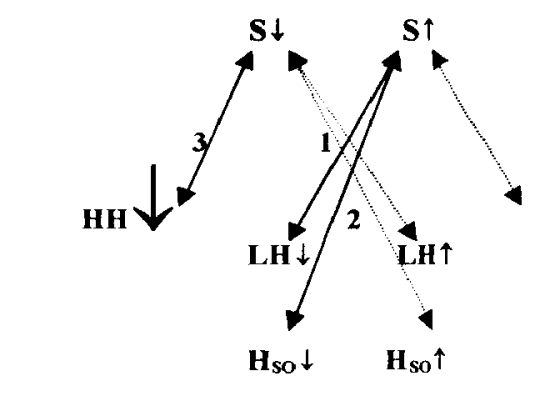

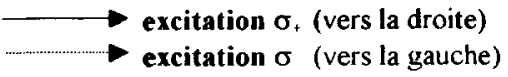

Schéma à deux particules
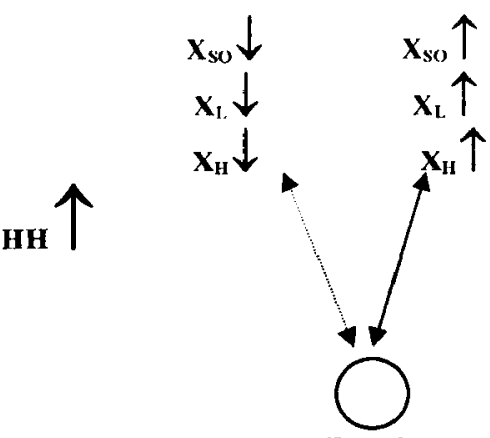

Etat de

base

\section{POLARISATION LINEAIRE}

Pas de Schéma à une particule

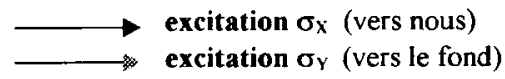

Schéma à deux particules

Insert expérimental :

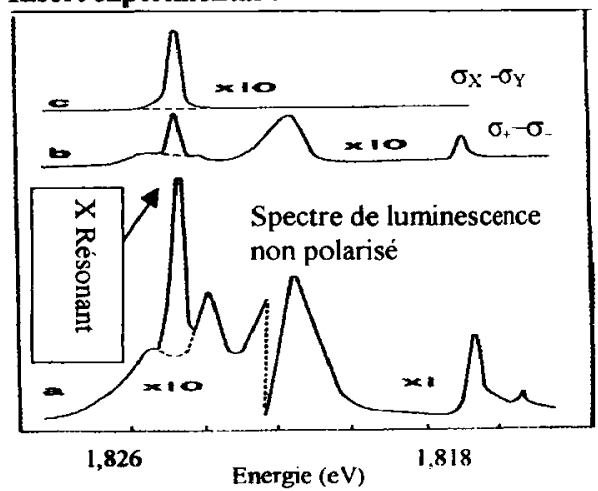

\section{Xso X}

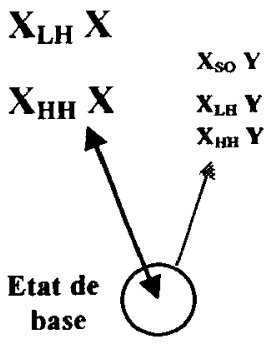

Figure 4.9 : Schémas des transitions possibles en polarisation à partir des bandes de trous lourds, des trous légers, et des trous SO. Dans un matériau cubique (Cf. fig. 2.2), les probabilités de transitions sont proportionnelles respectivement à 3,1 et 2 . Dans un hexagonal ou une hétérostructure, avec l'axe de symétrie parallèle à la propagation, c'est à peine plus compliqué. En tout cas, notez que $3=1+2 !$. On comprend la différence entre la base circulaire ( $\sigma^{+}$et $\sigma$ sont conjugués par renversement du temps) et la base linéaire $\left(\sigma^{x}\right.$ et $\sigma^{y}$ ne le sont pas). Dans l'insert expérimental, seule la création résonante d'excitons (ici par une cascade de phonons) conserve la polarisation linéaire. (D'après R. Planel [57]) 
- L'excitation préférentielle des bandes de valence légères, de moment angulaire $m= \pm 1 / 2$, donnera une polarisation négative. Après relaxation du trou, la luminescence présentera donc un taux de polarisation circulaire de signe opposé à l'excitation. Cette expérience simple est devenue un moyen d'identification des trous légers dans les systèmes d'hétérostructures encore mal maîtrisées.

\subsubsection{La création résonante d'excitons}

Naturellement, la création d'une paire électron -trou corrélée (sous forme d'exciton) n'est pas incompatible avec l'orientation de spin. En utilisant les notations de la figure 2.2, on pourra ainsi définir un exciton lourd: $\left|\sigma_{+}\right\rangle=\left|S \downarrow \otimes X_{+} \uparrow\right\rangle$, où le premier terme représente un électron de conduction $S$ de spin $\downarrow$, créé à partir d'un électron de valence $X_{-} \downarrow$ (car la transition ne retourne pas le spin), laissant un trou $X_{+} \uparrow$. Symétriquement, on pourra définir l'exciton $|\sigma-\rangle$, mettant en évidence une sorte d'isomorphisme entre l'espace des polarisations de photons et d'excitons. Il est donc légitime de définir par combinaison linéaire des excitons $\left|\sigma_{x}\right\rangle$ et $|\sigma y\rangle$, polarisés linéairement, et qui ne portent aucune orientation de spin particulière. On peut se convaincre [61] que ces états sont stabilisés parce que les états de type $\left|\sigma_{+}\right\rangle=\left|S \downarrow \otimes X_{+} \uparrow\right\rangle$ et $\left|S \uparrow \otimes X_{+} \uparrow\right\rangle$ n'ont pas la même énergie.

La raison en est assez subtile. Notons au passage que le deuxième état écrit plus haut est interdit optiquement, précisément parce que la lumière ne retourne pas le spin. En fait, à cause de la dégénérescence de spin, on peut construire $2 \times 2=4$ états ; deux sont permis optiquement (de moment total $J=1$ ) et deux sont interdits $(J=2)$. La levée de dégénérescence entre ces deux doublets est une réminiscence du fait que le trou est formé de $\mathrm{N}-1$ électrons, ce qui permet de parler paradoxalement d'interaction d'échange électron-trou. Sa valeur est proportionnée à la probabilité de présence de l'électron sur le trou, donc intimement liée au couplage avec la lumière. Elle varie, typiquement, de quelques $0,1 \mathrm{meV}$ dans les III-V, à un ordre de grandeur supérieure dans les II-VI.

Au plan expérimental, on comprend donc que la mise en évidence d'une mémoire de polarisation linéaire nécessite de traiter avec des excitons tout au long du processus de relaxation entre excitation et luminescence. C'est ce qu'illustrent les spectres de polarisation de la figure 4.9 .

\subsection{Transitions intrabandes}

Je reviens maintenant aux transitions optiques induites par le couplage A.p entre fonctions enveloppes. Comme on l'a vu plus haut, elles sont nécessairement intrabandes. Ce sujet, qui est né avec les hétérostructures, est encore en plein développement mais certaines applications, les détecteurs "infrarouge", sont déjà sur le marché. Plutôt qu'à un article de revue, on peut renvoyer à un compte rendu de conférence récent, qui lui est entièrement dédié [62]. Je discuterai plus avant en m'appuyant sur l'exemple des transitions à l'intérieur de la bande de conduction, les plus étudiées et utilisées jusqu'à présent.

Dans les puits quantiques, où donc la fonction enveloppe en $\mathrm{z}$ n'a pas la forme d'une onde plane, l'élément de matrice de $\mathrm{p}_{\mathrm{z}}$ (parallèle à l'axe de croissance) ne se réduit pas à une distribution de Dirac exprimant la conservation du moment. Ces transitions intrabandes 
"directes", c'est-à-dire non-assistées par phonons, n'ont pas d'équivalent dans les semiconducteurs massifs. L'élément de matrice de $\mathbf{p}$ est donc :

$$
\left\langle\mathrm{F}_{\mathrm{f}}|\mathbf{p}| \mathrm{F}_{\mathrm{i}}\right\rangle=\left\langle\boldsymbol{\varphi}_{\mathrm{f}}(\mathrm{z})\left|\mathrm{p}_{\mathrm{z}}\right| \varphi_{\mathrm{i}}(\mathrm{z})\right\rangle \delta\left(\mathbf{k}_{\perp}-\mathbf{q}\right)
$$

où subsiste une condition de conservation du $\mathbf{k}_{\perp}$ (dans le plan des couches). Comme les deux courbes de dispersion sont parallèles (aux effets de non parabolicité près), tous les états de différents $\mathbf{k}_{\perp}$ participent à la transition à la même énergie ( $C f$. fig. 4.10). On a donc une raie et non un bord d'absorption, comme dans le cas interbande.

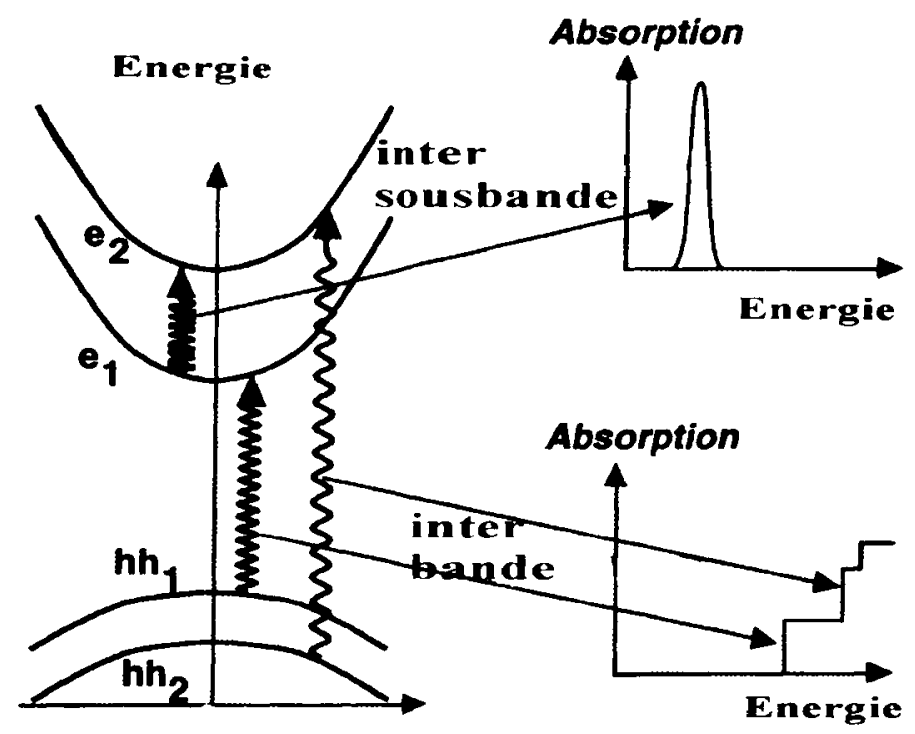

Figure 4.10 : Schéma des transitions interbandes et inter-sous-bandes (intrabandes) dans un puits quantique. (Emprunté à V. Berger[63])

D'autre part, l'élément en $\mathrm{p}_{\mathrm{z}}$ induit une transition si la lumière est polarisée le long de $\mathrm{z}$, ce qui exclut par exemple de travailler en incidence normale. II y est associé un ensemble de règles de sélection inverses de celles des transitions interbandes. Ainsi, dans un puits symétrique, seuls les états de parité différente seront couplés. Il va de soi aussi que l'absorption nécessite la présence d'électrons dans la bande de conduction, obtenus par dopage ou bien par illumination.

Les énergies typiques de ces transitions, compte tenu des possibilités technologiques et des masses effectives, se situent entre 0.01 et $1 \mathrm{eV}$, un intérêt particulier étant porté à la région du moyen infrarouge (avec la bande des $10 \mu \mathrm{m}$ ), où les principales possibilités étaient auparavant offertes par les transitions interbandes dans les alliages $\mathrm{HgCdTe}$.

Il est intéressant de discuter de l'intensité de ces transitions en termes de force d'oscillateur, exprimé plutôt par les éléments de matrice de $r$. En procédant par identification comme dans le cas des transitions atomiques ou moléculaires, on peut définir une force d'oscillateur par électron, associée à la transition entre les états i et $\mathrm{f}$ d'énergie $\mathrm{E}_{\mathrm{if}}$ :

$$
\mathrm{f}_{\text {if }}=\frac{2 \mathrm{~m}}{\hbar^{2}} \mathrm{E}_{\text {if }}\left|\left\langle\varphi_{\mathrm{f}}(\mathrm{z})\left|\mathrm{p}_{\mathrm{z}}\right| \varphi_{\mathrm{i}}(\mathrm{z})\right\rangle\right|^{2}
$$


où $\mathrm{m}$ est la masse effective, et non la masse de l'électron libre comme on l'introduit usuellement dans la force d'oscillateur. Or les fonctions enveloppes sont fonctions propres

a)

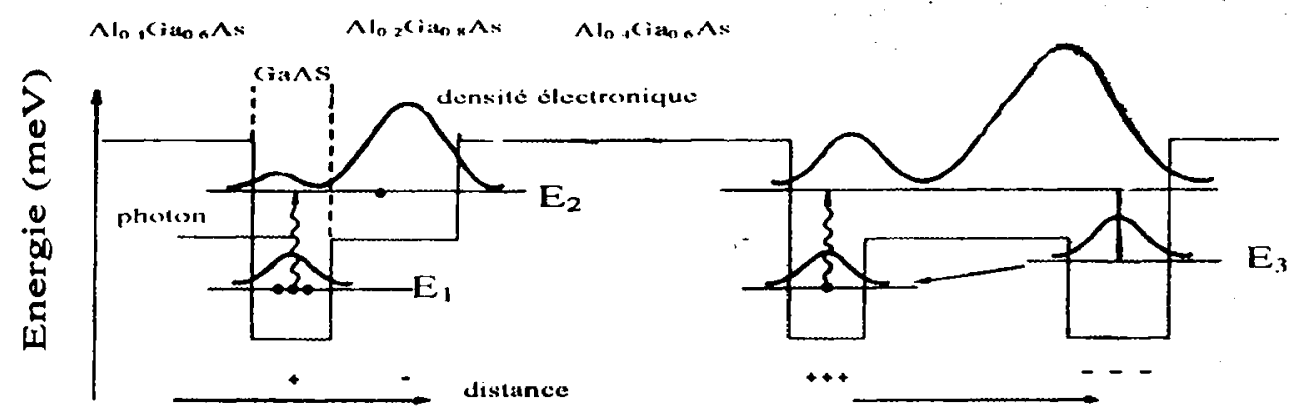

c)

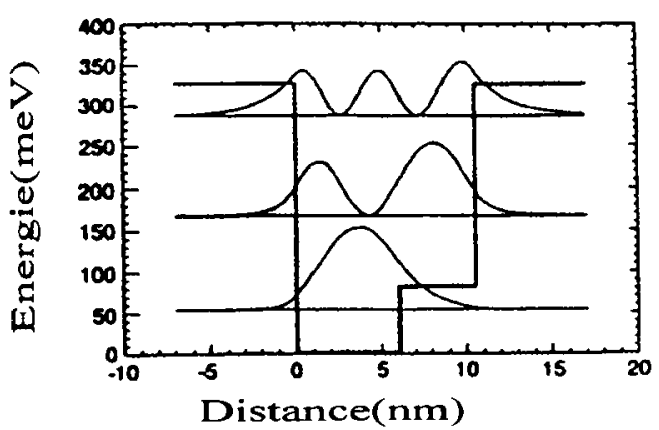

b)

d)

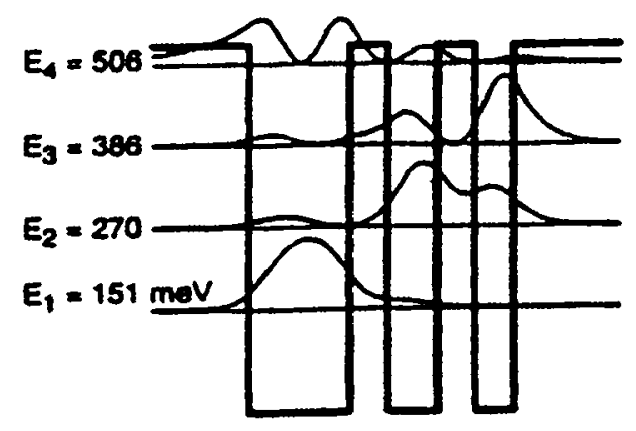

Figure 4.11 : Structures dessinées pour des transitions intrabandes induisant: a) la rectification optique. b) la rectification optique à meilleur rendement, mais à retour à l'équilibre plus lent. c) le doublement de fréquence résonant. d) le triplement de fréquence résonant. ( D'après V. Berger [63] et références citées)

d'un hamiltonien effectif $\mathrm{H}$ qui satisfait aux relations de commutation usuelles : $\left[\mathrm{z}, \mathrm{p}_{\mathrm{z}}\right]=\mathrm{i} \hbar$ et $[H, z]=-i \hbar p_{z} / m$. On peut donc [64] en déduire la règle de somme classique : $\sum_{n=2}^{\infty} f_{1 n}=1$, qui s'applique donc aux transitions intrabandes entre le niveau fondamental $n=1$ et les niveaux excités.

Cette relation valide la définition d'une force d'oscillateur "effective". Il est facile et instructif de la calculer pour la transition $1 \Rightarrow 2$ dans l'approximation du puits infini, de largeur L. Les fonctions enveloppes et les énergies des deux premiers états confinés sont :

$\mathrm{n}=1 \quad(2 / L)^{1 / 2} \cos \mathrm{z} \pi / \mathrm{L}$ d'énergie $\mathrm{h}^{2} \pi^{2} / 2 \mathrm{~mL}^{2}$

$\mathrm{n}=2 \quad(2 / \mathrm{L})^{1 / 2} \sin 2 \mathrm{z} \pi / \mathrm{L}$ d'énergie $4 \mathrm{~h}^{2} \pi^{2} / 2 \mathrm{~mL}^{2}$

Entre ces deux états, l'élément de matrice de l'opérateur $\mathrm{z}$ est : 


$$
\langle z\rangle=\langle n=1|z| n=2\rangle=2 / L \int_{-L / 2}^{+L / 2} \cos (z \pi / L) \sin (2 z \pi / L) z \delta z=16 L / 9 \pi^{2}
$$

qui est de l'ordre de grandeur de la largeur du puits, la dizaine de nm, et non, comme dans une molécule, le dixième de $\mathrm{nm}$. La force d'oscillateur est donc : $\mathrm{f}_{12}=2 \mathrm{mE}_{12}<\mathrm{z}>2 / \mathrm{h}^{2}=0,96$, valeur très proche du maximum, l'unité. Si l'on veut comparer ces structures aux systèmes atomiques et moléculaires, on peut noter que pour une énergie de transition donnée, le carré du dipôle sera ici beaucoup plus grand, en raison inverse de la masse effective.

Un puits quantique carré infini est donc très proche de l'oscillateur harmonique idéal où la force des transitions $1 \Rightarrow 3,4, \ldots$ est négligeable. Mais on voit combien ces forces d'oscillateur sont dépendantes de la forme précise du puits, comme d'ailleurs les énergies de transitions vers les divers états excités. On dispose là d'un nouvel outil pour l'ingénieur, qui permet de distribuer la force d'oscillateur au mieux de son intérêt, et, plus généralement, de dessiner les fonctions enveloppes les plus adéquates, par exemple pour optimiser des nonlinéarités. J'en donnerai deux exemples particulièrement représentatifs de la démarche.

4.9.1 Il est d'abord possible d'introduire volontairement de l'asymétrie dans le système, pour obtenir des non-linéarités d'ordre 2 . La figure $4.11 \mathrm{a}, \mathrm{b}$ représente des structures réalisées pour la rectification optique, c'est-à-dire l'apparition d'une tension continue sous éclairement. On ne peut mieux faire comprendre l'origine microscopique de ce genre d'effet qu'en imaginant ce qui se passe à la résonance, c'est-à-dire lors d'une transition intrabande réelle. Le centre de gravité de la charge est déplacé d'autant plus que l'asymétrie est violente. Mais, à l'inverse, la force d'oscillateur de la transition diminuera avec l'asymétrie, comme le recouvrement des probabilités de présence des deux états. Il y a donc un compromis à effectuer pour optimiser l'effet.

La structure b, qui comporte un second puits, permet d'accroître considérablement le temps de retour à l'équilibre, et donc la charge moyenne déplacée en régime permanent, au dépend de la rapidité du système. Là aussi, le meilleur compromis pour l'application visée est accessible à l'ingénieur.

4.9.2 Dans le cas de la génération de seconde harmonique, il est intéressant aussi d'optimiser les résonances (structure $4.11 \mathrm{c}$ ). Le choix d'un potentiel en créneau permet d'égaler les énergies des transitions $1 \Rightarrow 2$ et $2 \Rightarrow 3$. Le terme doublement résonant de la susceptibilité d'ordre 2 correspond donc à la succession des transitions $1 \Rightarrow 2 \Rightarrow 3 \Rightarrow 1$. L'interprétation pas à pas de cette succession, qui ignore les autres termes non résonants et lés interférences possibles avec eux, est la suivante : deux absorptions successives d'un photon, associées à deux promotions successives de l'électron puis suivies d'une relaxation radiative émettant un photon d'énergie double. Mais en tout cas, il est essentiel qu'aucune de ces transitions ne soit interdite, ce qui serait le cas pour $3 \Rightarrow 1$ dans une structure symétrique. La figure $4.11 \mathrm{c}, \mathrm{d}$ donne deux exemples d'optimisation de structures pour ce type d'application, et qui sont à la portée du "mécanicien quantique". 


\section{CONCLUSION}

J'espère avoir contribué dans ce cours à présenter au non-spécialiste l'essentiel de ce qu'il faut avoir compris pour utiliser au mieux les matériaux semi-conducteurs en optique. J'ai été volontairement discret sur ce que peuvent apporter les arguments de symétrie dans ces matériaux très généralement cristallins. Il me semble que cet aspect des choses, bien compris depuis les années 60, n'est pas spécifique aux semi-conducteurs. Par contre, avec de longs développements sur les excitons et les effets de confinement, j'ai insisté sur les expériences quasi résonantes, où donc une au moins des énergies optiques en jeu est proche du bord de bande interdite. C'est en effet dans cette direction, me semble-t-il, que peuvent apporter du nouveau à l'optique les derniers développements de la physique des semi-conducteurs, en particulier ceux rendus possibles par les formidables progrès de la technologie des micro- et nanostructures.

J'espère aussi avoir transmis, au-delà des développements mathématiques, une part importante de ma «culture » dans le domaine, qui s'exprime par des impressions qualitatives, des priorités dans l'exposé, sans doute des insuffisances. Elle s'est faite au contact de maitres et de collègues, principalement du Groupe de Physique des Solides de l'ENS, du laboratoire de Physique de la Matière Condensée de l'École Polytechnique, et du Groupement Scientifique CNET-CNRS de Bagneux. Ils sont nombreux, mais parmi les maîtres, je dois citer C. Benoit à la Guillaume et G. Lampel ; parmi les collègues, G. Bastard, F. Julien, F. Mollot, J.L. Oudar et C. Weisbuch.

Enfin, je tiens à remercier particulièrement ceux qui m'ont soutenu de près dans la rédaction de ce cours, par leur aide pratique, leurs encouragements et leurs critiques stimulantes. Ce sont G. Fishman, P. Sénellart et V. Thierry-Mieg.

\section{Références}

[1] Kittel Ch., Introduction to Solid State Physics, $7^{\text {th }}$ edn. (Wiley, New York, 1995).

[2] Ascroft N.W. et Mermin N.D, Solid State Physics (Holt-Saunders Int.Ed., Philadelphie 1976).

[3] Mathieu H., Physique des Semi-conducteurs et des Composants Electroniques (Masson, Paris, 1996).

[4] Haug H. et Koch S. Quantum theory of the Optical and Electronic properties of Semiconductors (World Scientific, Singapour, 1990).

[5] Klingshirn C.F., Semiconductor Optics (Springer-Verlag, Berlin Heidelberg, 1997).

[6]...Yu P. et Cardona M., Fundamental of Semiconductors, (Springer-Verlag, Berlin Heidelberg, 1996).

[7] Effets radiatifs dans les semi-conducteurs, éditeurs scientifiques J. Cernogora et R. Planel (Annales de Physique, 20C2, 1995).

[8] Zangwill A., Physics at Surfaces (Cambridge University Press, Cambridge).

[9] Les lasers et leurs applications scientifiques et médicales, éditeurs scientifiques C. Fabre et J.P. Pocholle, Collection de la Société Française d'Optique, (EDP Sciences, Paris, 1996).

[10] Weisbuch Cl., Physique des lasers à semi-conducteurs in réf.7, p.353.

[11] Cf. par exemple réf. 1 p.432 ou réf. 2 p. 655.

[12] Gaj J. thèse d'habilitation, Université de Varsovie (1981). 
[13] Bastard G., Wave mechanics applied to Semiconductor Heterostructures (Editions de Physique, Paris, 1988).

[14] Heterojunctions and Semiconductor Superlattices, Editors G. Allan, G. Bastard, N. Boccara, M. Lannoo et M. Voos (Springer-Verlag, Berlin Heidelberg, 1986).

[15] Weisbuch C., Miller R.C., Dingle R., Gossard A.C. et Wiegmann W., Sol. St. Comm. 37(1981) 219.

[16] Goldstein L., Horikoshi Y., Tarucha S. et Okamoto H., Jpn.J.Appl.Phys.22 (1983) 1489 ; Deveaud B., Emery J.Y., Chomette A., Lambert B. et Baudet M., Appl.Phys. Lett. 45 (1984) 1078.

[17] Paquet D., Superlatt. and Microstr. 2 (1986) 429.

[18] Jusserand B., Mollot F., Moison J.M. et Le Roux G., Appl. Phys. Lett. 57 (1990) 560.

[19] Gammon D., Shanabrook B.V et Katzer D.S., Appl. Phys. Lett. 57(1990) 2710 ; Phys Rev. Lett. 67 (1991) 1547.

[20] Gammon D., Snow E.S. et Katzer D.S., Appl. Phys. Lett. 67 (1995) 2391.

[21] Altarelli M., Band Structures, Impurities and Excitons in Superlattices, dans réf.14, p.16.

[22] Marzin J.Y., Strained Superlattices dans réf.14, p.161.

[24] Gershoni D., Weiner J.S., Chu S.N.G., Baraf G.A., Vandenberg J.M., Pfeiffer L.N., West K., Logan R.A. et Tanbun-Ek T., Phys. Rev. Lett. 65, (1990) 1631 Phys. Rev. Lett. 71 (1993) 4071.

[25] Etienne B., Laruelle F., Bloch J., Sfaxi L. et Lelarge F., J.Cryst. Growth 150 (1995) 336.

[26] Goldstein L., Glas F., Marzin J.Y., Charasse M.N. et Le Roux G., Appl.Phys.Lett. 47 (1985) 1099 ; pour une revue récente, cf. Gérard J.M. dans réf.27 p.357.

[27] Confined Electrons and Photons, New Physics and Applications, éd. E. Burstein et Cl. Weisbuch (Plenum Press, New York, 1995).

[28] Laruelle F. Thèse, Université Paris 7, (1988) ; Pern-Fei Yuh et Wand K.L., Phys. Rev. B38 (1988) 13307.

[29] Kane E.O. J.Phys. Chem. Solids 1 (1957) 249.

[30] Luttinger J.M., Phys. Rev. 102 (1956) 1030.

[31] Donner de telles listes de paramètres est toujours un casse-tête. Celle-ci, assez ancienne, se trouve dans YC. p.165, où des réserves sont émises. De façon générale, il faut connaître l'existence des Tables de Landoldt et Börnstein, (Springer-Verlag, Berlin Heidelberg) tout en gardant si possible un certain sens critique.

[32] Butcher P.N. et Cotter D., The elements of Non Linear Optics (Cambridge University Press, 1990) p. 109.

[33] Charfi F. Thèse, Faculté des Sciences de Tunis (1984); Phys. Rev B33 (1986) 5623.

[34] Zhakharchenya B.P., Mirlin D.N., Perel V.I. et Reshina I.I., Sov. Phys. Solid State 20 (1978) 1250 ; Sov.Phys. Usp. 25 (1982) 143.

[35] Knox R.S., Theory of Excitons, Solid State Physics, Suppl.5 (Academic, New York, 1965).

[36] Excitons éd. E.I. Rashba et M.D. Sturge (North Holland, Amsterdam, 1982).

[37] Combescot M., On Wannier Excitons, dans réf.7 p.395.

[38] Baldereschi A. et Lipari N.O., Phys. Rev. B3 (1971) 439 ; Altarelli M. and Lipari N.O., Phys. Rev. B15 (1977) 4898.

[39] par exemple Haug $H$. et Koch S., dans réf.4, appendice A.

[40] Ce calcul est présenté pour le cas assez restrictif $m_{e}=m_{h}$ dans : Haug H. et Koch S., dans réf.4, p.211. 
[41] Il n'y a pas de meilleure illustration expérimentale de la délocalisation de l'exciton que la transmission du polariton-excitonique, par exemple : Itoh T., Lavallard Ph., Reydellet J., et Benoit à la Guillaume B., Sol.St. Comm. 37 (1981).

[42] Ce sujet "garde une part de mystère ", comme l'avoue récemment $\mathrm{Ph}$. Nozières dans : Some Comments on the Mott Transition in Metals and Semiconductors, dans réf.7, p 417.

[43] Ma présentation doit beaucoup à celle de C.F. Klingshirn dans réf.5 chap.20., qui renvoie à de nombreuses références. Une présentation théorique plus autonome se trouve dans Haug H. et Koch S., dans réf. 4 chap. 8.

[44] par exemple par C.F. Klingshirn dans réf.5, p.259 pour le QCSE, p.366 pour l'effet Wannier Stark et l'oscillateur de Bloch.

[45] Miller D.A.B., Chemla D.S., Damen T.C., Koren U., Gossard A.C., Wiegmann W., Wood T.H. et Burrus C.A., Phys.Rev B32 (1985) 1043 ; Phys.Rev.B33 (1986) 6976.

[46] Bleuse J., Bastard G. et Voisin P., Phys.Rev.Lett. 60 (1988) 220.

[47] Le premier article d'Ezaki et Tsu (IBM J.Res.Develop. 14 (1970) 61) suggérant l'observation des Oscillations de Bloch dans des microstructures artificielles a été refusé à la Physical Review parce que «trop spéculatif », en 1969 ; à défaut, on pourra voir Sibille A., Palmier J.F. et Mollot F, Appl.Phys;Lett. 60 (1990) 457 ; pour une revue plus récente cf. Palmier J.F., Minot Ch., Harmand J.C., Sibille A., Tanguy D. et Penard E., à paraître dans Superlatt. and Microstr., 1999.

[48] par exemple Bastard G., Mendez E.E., Chang L.L. et Ezaki L., Phys.Rev. B26 (1982) 1974.

[49] Lefèbvre P., Christol Ph. et Mathieu H., Phys.Rev. B48 (1993) 17308.

[50] Elliott R.J., Phys.Rev.108 (1957) 1384.

[51] Hopfield J.J. Phys.Rev. 112 (1958) 1555 ; Proc.Int. Conf. Phys. Semicond. (Kyoto,1966) p.77. Ce dernier article a été reproduit et replacé dans le contexte d'aujourd'hui dans la référence 27.

[52] Cf. réf. 1 p. 150 ; réf. 2 p.551 ; réf.5 chap.5.

[53] Pour une revue sur les ABC's, cf. Ivchenko E.L. dans réf. 36 chap.4.

[54] Houdré R., Stanley R.P., Oesterle U., Ilegems M. et Weisbuch C., Phys.Rev.B49 (1994) 16761.

[55] Fainstein A., Jusserand B. et Thierry-Mieg V., Phys. Rev. B53 (1996) R13287.

[56] Lovering D.J., Fino G. Simmonneau C. Kuszelewicz R. Azoulay R. Levenson J.A. Electr. Lett. 32 (1996) 1782.

[57] Planel R., Sol. State Electr. 21 (1978) 1437.

[58] Optical Orientation, ed. Meyer et Zhakharchenya B.P. (North Holland, Amsterdam, 1983).

[59] Pikus G.E. et Ivchenko E.I., Optical Orientation and Polarized Luminescence of Excitons in Semiconductors dans réf. 36, chap.6.

[60] Weisbuch C., communication privée.

[61] Planel R. et Benoit à la Guillaume C, Optical Orientation of Exciton, in réf.58 chap.8. Pour une expérience récente et lumineuse mettant en évidence le rôle de l'interaction d'échange électron-trou, cf. Amand Th., Dareys B., Baylac B., Marie X., Barrau J., Brousseau M., Dunstan D.J. et Planel R., Phys Rev. B50 (1994) 11624

[62] Intersubband Transitions in Quantum Wells, Ed.S.Li et Y.K.Su, (Kluwer Academic Publishers, Boston, 1998). 
[63] Berger V., Frequency Conversion with semiconductor heterostructures, in Advanced Photonics with second-order Optical Non Linear processes, éd. A.Boardman, (NATO proceedings, Kluwer Academic Publishers, Boston,1999).

[64] Cette discussion est inspirée par: Rosencher E. et Bois Ph., Phys. Rev. B44 (1991) 11315. 